Supplement of Earth Syst. Sci. Data, 13, 4485-4527, 2021

https://doi.org/10.5194/essd-13-4485-2021-supplement

(c) Author(s) 2021. CC BY 4.0 License.

(c) (i)

Supplement of

\title{
Last Interglacial sea-level proxies in the western Mediterranean
}

\section{Ciro Cerrone et al.}

Correspondence to: Ciro Cerrone (ciro.cerrone@dst.unipi.it)

The copyright of individual parts of the supplement might differ from the article licence. 


\section{Supplementary Table}

Summary of sea-level datapoints and dated samples / chronostratigraphc constraints for the Western Mediterranean. Acronyms: Type of datapoints: SLI = Sea Level Index point; $T L I=$ Terrestrial Limiting; $M L I=$ Marine Limiting. RSL Indicator: $M T=$ Marine Terrace; $B R K=$ Beach deposit or beachrock; IMF = Shallow or intertidal marine fauna; LIT = Upper limit of Lithophaga boreholes; $L A G=$ Lagoonal deposit; $B S D=$ Beach swash deposit; $C O R=$ Single Coral; NTC = Tidal notch; FOR = Foreshore deposits; BIO = Bioerosional and erosional markers on limestone cliff; BRI = Beach Ridge; $A R I=$ Lithophyllum byssoides algal rims. In the age field, * denotes ages that were rejected by the original authors; > denotes that the age is "Older than", while < denotes that the age is "Younger than".

\begin{tabular}{|c|c|c|c|c|c|c|c|c|c|c|}
\hline $\begin{array}{l}\text { WALIS } \\
\text { ID }\end{array}$ & $\begin{array}{l}\text { Latitude } \\
\text { Longitude }\end{array}$ & Site & $\begin{array}{l}\text { Type of } \\
\text { datapoint }\end{array}$ & $\begin{array}{l}\text { RSL } \\
\text { Indicator }\end{array}$ & $\begin{array}{l}\text { Elevation } \\
\text { (m) }\end{array}$ & $\begin{array}{l}\text { Paleo RSL } \\
\text { (m) }\end{array}$ & Dating (Original ID) & $\begin{array}{l}\text { Age (ka or } \\
\text { MIS) }\end{array}$ & $\begin{array}{l}\text { Quality } \\
\text { (RSL, } \\
\text { Age) } \\
\end{array}$ & Reference(s) \\
\hline \multicolumn{11}{|c|}{ Spain (Andalucía) } \\
\hline 765 & $\begin{array}{l}36.3313 \\
-6.1589 \\
\end{array}$ & Torre del Puerco & SLI & MT & $8.7 \pm 1.7$ & $8.1 \pm 2.6$ & $\begin{array}{l}\text { STRAT (MIS 5e in the } \\
\text { Gibraltar Strait Area) }\end{array}$ & MIS 5e & 1,3 & Zazo et al., 1999 \\
\hline 764 & $\begin{array}{l}36.2251 \\
-6.0618 \\
\end{array}$ & Conil-Trafalgar & SLI & MT & $13 \pm 2.6$ & $12.4 \pm 3.3$ & $\begin{array}{l}\text { STRAT (MIS 5e in the } \\
\text { Gibraltar Strait Area) }\end{array}$ & MIS 5e & 1,3 & Zazo et al., 1999 \\
\hline 763 & $\begin{array}{c}36.1703 \\
-5.8894\end{array}$ & Zahara & SLI & MT & $14 \pm 2.8$ & $13.4 \pm 3.4$ & $\begin{array}{l}\text { STRAT (MIS 5e in the } \\
\text { Gibraltar Strait Area) }\end{array}$ & MIS 5e & 1,3 & Zazo et al., 1999 \\
\hline 762 & $\begin{array}{l}36.0841 \\
-5.8022 \\
\end{array}$ & Cabo gracia & SLI & MT & $13 \pm 2.6$ & $12.5 \pm 3.2$ & $\begin{array}{l}\text { STRAT (MIS 5e in the } \\
\text { Gibraltar Strait Area) }\end{array}$ & MIS 5e & 1,3 & Zazo et al., 1999 \\
\hline 761 & $\begin{array}{l}36.0751 \\
-5.7519 \\
\end{array}$ & Bolonia - Punta Paloma & SLI & MT & $13.5 \pm 2.7$ & $13 \pm 3.3$ & $\begin{array}{l}\text { STRAT (MIS 5e in the } \\
\text { Gibraltar Strait Area) }\end{array}$ & MIS 5e & 1,3 & Zazo et al., 1999 \\
\hline 760 & $\begin{array}{l}36.023 \\
-5.5687\end{array}$ & Tarifa & SLI & MT & $19 \pm 3.8$ & $18.9 \pm 3.9$ & $\begin{array}{l}\text { STRAT (MIS 5e in the } \\
\text { Gibraltar Strait Area) }\end{array}$ & MIS 5e & 1,3 & Zazo et al., 1999 \\
\hline 759 & $\begin{array}{l}36.0805 \\
-5.4313 \\
\end{array}$ & Algeciras 2 & SLI & MT & $13 \pm 2.6$ & $12.9 \pm 2.8$ & $\begin{array}{l}\text { STRAT (MIS 5e in the } \\
\text { Gibraltar Strait Area) }\end{array}$ & MIS 5e & 1,3 & Zazo et al., 1999 \\
\hline 758 & $\begin{array}{l}36.0958 \\
-5.4591\end{array}$ & Algeciras 1 & SLI & MT & $10 \pm 2$ & $9.9 \pm 2.2$ & $\begin{array}{l}\text { STRAT (MIS 5e in the } \\
\text { Gibraltar Strait Area) }\end{array}$ & MIS 5e & 1,3 & Zazo et al., 1999 \\
\hline 757 & $\begin{array}{l}36.1057 \\
-5.3352 \\
\end{array}$ & Gibraltar & SLI & MT & $5.3 \pm 1.1$ & $5.1 \pm 1.4$ & $\begin{array}{l}\text { STRAT (MIS 5e in the } \\
\text { Gibraltar Strait Area) }\end{array}$ & MIS 5e & 1,3 & Zazo et al., 1999 \\
\hline 756 & $\begin{array}{l}36.1865 \\
-5.3379 \\
\end{array}$ & La Linea & SLI & MT & $6.5 \pm 1.3$ & $6.4 \pm 1.6$ & $\begin{array}{l}\text { STRAT (MIS 5e in the } \\
\text { Gibraltar Strait Area) }\end{array}$ & MIS 5e & 1,3 & Zazo et al., 1999 \\
\hline 755 & $\begin{array}{l}36.5071 \\
-4.8502\end{array}$ & Marbella & SLI & MT & $2 \pm 0.4$ & $1.9 \pm 1$ & $\begin{array}{l}\text { STRAT (MIS 5e in the } \\
\text { Gibraltar Strait Area) }\end{array}$ & MIS 5e & 1,3 & Zazo et al., 1999 \\
\hline 754 & $\begin{array}{l}36.5278 \\
-4.6292\end{array}$ & Fuengirola & SLI & MT & $2 \pm 0.4$ & $1.9 \pm 1$ & $\begin{array}{l}\text { STRAT (MIS 5e in the } \\
\text { Gibraltar Strait Area) }\end{array}$ & MIS 5e & 1,3 & Zazo et al., 1999 \\
\hline 753 & $\begin{array}{l}36.6104 \\
-4.4837 \\
\end{array}$ & Torremolinos & SLI & MT & $2 \pm 0.4$ & $1.9 \pm 1$ & $\begin{array}{l}\text { STRAT (MIS 5e in the } \\
\text { Gibraltar Strait Area) }\end{array}$ & MIS 5e & 1,3 & Zazo et al., 1999 \\
\hline 752 & $\begin{array}{l}36.7155 \\
-4.3176 \\
\end{array}$ & El Candado & SLI & MT & $1.5 \pm 0.3$ & $1.4 \pm 1$ & $\begin{array}{l}\text { STRAT (MIS 5e in the } \\
\text { Gibraltar Strait Area) }\end{array}$ & MIS 5e & 1,3 & Zazo et al., 1999 \\
\hline 4001 & $\begin{array}{l}36.713164 \\
-4.338721\end{array}$ & Cerro Juan & SLI & BRK & $2 \pm 0.9$ & $2.1 \pm 1.1$ & ESR (E-92) & $117.3 \pm 23.4$ & 2,3 & Brückner and Radtke (1986) \\
\hline \multicolumn{11}{|c|}{ Spain (Murcia) } \\
\hline 750 & $\begin{array}{l}37.45904 \\
-1.47611\end{array}$ & $\begin{array}{l}\text { Cope Basin - Point } 11 \\
\text { Unit D }\end{array}$ & SLI & BRK & $3.8 \pm 1.6$ & $4 \pm 1.7$ & $\begin{array}{l}\text { STRAT (Unit D - Cope } \\
\text { Basin MIS 5e) }\end{array}$ & MIS 5 & 1,3 & $\begin{array}{l}\text { Bardají et al., } 2015 \\
\text { Zazo et al., } 2003\end{array}$ \\
\hline
\end{tabular}




\begin{tabular}{|c|c|c|c|c|c|c|c|c|c|c|}
\hline $\begin{array}{l}\text { WALIS } \\
\text { ID }\end{array}$ & $\begin{array}{l}\text { Latitude } \\
\text { Longitude }\end{array}$ & Site & $\begin{array}{l}\text { Type of } \\
\text { datapoint }\end{array}$ & $\begin{array}{l}\text { RSL } \\
\text { Indicator }\end{array}$ & $\begin{array}{l}\text { Elevation } \\
\text { (m) }\end{array}$ & $\begin{array}{l}\text { Paleo RSL } \\
\text { (m) }\end{array}$ & Dating (Original ID) & $\begin{array}{l}\text { Age (ka or } \\
\text { MIS) }\end{array}$ & $\begin{array}{l}\text { Quality } \\
\text { (RSL, } \\
\text { Age) }\end{array}$ & Reference(s) \\
\hline 749 & $\begin{array}{l}37.46034 \\
-1.47571\end{array}$ & $\begin{array}{l}\text { Cope Basin - Point } 10 \\
\text { Unit D }\end{array}$ & SLI & BRK & $3.5 \pm 2.2$ & $3.7 \pm 2.3$ & $\begin{array}{l}\text { STRAT (Unit D - Cope } \\
\text { Basin MIS 5e) }\end{array}$ & MIS 5 & 1,3 & $\begin{array}{l}\text { Bardají et al., } 2015 \\
\text { Zazo et al., } 2003\end{array}$ \\
\hline 748 & $\begin{array}{l}37.46175 \\
-1.47514\end{array}$ & $\begin{array}{l}\text { Cope Basin - Point } 9 \\
\text { Unit D }\end{array}$ & SLI & BRK & $3.8 \pm 1.3$ & $4 \pm 1.4$ & $\begin{array}{l}\text { STRAT (Unit D - Cope } \\
\text { Basin MIS 5e) }\end{array}$ & MIS 5 & 1,3 & $\begin{array}{l}\text { Bardají et al., } 2015 \\
\text { Zazo et al., } 2003\end{array}$ \\
\hline 747 & $\begin{array}{l}37.46336 \\
-1.47436\end{array}$ & $\begin{array}{l}\text { Cope Basin - Point } 8 \\
\text { Unit D }\end{array}$ & SLI & BRK & $2.8 \pm 1.3$ & $3 \pm 1.4$ & $\begin{array}{l}\text { STRAT (Unit D - Cope } \\
\text { Basin MIS 5e) }\end{array}$ & MIS 5 & 1,3 & $\begin{array}{l}\text { Bardají et al., } 2015 \\
\text { Zazo et al., } 2003\end{array}$ \\
\hline 746 & $\begin{array}{l}37.46472 \\
-1.47312 \\
\end{array}$ & $\begin{array}{l}\text { Cope Basin - Point } 7 \\
\text { Unit D }\end{array}$ & SLI & BRK & $4.5 \pm 1.1$ & $4.7 \pm 1.3$ & $\begin{array}{l}\text { STRAT (Unit D - Cope } \\
\text { Basin MIS 5e) }\end{array}$ & MIS 5 & 1,3 & $\begin{array}{l}\text { Bardají et al., } 2015 \\
\text { Zazo et al., } 2003\end{array}$ \\
\hline 745 & $\begin{array}{l}37.46731 \\
-1.47165\end{array}$ & $\begin{array}{l}\text { Cope Basin - Point } 6 \\
\text { Unit D }\end{array}$ & SLI & BRK & $3 \pm 1.4$ & $3.2 \pm 1.6$ & $\begin{array}{l}\text { STRAT (Unit D - Cope } \\
\text { Basin MIS 5e) }\end{array}$ & MIS 5 & 1,3 & $\begin{array}{l}\text { Bardají et al., } 2015 \\
\text { Zazo et al., } 2003\end{array}$ \\
\hline 744 & $\begin{array}{l}37.47374 \\
-1.46843\end{array}$ & $\begin{array}{l}\text { Cope Basin - Point } 5 \\
\text { Unit D }\end{array}$ & SLI & BRK & $4.3 \pm 1.3$ & $4.5 \pm 1.4$ & $\begin{array}{l}\text { STRAT (Unit D - Cope } \\
\text { Basin MIS 5e) }\end{array}$ & MIS 5 & 1,3 & $\begin{array}{l}\text { Bardají et al., } 2015 \\
\text { Zazo et al., } 2003\end{array}$ \\
\hline 743 & $\begin{array}{l}37.47298 \\
-1.46863 \\
\end{array}$ & $\begin{array}{l}\text { Cope Basin - Point } 4 \\
\text { Unit D }\end{array}$ & SLI & BRK & $2.9 \pm 1.5$ & $3.1 \pm 1.6$ & $\begin{array}{l}\text { STRAT (Unit D - Cope } \\
\text { Basin MIS 5e) }\end{array}$ & MIS 5 & 1,3 & $\begin{array}{l}\text { Bardají et al., } 2015 \\
\text { Zazo et al., } 2003\end{array}$ \\
\hline 742 & $\begin{array}{l}37.47436 \\
-1.46725 \\
\end{array}$ & $\begin{array}{l}\text { Cope Basin - Point } 3 \\
\text { Unit D }\end{array}$ & SLI & BRK & $7.5 \pm 1.1$ & $7.7 \pm 1.3$ & $\begin{array}{l}\text { STRAT (Unit D - Cope } \\
\text { Basin MIS 5e) }\end{array}$ & MIS 5 & 1,3 & $\begin{array}{l}\text { Bardají et al., } 2015 \\
\text { Zazo et al., } 2003\end{array}$ \\
\hline 741 & $\begin{array}{l}37.47534 \\
-1.46762\end{array}$ & $\begin{array}{l}\text { Cope Basin - Point } 2 \\
\text { Unit D }\end{array}$ & SLI & BRK & $8.5 \pm 1.1$ & $8.7 \pm 1.3$ & $\begin{array}{l}\text { STRAT (Unit D - Cope } \\
\text { Basin MIS 5e) }\end{array}$ & MIS 5 & 1,3 & $\begin{array}{l}\text { Bardají et al., } 2015 \\
\text { Zazo et al., } 2003\end{array}$ \\
\hline 740 & $\begin{array}{l}37.47626 \\
-1.46713 \\
\end{array}$ & $\begin{array}{l}\text { Cope Basin - Point } 1 \\
\text { Unit D }\end{array}$ & SLI & BRK & $7.5 \pm 1.1$ & $7.7 \pm 1.3$ & $\begin{array}{l}\text { STRAT (Unit D - Cope } \\
\text { Basin MIS 5e) }\end{array}$ & MIS 5 & 1,3 & $\begin{array}{l}\text { Bardají et al., } 2015 \\
\text { Zazo et al., } 2003\end{array}$ \\
\hline \multicolumn{11}{|c|}{ Spain (Comunidad Valenciana) } \\
\hline \multirow{2}{*}{751} & \multirow{2}{*}{$\begin{array}{l}38.159262 \\
-0.635852\end{array}$} & \multirow{2}{*}{$\begin{array}{l}\text { La Marina - El Pinet } \\
\text { Section A, Terrace T8 }\end{array}$} & \multirow{2}{*}{ SLI } & \multirow{2}{*}{ BRK } & \multirow{2}{*}{$5.5 \pm 0.3$} & \multirow{2}{*}{$5.7 \pm 0.7$} & U-series (MP02-7) & MIS 5 & \multirow{2}{*}{5,3} & \multirow{2}{*}{$\begin{array}{l}\text { Goy et al., } 2006 \\
\text { Mauz et al., } 2012\end{array}$} \\
\hline & & & & & & & LUM (LV382) & $114 \pm 15$ & & \\
\hline 738 & $\begin{array}{l}38.159262 \\
-0.635852 \\
\end{array}$ & $\begin{array}{l}\text { La Marina - El Pinet } \\
\text { Section B, Terrace T8 }\end{array}$ & SLI & BRK & $8 \pm 0.3$ & $8.2 \pm 0.7$ & U-series (MP02-6) & MIS 5e & 5,3 & $\begin{array}{l}\text { Goy et al., } 2006 \\
\text { Mauz et al., } 2012 \\
\end{array}$ \\
\hline \multirow{2}{*}{4079} & \multirow{2}{*}{$\begin{array}{l}38.200564 \\
-0.511227\end{array}$} & \multirow{2}{*}{$\begin{array}{l}\text { South of Torre de } \\
\text { Enmedio }\end{array}$} & \multirow{2}{*}{ SLI } & \multirow{2}{*}{ BRK } & \multirow{2}{*}{$1.5 \pm 0.3$} & \multirow{2}{*}{$1.7 \pm 0.7$} & U-series (E-32(b) & MIS 5 & \multirow{2}{*}{1,3} & \multirow{2}{*}{ Brückner, 1986} \\
\hline & & & & & & & ESR (E-32-b) & $94 \pm 18.8$ & & \\
\hline \multirow{2}{*}{4080} & \multirow{2}{*}{$\begin{array}{l}38.234985 \\
-0.513647\end{array}$} & \multirow{2}{*}{ Calabasi } & \multirow{2}{*}{ SLI } & \multirow{2}{*}{ BRK } & \multirow{2}{*}{$3 \pm 0.6$} & \multirow{2}{*}{$3.2 \pm 0.9$} & ESR (E-75-a) & $125 \pm 37.5$ & \multirow{2}{*}{1,3} & \multirow{2}{*}{ Brückner, 1986} \\
\hline & & & & & & & $\operatorname{ESR}(\mathrm{E}-75-b)$ & $89 \pm 17.8$ & & \\
\hline 4081 & $\begin{array}{l}38.352734 \\
-0.409682 \\
\end{array}$ & Cabo de las Huertas & SLI & BRK & $2 \pm 0.4$ & $2.2 \pm 0.8$ & ESR (E-70) & $112 \pm 22.4$ & 1,3 & Brückner, 1986 \\
\hline \multicolumn{11}{|c|}{ Spain (Cataloña) - Figure 9} \\
\hline \multirow{2}{*}{4002} & 40.839438 & $M$ & CII & DDY & $12=06$ & 1907 & ESR (E-63a) & $105 \pm 21$ & 20 & 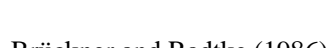 \\
\hline & 0.746244 & Morro del Gos & SLI & BRK & $1.3 \pm 0.6$ & $1.4 \pm 0.7$ & ESR (E-63b) & $137 \pm 27.4$ & 2,2 & Bruckner and Radtke (1986) \\
\hline Ane? & 41.059166 & Cabo de Salou & CII & PPY & $3+06$ & $32+0$ e & ESR (E-44(a) & $94 \pm 18.8$ & 13 & Drïckner 1086 \\
\hline 4082 & 1.16182 & Cala Font & SL1 & $\mathrm{BRK}$ & $3 \pm 0.0$ & $3.2 \pm 0.8$ & ESR (E-44(b) & $142 \pm 28.4$ & 1,3 & Bruckner, 1980 \\
\hline 1002 & 40.958015 & $C$ & CII & DOV & 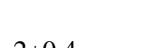 & 2007 & U-series (E-57-A(a) & MIS 5c & & $\mathrm{D}$ \\
\hline 4083 & 0.879556 & Cala de Gestell & SLI & BRK & $2 \pm 0.4$ & $2.2 \pm 0.1$ & ESR (E-57-A(a) & $99 \pm 29.7$ & 1,3 & Bruckner, 1986 \\
\hline
\end{tabular}




\begin{tabular}{|c|c|c|c|c|c|c|c|c|c|c|}
\hline $\begin{array}{l}\text { WALIS } \\
\text { ID }\end{array}$ & $\begin{array}{l}\text { Latitude } \\
\text { Longitude }\end{array}$ & Site & $\begin{array}{l}\text { Type of } \\
\text { datapoint }\end{array}$ & $\begin{array}{l}\text { RSL } \\
\text { Indicator }\end{array}$ & $\begin{array}{l}\text { Elevation } \\
\text { (m) }\end{array}$ & $\begin{array}{l}\text { Paleo RSL } \\
\text { (m) }\end{array}$ & Dating (Original ID) & $\begin{array}{l}\text { Age (ka or } \\
\text { MIS) }\end{array}$ & $\begin{array}{l}\text { Quality } \\
\text { (RSL, } \\
\text { Age) }\end{array}$ & Reference(s) \\
\hline & & & & & & & ESR (E-57-A(b) & $142 \pm 42.6$ & & \\
\hline \multirow{2}{*}{4084} & \multirow{2}{*}{$\begin{array}{l}40.899511 \\
0.822442\end{array}$} & \multirow{2}{*}{ Cala del Torrent del Pi } & \multirow{2}{*}{ SLI } & \multirow{2}{*}{ BRK } & \multirow{2}{*}{$1 \pm 0.2$} & \multirow{2}{*}{$1.2 \pm 0.6$} & U-series (E-58(a) & MIS 5 & \multirow{2}{*}{1,3} & \multirow{2}{*}{ Brückner, 1986} \\
\hline & & & & & & & ESR (E-58(a) & $142 \pm 42.6$ & & \\
\hline \multicolumn{11}{|c|}{ Spain (Islas Baleares, Mallorca) } \\
\hline 779 & $\begin{array}{l}39.543536 \\
2.700453\end{array}$ & Cala Pudent & SLI & BRK & $2.3 \pm 0.1$ & $2.9 \pm 1.1$ & $\begin{array}{l}\text { STRAT ( } P . \text { latus or } \\
\text { Senegalese fauna) }\end{array}$ & MIS 5 & 4,3 & $\begin{array}{l}\text { Lorscheid et al., } 2017 \\
\text { Butzer and Cuerda, } 1962 \\
\text { Cuerda, } 1979 \\
\text { Hearty, 1987b } \\
\text { Hillaire-Marcel et al., } 1996\end{array}$ \\
\hline \multirow{7}{*}{357} & \multirow{7}{*}{$\begin{array}{l}39.542175 \\
2.702049\end{array}$} & \multirow{7}{*}{$\begin{array}{l}\text { Camp de Tir } \\
\text { Unit U4 }\end{array}$} & \multirow{7}{*}{ SLI } & \multirow{7}{*}{ IMF } & \multirow{7}{*}{$1.3 \pm 0$} & \multirow{7}{*}{$2.1 \pm 0.7$} & U-series (MA-1) & $119.2 \pm 1.7$ & \multirow{7}{*}{5,4} & \multirow{7}{*}{$\begin{array}{l}\text { Muhs et al., } 2015 \\
\text { Butzer and Cuerda, } 1962 \\
\text { Hearty, 1987b } \\
\text { Hillaire-Marcel et al., } 1996 \\
\text { Lorscheid et al., } 2017\end{array}$} \\
\hline & & & & & & & U-series (MA-4) & $118.1 \pm 1.3$ & & \\
\hline & & & & & & & U-series (MA-5) & $114.8 \pm 2$ & & \\
\hline & & & & & & & U-series (MA-6) & $119.4 \pm 1.6$ & & \\
\hline & & & & & & & U-series (MA-7) & $111.7 \pm 1.4$ & & \\
\hline & & & & & & & U-series (MA-10) & $118.8 \pm 1.6$ & & \\
\hline & & & & & & & U-series (MA-11) & $117.2 \pm 1.5$ & & \\
\hline \multirow{6}{*}{780} & \multirow{6}{*}{$\begin{array}{l}39.497035 \\
2.745646\end{array}$} & \multirow{6}{*}{$\begin{array}{l}\text { Cova Baixa (Son } \\
\text { Grauet) }\end{array}$} & \multirow{6}{*}{ SLI } & \multirow{6}{*}{ BRK } & \multirow{6}{*}{$1 \pm 0.3$} & \multirow{6}{*}{$1.6 \pm 1.2$} & U-series (H-C-3) & $129 \pm 7$ & \multirow{6}{*}{4,3} & \multirow{6}{*}{$\begin{array}{l}\text { Lorscheid et al., } 2017 \\
\text { Butzer and Cuerda, } 1962 \\
\text { Hearty et al., 1986a } \\
\text { Hearty, 1987b } \\
\text { Hoang and Hearty, } 1989\end{array}$} \\
\hline & & & & & & & U-series (4878A) & MIS 5e & & \\
\hline & & & & & & & U-series (4878B) & MIS 5e & & \\
\hline & & & & & & & U-series (4878C) & MIS 5e & & \\
\hline & & & & & & & AAR (Son Grauet) & MIS 5e & & \\
\hline & & & & & & & AAR (Son Grauet 2) & MIS 5e & & \\
\hline 781 & $\begin{array}{l}39.488368 \\
2.736524\end{array}$ & Cala Blava & SLI & BRK & $2.7 \pm 0.1$ & $3.3 \pm 1.1$ & U-series (CB90-2) & MIS 5e & 4,3 & $\begin{array}{l}\text { Lorscheid et al., } 2017 \\
\text { Butzer and Cuerda, } 1962 \\
\text { Zazo et al., } 2003\end{array}$ \\
\hline 782 & $\begin{array}{l}39.364069 \\
2.835928\end{array}$ & Cala Pi & SLI & LIT & $3.2 \pm 0.4$ & $4 \pm 0.8$ & AAR (Cala Pi) & MIS 5e & 5,3 & $\begin{array}{l}\text { Lorscheid et al., } 2017 \\
\text { Butzer and Cuerda, } 1962 \\
\text { Hearty, 1987b }\end{array}$ \\
\hline 784 & $\begin{array}{l}39.55927 \\
3.374083\end{array}$ & S'Illot & TLI & & $5.5 \pm 1.7$ & $0 \pm 0$ & AAR (S'Illot) & MIS 5e & 0,3 & $\begin{array}{l}\text { Lorscheid et al., } 2017 \\
\text { Butzer and Cuerda, } 1962 \\
\text { Cuerda, } 1979 \\
\text { Hearty, 1987b }\end{array}$ \\
\hline 785 & $\begin{array}{l}39.59049 \\
3.385249\end{array}$ & Cala Millor & SLI & BRK & $1.5 \pm 0$ & $3.6 \pm 2.8$ & $\begin{array}{l}\text { STRAT ( } P . \text { latus or } \\
\text { Senegalese fauna) }\end{array}$ & MIS 5 & 3,3 & $\begin{array}{l}\text { Lorscheid et al., } 2017 \\
\text { Butzer and Cuerda, } 1962 \\
\text { Cuerda, } 1979 \\
\text { Hearty, 1987b }\end{array}$ \\
\hline
\end{tabular}




\begin{tabular}{|c|c|c|c|c|c|c|c|c|c|c|}
\hline $\begin{array}{l}\text { WALIS } \\
\text { ID }\end{array}$ & $\begin{array}{l}\text { Latitude } \\
\text { Longitude }\end{array}$ & Site & $\begin{array}{l}\text { Type of } \\
\text { datapoint }\end{array}$ & $\begin{array}{l}\text { RSL } \\
\text { Indicator }\end{array}$ & $\begin{array}{l}\text { Elevation } \\
(\mathbf{m})\end{array}$ & $\begin{array}{l}\text { Paleo RSL } \\
\text { (m) }\end{array}$ & Dating (Original ID) & $\begin{array}{l}\text { Age (ka or } \\
\text { MIS) }\end{array}$ & $\begin{array}{l}\text { Quality } \\
\text { (RSL, } \\
\text { Age) }\end{array}$ & Reference(s) \\
\hline 786 & $\begin{array}{l}39.653735 \\
3.439101\end{array}$ & Canyamel & SLI & BRK & $1.3 \pm 0.5$ & $3.3 \pm 2.8$ & AAR (Canyamel) & MIS 5e & 3,3 & $\begin{array}{l}\text { Lorscheid et al., } 2017 \\
\text { Butzer and Cuerda, } 1962 \\
\text { Cuerda, } 1979 \\
\text { Hearty, 1987b }\end{array}$ \\
\hline 787 & $\begin{array}{l}39.748067 \\
3.299692\end{array}$ & Caló des Camps & SLI & IMF & $1.3 \pm 0.1$ & $2.1 \pm 0.7$ & $\begin{array}{l}\text { STRAT ( } P . \text { latus or } \\
\text { Senegalese fauna) }\end{array}$ & MIS 5 & 5,3 & $\begin{array}{l}\text { Lorscheid et al., } 2017 \\
\text { Butzer and Cuerda, } 1962 \\
\text { Cuerda, } 1979 \\
\text { Vicens et al., } 2012\end{array}$ \\
\hline 788 & $\begin{array}{l}39.743077 \\
3.20992 \\
\end{array}$ & Torrent de Son Real & SLI & BRK & $0.9 \pm 0.1$ & $2.2 \pm 2.3$ & $\begin{array}{l}\text { STRAT ( } P . \text { latus or } \\
\text { Senegalese fauna) }\end{array}$ & MIS 5 & 3,3 & $\begin{array}{l}\text { Lorscheid et al., } 2017 \\
\text { Vicens et al., } 2012\end{array}$ \\
\hline 789 & $\begin{array}{l}39.865498 \\
3.136148\end{array}$ & Platja de Sant Joan & SLI & IMF & $2.1 \pm 0.1$ & $2.9 \pm 0.7$ & $\begin{array}{l}\text { STRAT ( } P . \text { latus or } \\
\text { Senegalese fauna) }\end{array}$ & MIS 5 & 5,3 & $\begin{array}{l}\text { Lorscheid et al., } 2017 \\
\text { Cuerda, } 1979 \\
\text { Vicens et al., } 2012\end{array}$ \\
\hline \multicolumn{11}{|c|}{ France (Languedoc-Roussilion) } \\
\hline \multirow[b]{2}{*}{385} & \multirow{2}{*}{$\begin{array}{l}42.89 \\
2.99\end{array}$} & \multirow{2}{*}{$\begin{array}{l}\text { Leucate } \\
\text { Le Malpas }\end{array}$} & \multirow[b]{2}{*}{ SLI } & \multirow[b]{2}{*}{ BRK } & \multirow[b]{2}{*}{$4.4 \pm 0.9$} & \multirow[b]{2}{*}{$4.9 \pm 2.6$} & ESR (YR-C1) & $128 \pm 15$ & \multirow[b]{2}{*}{3,3} & \multirow{2}{*}{$\begin{array}{l}\text { Ambert, } 1999 \\
\text { Yokoyama et al.,1987 }\end{array}$} \\
\hline & & & & & & & $\begin{array}{l}\text { STRAT (P. latus or } \\
\text { Senegalese fauna) }\end{array}$ & MIS 5 & & \\
\hline 1316 & $\begin{array}{l}42.93 \\
3.01 \\
\end{array}$ & $\begin{array}{l}\text { Leucate } \\
\text { La Franqui }\end{array}$ & SLI & BRK & $2.5 \pm 0.5$ & $3 \pm 2.5$ & $\begin{array}{l}\text { OTHER (Limiting } \\
\text { radiocarbon date) }\end{array}$ & >MIS 1 & 3,2 & $\begin{array}{l}\text { Ambert, } 1999 \\
\text { Nesteroff, } 1984\end{array}$ \\
\hline 384 & $\begin{array}{l}42.99 \\
3.03 \\
\end{array}$ & $\begin{array}{l}\text { Port la Nouvelle } \\
\text { Ramandils } \\
\end{array}$ & SLI & BRK & $5.9 \pm 1.2$ & $6.4 \pm 2.8$ & ESR (YR-C1) & $128 \pm 15$ & 3,4 & $\begin{array}{l}\text { Yokoyama et al.,1987 } \\
\text { Ambert, } 1999\end{array}$ \\
\hline \multirow[b]{2}{*}{386} & \multirow{2}{*}{$\begin{array}{l}43.17 \\
3.18\end{array}$} & \multirow{2}{*}{$\begin{array}{l}\text { Narbonne } \\
\text { Saint Pierre }\end{array}$} & \multirow[b]{2}{*}{ SLI } & \multirow[b]{2}{*}{ BRK } & \multirow[b]{2}{*}{$1 \pm 0.2$} & \multirow[b]{2}{*}{$1.5 \pm 2.5$} & ESR (YR-C1) & $128 \pm 15$ & \multirow[b]{2}{*}{3,3} & \multirow{2}{*}{$\begin{array}{l}\text { Ambert, } 1999 \\
\text { Yokoyama et al.,1987 }\end{array}$} \\
\hline & & & & & & & $\begin{array}{l}\text { STRAT ( } P . \text { latus or } \\
\text { Senegalese fauna) }\end{array}$ & MIS 5 & & \\
\hline \multicolumn{11}{|c|}{ France (Provence-Alpes-Cote d'Azur) } \\
\hline 387 & $\begin{array}{l}43.52 \\
5.1 \\
\end{array}$ & Etang de Berre & SLI & LAG & $3 \pm 0.6$ & $3 \pm 1.2$ & U-series (11A) & MIS 5e & 4,3 & Provansal et al., 1995 \\
\hline $\begin{array}{l}1988 \text { (U- } \\
\text { Series } \\
\text { ID) }\end{array}$ & $\begin{array}{l}43.54 \\
7.13\end{array}$ & Cap d'Antibes & SLI & & $6 \pm 0.5$ & 0 to -1 & $\begin{array}{l}\text { U-series (6401- } \\
\text { CalFxAg) }\end{array}$ & $125.5 \pm 5.2$ & N/A & Gilli, 2018 \\
\hline \multirow{6}{*}{449} & \multirow{6}{*}{$\begin{array}{l}43.65 \\
7.15\end{array}$} & \multirow{6}{*}{$\begin{array}{l}\text { Nice } \\
\text { Le Bosquet }\end{array}$} & \multirow{6}{*}{ SLI } & \multirow{6}{*}{ BSD } & \multirow{6}{*}{$16 \pm 0.3$} & \multirow{6}{*}{$14 \pm 1$} & U-series (OS-1) & MIS 5e & \multirow{6}{*}{4,3} & \multirow{6}{*}{ Dubar et al., 2008} \\
\hline & & & & & & & U-series (OS-2) & MIS 5e & & \\
\hline & & & & & & & U-series (OS-3) & MIS 5e & & \\
\hline & & & & & & & U-series (OS-4) & MIS 5e & & \\
\hline & & & & & & & U-series (OS-5) & MIS 5e & & \\
\hline & & & & & & & U-series (OS-6) & MIS 5e & & \\
\hline \multirow{3}{*}{451} & \multirow{3}{*}{$\begin{array}{l}43.69 \\
7.24\end{array}$} & \multirow{3}{*}{$\begin{array}{l}\text { Nice } \\
\text { Les amandier }\end{array}$} & & & & & U-series (SP-1) & MIS 5e & & \\
\hline & & & SLI & BSD & $10 \pm 0.3$ & $10.3 \pm 1.3$ & U-series (SP-2) & MIS 5e & 4,3 & Dubar et al., 2008 \\
\hline & & & & & & & U-series (SP-3) & MIS 5e & & \\
\hline
\end{tabular}




\begin{tabular}{|c|c|c|c|c|c|c|c|c|c|c|}
\hline $\begin{array}{l}\text { WALIS } \\
\text { ID }\end{array}$ & $\begin{array}{l}\text { Latitude } \\
\text { Longitude }\end{array}$ & Site & $\begin{array}{l}\text { Type of } \\
\text { datapoint }\end{array}$ & $\begin{array}{l}\text { RSL } \\
\text { Indicator }\end{array}$ & $\begin{array}{l}\text { Elevation } \\
(\mathbf{m})\end{array}$ & $\begin{array}{l}\text { Paleo RSL } \\
\text { (m) }\end{array}$ & Dating (Original ID) & $\begin{array}{l}\text { Age (ka or } \\
\text { MIS) }\end{array}$ & $\begin{array}{l}\text { Quality } \\
\text { (RSL, } \\
\text { Age) }\end{array}$ & Reference(s) \\
\hline & & & & & & & U-series (SP-4) & MIS 5e & & \\
\hline \multicolumn{11}{|c|}{ Italy (Liguria) } \\
\hline 233 & $\begin{array}{l}43.783733 \\
7.535579\end{array}$ & Barma Grande & SLI & NTC & $11.4 \pm 2.5$ & $11.4 \pm 2.6$ & $\begin{array}{l}\text { STRAT ( } P \text {. latus or } \\
\text { Senegalese fauna) }\end{array}$ & MIS 5 & 3,3 & $\begin{array}{l}\text { Federici and Pappalardo, } 2006 \\
\text { De Lumley, } 1969\end{array}$ \\
\hline 237 & $\begin{array}{l}43.783992 \\
7.536568\end{array}$ & Grotta del Principe & SLI & NTC & $12 \pm 2.4$ & $12 \pm 2.4$ & $\begin{array}{l}\text { STRAT ( } P . \text { latus or } \\
\text { Senegalese fauna) }\end{array}$ & MIS 5 & 3,3 & $\begin{array}{l}\text { Federici and Pappalardo, } 2006 \\
\text { Blanc, } 1955 \\
\text { De Lumley, } 1969\end{array}$ \\
\hline 239 & $\begin{array}{l}43.78363 \\
7.536336\end{array}$ & Bausu da Ture & SLI & BRK & $10.3 \pm 2.2$ & $10.4 \pm 2.3$ & $\begin{array}{l}\text { STRAT (P. latus or } \\
\text { Senegalese fauna) }\end{array}$ & MIS 5 & 3,3 & $\begin{array}{l}\text { Federici and Pappalardo, } 2006 \\
\text { De Lumley, } 1969\end{array}$ \\
\hline 240 & $\begin{array}{l}43.783499 \\
7.53541 \\
\end{array}$ & Ex Casino & SLI & BRK & $7 \pm 1.7$ & $7.2 \pm 1.8$ & $\begin{array}{l}\text { STRAT ( } P . \text { latus or } \\
\text { Senegalese fauna) }\end{array}$ & MIS 5 & 4,3 & $\begin{array}{l}\text { Federici and Pappalardo, } 2006 \\
\text { Vicino, } 1974\end{array}$ \\
\hline 242 & $\begin{array}{l}43.828347 \\
7.845687\end{array}$ & Madonna dell'Arma & SLI & BRK & $7.3 \pm 1.5$ & $7.5 \pm 1.6$ & $\begin{array}{l}\text { STRAT ( } P . \text { latus or } \\
\text { Senegalese fauna) }\end{array}$ & MIS 5 & 4,3 & $\begin{array}{l}\text { Federici and Pappalardo, } 2006 \\
\text { Isetti et al., } 1962\end{array}$ \\
\hline 246 & $\begin{array}{l}44.242635 \\
8.445059\end{array}$ & $\begin{array}{l}\text { Grotta Marina di } \\
\text { Bergeggi }\end{array}$ & SLI & LIT & $5 \pm 0.3$ & $5.2 \pm 0.3$ & $\begin{array}{l}\text { STRAT ( } P . \text { latus or } \\
\text { Senegalese fauna) }\end{array}$ & MIS 5 & 5,3 & $\begin{array}{l}\text { Carobene, } 2015 \\
\text { Oxilia, } 1976 \\
\text { Vicino, } 1978\end{array}$ \\
\hline \multicolumn{11}{|c|}{ Italy (Toscana) } \\
\hline 250 & $\begin{array}{l}43.583 \\
10.367\end{array}$ & Casale Vallino & TLI & & $14 \pm 1.5$ & & $\begin{array}{l}\text { STRAT (P. latus or } \\
\text { Senegalese fauna) }\end{array}$ & MIS 5 & 0,2 & $\begin{array}{l}\text { Nisi et al., } 2003 \\
\text { Ferranti et al., } 2006\end{array}$ \\
\hline 251 & $\begin{array}{l}43.567 \\
10.367\end{array}$ & Pian di Rota & TLI & & $15 \pm 1.5$ & & $\begin{array}{l}\text { STRAT (P. latus or } \\
\text { Senegalese fauna) }\end{array}$ & MIS 5 & 0,2 & $\begin{array}{l}\text { Nisi et al., } 2003 \\
\text { Ferranti et al., } 2006\end{array}$ \\
\hline 252 & $\begin{array}{l}43.55 \\
10.367\end{array}$ & Bagnetti & TLI & & $20 \pm 1.5$ & & $\begin{array}{l}\text { STRAT ( } P . \text { latus or } \\
\text { Senegalese fauna) }\end{array}$ & MIS 5 & 0,2 & $\begin{array}{l}\text { Nisi et al., } 2003 \\
\text { Ferranti et al., } 2006\end{array}$ \\
\hline 253 & $\begin{array}{l}43.5 \\
10.333\end{array}$ & Antignano & TLI & & $20 \pm 1.5$ & & $\begin{array}{l}\text { STRAT (P. latus or } \\
\text { Senegalese fauna) }\end{array}$ & MIS 5 & 0,2 & $\begin{array}{l}\text { Nisi et al., } 2003 \\
\text { Ferranti et al., } 2006\end{array}$ \\
\hline 254 & $\begin{array}{l}43.483 \\
10.333 \\
\end{array}$ & Punta Casotto & TLI & & $15 \pm 1.5$ & & $\begin{array}{l}\text { STRAT ( } P \text {. latus or } \\
\text { Senegalese fauna) }\end{array}$ & MIS 5 & 0,2 & $\begin{array}{l}\text { Nisi et al., } 2003 \\
\text { Ferranti et al., } 2006\end{array}$ \\
\hline 255 & $\begin{array}{l}43.455494 \\
10.372886\end{array}$ & Quercianella & SLI & BRK & $10 \pm 3$ & $10.1 \pm 3.1$ & $\begin{array}{l}\text { STRAT ( } P \text {. latus or } \\
\text { Senegalese fauna) }\end{array}$ & MIS 5 & 2,1 & $\begin{array}{l}\text { Boschian et al., } 2006 \\
\text { Ferranti et al., } 2006 \\
\text { Nisi et al., } 2003\end{array}$ \\
\hline \multirow{2}{*}{325} & \multirow{2}{*}{$\begin{array}{l}43.411509 \\
10.40289\end{array}$} & \multirow{2}{*}{$\begin{array}{l}\text { Buca dei Corvi } \\
\text { BdC1 }\end{array}$} & \multirow{2}{*}{ SLI } & \multirow{2}{*}{ FOR } & \multirow{2}{*}{$9 \pm 2.1$} & \multirow{2}{*}{$9 \pm 2.1$} & AAR (7.BucaDeiCorvi) & MIS 5e & \multirow{2}{*}{3,2} & \multirow{2}{*}{$\begin{array}{l}\text { Mauz, } 1999 \\
\text { Hearty et al., 1986a }\end{array}$} \\
\hline & & & & & & & LUM (BdC 1) & MIS 5d & & \\
\hline 326 & $\begin{array}{l}43.411509 \\
10.40289\end{array}$ & $\begin{array}{l}\text { Buca dei Corvi } \\
\text { BdC2 }\end{array}$ & TLI & & $12 \pm 1.6$ & & LUM (BdC 2) & $94 \pm 34$ & 0,3 & Mauz, 1999 \\
\hline 329 & $\begin{array}{l}43.383 \\
10.45\end{array}$ & Rosignano Solvay & TLI & & $15 \pm 1.5$ & & $\begin{array}{l}\text { STRAT ( } P . \text { latus or } \\
\text { Senegalese fauna) }\end{array}$ & MIS 5 & 0,2 & $\begin{array}{l}\text { Nisi et al., } 2003 \\
\text { Ferranti et al., } 2006\end{array}$ \\
\hline 327 & $\begin{array}{l}42.995168 \\
10.514669 \\
\end{array}$ & $\begin{array}{l}\text { Baratti } \\
\text { Bar 1 }\end{array}$ & SLI & FOR & $1.8 \pm 0.4$ & $1.8 \pm 0.4$ & LUM (Bar 1) & MIS 5d & 5,2 & Mauz, 1999 \\
\hline \multirow{2}{*}{328} & \multirow{2}{*}{$\begin{array}{l}42.995168 \\
10.514669\end{array}$} & \multirow{2}{*}{$\begin{array}{l}\text { Baratti } \\
\text { Bar } 2\end{array}$} & \multirow{2}{*}{ SLI } & \multirow{2}{*}{ BSD } & \multirow{2}{*}{$2.7 \pm 0.8$} & \multirow{2}{*}{$2.6 \pm 0.8$} & LUM (Bar 2a) & $77 \pm 10$ & \multirow{2}{*}{5,4} & \multirow{2}{*}{ Mauz, 1999} \\
\hline & & & & & & & LUM (Bar 2b) & $68 \pm 8$ & & \\
\hline 330 & & & SLI & BRK & $1.7 \pm 0.1$ & $1.8 \pm 0.5$ & AAR (IsolaPianosa) & MIS 5e & 5,4 & \\
\hline
\end{tabular}




\begin{tabular}{|c|c|c|c|c|c|c|c|c|c|c|}
\hline $\begin{array}{l}\text { WALIS } \\
\text { ID }\end{array}$ & $\begin{array}{l}\text { Latitude } \\
\text { Longitude }\end{array}$ & Site & $\begin{array}{l}\text { Type of } \\
\text { datapoint }\end{array}$ & $\begin{array}{l}\text { RSL } \\
\text { Indicator }\end{array}$ & $\begin{array}{l}\text { Elevation } \\
(\mathbf{m})\end{array}$ & $\begin{array}{l}\text { Paleo RSL } \\
\text { (m) }\end{array}$ & Dating (Original ID) & $\begin{array}{l}\text { Age (ka or } \\
\text { MIS) }\end{array}$ & $\begin{array}{l}\text { Quality } \\
\text { (RSL, } \\
\text { Age) }\end{array}$ & Reference(s) \\
\hline & $\begin{array}{l}42.586973 \\
10.100458\end{array}$ & $\begin{array}{l}\text { Pianosa - Cala dei turchi } \\
2\end{array}$ & & & & & $\begin{array}{l}\text { STRAT ( } P . \text { latus or } \\
\text { Senegalese fauna) }\end{array}$ & MIS 5 & & $\begin{array}{l}\text { Stocchi et al, } 2018 \\
\text { Antonioli et al., } 2011 \\
\text { Ferranti et al., } 2006 \\
\text { Hearty and Dai Pra, } 1987\end{array}$ \\
\hline 331 & $\begin{array}{l}42.587368 \\
10.100529\end{array}$ & $\begin{array}{l}\text { Pianosa - Cala dei turchi } \\
1\end{array}$ & SLI & $\mathrm{BIO}$ & $7.9 \pm 1.6$ & $9.3 \pm 2.1$ & $\begin{array}{l}\text { STRAT ( } P \text {. latus or } \\
\text { Senegalese fauna) }\end{array}$ & MIS 5 & 3,2 & $\begin{array}{l}\text { Stocchi et al, } 2018 \\
\text { Antonioli et al., } 2011 \\
\text { Ferranti et al., } 2006 \\
\text { Hearty and Dai Pra, } 1987\end{array}$ \\
\hline 335 & $\begin{array}{l}42.550897 \\
11.165285\end{array}$ & Talamone & SLI & NTC & $4.8 \pm 0.3$ & $4.8 \pm 0.3$ & $\begin{array}{l}\text { STRAT (Mediterranean } \\
\text { MIS 5e notches) }\end{array}$ & MIS 5 & 5,2 & $\begin{array}{l}\text { Antonioli et al., } 2018 \\
\text { Ferranti et al., } 2006 \\
\text { Nisi et al., 2003 }\end{array}$ \\
\hline 336 & $\begin{array}{l}42.533 \\
11.183\end{array}$ & Campo Regio & SLI & BRK & $6 \pm 3.5$ & $6.1 \pm 3.5$ & $\begin{array}{l}\text { STRAT ( } P \text {. latus or } \\
\text { Senegalese fauna) }\end{array}$ & MIS 5 & 2,3 & $\begin{array}{l}\text { Nisi et al., } 2003 \\
\text { Ferranti et al., } 2006 \\
\text { Hearty and Dai Pra, } 1987\end{array}$ \\
\hline \multirow[b]{2}{*}{332} & \multirow{2}{*}{$\begin{array}{l}42.411 \\
11.406\end{array}$} & \multirow[b]{2}{*}{ Selva Nera } & \multirow[b]{2}{*}{ SLI } & \multirow[b]{2}{*}{ BRK } & \multirow[b]{2}{*}{$10 \pm 1.5$} & \multirow[b]{2}{*}{$10.2 \pm 1.6$} & AAR (9.Selvanera) & MIS 5e & \multirow[b]{2}{*}{3,3} & \multirow{2}{*}{$\begin{array}{l}\text { Hearty and Dai Pra, } 1987 \\
\text { Bordoni and Valensise, } 1999 \\
\text { Ferranti et al., } 2006 \\
\text { Nisi et al., 2003 }\end{array}$} \\
\hline & & & & & & & AAR (9.Selvanera_2) & MIS 5e & & \\
\hline 334 & $\begin{array}{l}42.407086 \\
11.448872\end{array}$ & San Angelino & SLI & BRK & $12 \pm 1.5$ & $12.2 \pm 1.6$ & AAR (11.SanAngelino) & MIS 5e & 4,3 & $\begin{array}{l}\text { Hearty and Dai Pra, } 1987 \\
\text { Ferranti et al., } 2006 \\
\text { Nisi et al., 2003 }\end{array}$ \\
\hline 333 & $\begin{array}{l}42.398721 \\
11.447669 \\
\end{array}$ & Vado Piano & SLI & BRK & $13 \pm 2.6$ & $13.2 \pm 2.7$ & AAR (10.Vadopiano) & MIS 5e & 2,3 & $\begin{array}{l}\text { Hearty and Dai Pra, } 1987 \\
\text { Ferranti et al., } 2006\end{array}$ \\
\hline \multicolumn{11}{|c|}{ Italy (Lazio) } \\
\hline \multirow{3}{*}{354} & \multirow{3}{*}{$\begin{array}{l}42.3831 \\
11.4968\end{array}$} & \multirow{3}{*}{ Lasco Del Pozzo } & \multirow{3}{*}{ SLI } & \multirow{3}{*}{ BRK } & \multirow{3}{*}{$7 \pm 1.5$} & \multirow{3}{*}{$7.2 \pm 1.6$} & $\begin{array}{l}\text { AAR } \\
\text { (12.LascoDelPozzo_1) }\end{array}$ & MIS 5e & \multirow{3}{*}{4,3} & \multirow{3}{*}{$\begin{array}{l}\text { Hearty and Dai Pra, } 1987 \\
\text { Bordoni and Valensise, } 1999 \\
\text { Ferranti et al., } 2006 \\
\text { Nisi et al., } 2003\end{array}$} \\
\hline & & & & & & & $\begin{array}{l}\text { AAR } \\
\text { (12.LascoDelPozzo_2) }\end{array}$ & MIS 5e & & \\
\hline & & & & & & & $\begin{array}{l}\text { AAR } \\
\text { (12.LascoDelPozzo_3) }\end{array}$ & MIS 5e & & \\
\hline 475 & $\begin{array}{l}42.3618 \\
11.5394\end{array}$ & $\begin{array}{l}\text { Centrale Nucleare } \\
\text { Montalto di Castro }\end{array}$ & SLI & BRK & $4.4 \pm 0.9$ & $4.6 \pm 1.1$ & $\begin{array}{l}\text { AAR (13.Centrale } \\
\text { Nucleare Montalto) }\end{array}$ & MIS 5e & 4,3 & Hearty and Dai Pra, 1987 \\
\hline 476 & $\begin{array}{l}42.3677 \\
11.5475 \\
\end{array}$ & La Ficonaccia & SLI & BRK & $12 \pm 2.4$ & $12.2 \pm 2.5$ & $\begin{array}{l}\text { AAR (14.La } \\
\text { Ficonaccia) }\end{array}$ & MIS 5e & 1,3 & Hearty and Dai Pra, 1987 \\
\hline \multirow{2}{*}{477} & \multirow{2}{*}{$\begin{array}{l}42.3723 \\
11.5554\end{array}$} & \multirow{2}{*}{ Ponte Rotto } & \multirow{2}{*}{ SLI } & \multirow{2}{*}{ BRK } & \multirow{2}{*}{$14 \pm 2.8$} & \multirow{2}{*}{$14.2 \pm 2.9$} & $\begin{array}{l}\text { AAR (15.Ponte } \\
\text { Rotto.2) }\end{array}$ & MIS 5e & \multirow{2}{*}{1,3} & \multirow{2}{*}{ Hearty and Dai Pra, 1987} \\
\hline & & & & & & & $\begin{array}{l}\text { AAR (15.Ponte } \\
\text { Rotto.1) }\end{array}$ & MIS 5e & & \\
\hline 478 & $\begin{array}{l}42.382 \\
11.5556\end{array}$ & Km 115.5 & SLI & BRK & $20 \pm 1.5$ & $20.2 \pm 1.6$ & AAR (15.Km115.5) & MIS 5e & 1,3 & $\begin{array}{l}\text { Hearty and Dai Pra, } 1987 \\
\text { Bordoni and Valensise, } 1999 \\
\text { Ferranti et al., 2006 } \\
\text { Nisi et al., 2003 } \\
\end{array}$ \\
\hline \multirow{2}{*}{482} & \multirow{2}{*}{$\begin{array}{l}42.316667 \\
11.642\end{array}$} & \multirow{2}{*}{$\begin{array}{l}\text { Il Mandrione, right bank } \\
\text { of Arrone River }\end{array}$} & \multirow{2}{*}{ SLI } & \multirow{2}{*}{ BRK } & \multirow{2}{*}{$24 \pm 4.8$} & $242+48$ & $\begin{array}{l}\text { STRAT ( } P . \text { latus or } \\
\text { Senegalese fauna) }\end{array}$ & MIS 5 & 21 & $\begin{array}{l}\text { Palieri and Sposato, } 1988 \\
\text { Bordoni and Valensise, } 1999\end{array}$ \\
\hline & & & & & & $24.2 \pm 4.0$ & OTHER (MAND 1 B) & $<255$ & 2,1 & $\begin{array}{l}\text { Marra et al., } 2019 \\
\text { Nisi et al., } 2003\end{array}$ \\
\hline
\end{tabular}




\begin{tabular}{|c|c|c|c|c|c|c|c|c|c|c|}
\hline $\begin{array}{l}\text { WALIS } \\
\text { ID }\end{array}$ & $\begin{array}{l}\text { Latitude } \\
\text { Longitude }\end{array}$ & Site & $\begin{array}{l}\text { Type of } \\
\text { datapoint }\end{array}$ & $\begin{array}{l}\text { RSL } \\
\text { Indicator }\end{array}$ & $\begin{array}{l}\text { Elevation } \\
\text { (m) }\end{array}$ & $\begin{array}{l}\text { Paleo RSL } \\
\text { (m) }\end{array}$ & Dating (Original ID) & $\begin{array}{l}\text { Age (ka or } \\
\text { MIS) }\end{array}$ & $\begin{array}{l}\text { Quality } \\
\text { (RSL, } \\
\text { Age) }\end{array}$ & Reference(s) \\
\hline \multirow{4}{*}{4070} & \multirow{4}{*}{$\begin{array}{l}42.3077 \\
11.6478\end{array}$} & \multirow{4}{*}{ Riva Dei Tarquini } & \multirow{4}{*}{ SLI } & \multirow{4}{*}{ MT } & \multirow{4}{*}{$25 \pm 5$} & \multirow{4}{*}{$24.9 \pm 5.1$} & U-series (RTAR(1)-IN) & MIS 5 & \multirow{4}{*}{3,4} & \multirow{4}{*}{$\begin{array}{l}\text { Radtke, } 1983 \\
\text { Radtke, } 1986\end{array}$} \\
\hline & & & & & & & $\begin{array}{l}\text { U-series (RTAR(1)- } \\
\text { OUT) }\end{array}$ & MIS 5 & & \\
\hline & & & & & & & ESR (RTAR(1)-IN) & $102 \pm 20.4$ & & \\
\hline & & & & & & & ESR (RTAR(1)-OUT) & $112 \pm 22.4$ & & \\
\hline 481 & $\begin{array}{l}42.29 \\
11.67\end{array}$ & $\begin{array}{l}\text { Between Arrone and } \\
\text { Mignone rivers }\end{array}$ & SLI & BRK & $20 \pm 4$ & $20.2 \pm 4$ & $\begin{array}{l}\text { STRAT ( } P . \text { latus or } \\
\text { Senegalese fauna) }\end{array}$ & MIS 5 & 2,3 & $\begin{array}{l}\text { Bordoni and Valensise, } 1999 \\
\text { Bonadonna, } 1967 \\
\text { Gignoux, } 1913\end{array}$ \\
\hline \multirow{2}{*}{483} & \multirow{2}{*}{$\begin{array}{l}42.289 \\
11.682\end{array}$} & \multirow{2}{*}{ Aurelia km 103} & \multirow{2}{*}{ SLI } & \multirow{2}{*}{ BRK } & \multirow{2}{*}{$25 \pm 5$} & \multirow{2}{*}{$25.2 \pm 5$} & AAR (5.KM103.1) & MIS 5e & \multirow{2}{*}{2,3} & \multirow{2}{*}{$\begin{array}{l}\text { Hearty and Dai Pra, } 1986 \\
\text { Bordoni and Valensise, } 1999 \\
\text { Nisi et al., } 2003\end{array}$} \\
\hline & & & & & & & AAR (5.KM103.2) & MIS 5e & & \\
\hline 484 & $\begin{array}{l}42.23 \\
11.73\end{array}$ & $\begin{array}{l}\text { Northwest of Tarquinia } \\
\text { railway station }\end{array}$ & SLI & BRK & $13 \pm 2.8$ & $13.2 \pm 2.9$ & $\begin{array}{l}\text { STRAT ( } P . \text { latus or } \\
\text { Senegalese fauna) }\end{array}$ & MIS 5 & 3,3 & $\begin{array}{l}\text { Palieri and Sposato, } 1988 \\
\text { Bordoni and Valensise, } 1999 \\
\text { Nisi et al., } 2003\end{array}$ \\
\hline \multirow{2}{*}{4071} & \multirow{2}{*}{$\begin{array}{l}42.18628 \\
11.74356\end{array}$} & \multirow{2}{*}{$\mathrm{Km} 17-1(\mathrm{k})$} & \multirow{2}{*}{ MLI } & & \multirow{2}{*}{$8.7 \pm 2.1$} & & U-series $(\mathrm{Km} 17-1(\mathrm{k})$ & MIS 5 & \multirow{2}{*}{0,3} & \multirow{2}{*}{$\begin{array}{l}\text { Radtke, } 1983 \\
\text { Radtke, } 1986\end{array}$} \\
\hline & & & & & & & ESR $(\mathrm{Km} 17-1(\mathrm{k})$ & $79 \pm 15.8$ & & \\
\hline \multirow{2}{*}{479} & \multirow{2}{*}{$\begin{array}{l}42.216 \\
11.7461\end{array}$} & \multirow{2}{*}{ F. delle Serpe } & \multirow{2}{*}{ SLI } & \multirow{2}{*}{ BRK } & \multirow{2}{*}{$12 \pm 2.4$} & \multirow{2}{*}{$12.2 \pm 2.5$} & $\begin{array}{l}\text { AAR (24.F. delle } \\
\text { Serpe) }\end{array}$ & MIS 5e & 12 & Hentw and Doi Dra 1087 \\
\hline & & & & & & & $\begin{array}{l}\text { STRAT ( } P . \text { latus or } \\
\text { Senegalese fauna) }\end{array}$ & MIS 5 & 1,3 & Hearty and Da1 Pra, 1987 \\
\hline 480 & 42.1859 & & & & & & $\begin{array}{l}\text { AAR (26. C.le } \\
\text { Olivastro) }\end{array}$ & MIS 5e & & $\begin{array}{l}\text { Hearty and Dai Pra, } 1987 \\
\text { Bonadonna, } 1967\end{array}$ \\
\hline 480 & 11.7453 & Casale Olivastro & SLI & BRK & $16 \pm 3$ & $16.2 \pm 3.1$ & $\begin{array}{l}\text { STRAT (P. latus or } \\
\text { Senegalese fauna) }\end{array}$ & MIS 5 & 1,3 & $\begin{array}{l}\text { Ferranti et al., } 2006 \\
\text { Nisi et al., } 2003\end{array}$ \\
\hline & & & & & & & U-series (CSV-P-1) & MIS 5 & & \\
\hline & 42.20493 & Casal San Vincenzo & SLI & BRK & $33+21$ & $332+21$ & U-series (CSV-P-2) & MIS 5 & 24 & Radtke, 1983 \\
\hline 4072 & 11.79407 & $(\mathrm{CSV})$ & SLI & BRK & $33 \pm 2.1$ & $33.2 \pm 2.1$ & ESR (CSV-P-1) & $111 \pm 22.2$ & 2,4 & Radtke, 1986 \\
\hline & & & & & & & ESR (CSV-P-2) & $92 \pm 18.4$ & & \\
\hline 494 & $\begin{array}{l}42.11 \\
11.811\end{array}$ & Monna Felice & SLI & MT & $35 \pm 1.5$ & $34.9 \pm 1.7$ & $\begin{array}{l}\text { STRAT ( } P \text {. latus or } \\
\text { Senegalese fauna) }\end{array}$ & MIS 5 & 4,3 & $\begin{array}{l}\text { Bordoni and Valensise, } 1999 \\
\text { Ferranti et al., } 2006 \\
\text { Nisi et al., 2003 } \\
\text { Benjamin et al., } 2017 \\
\text { Gignoux, 1913 } \\
\text { Taviani, 2014 } \\
\text { Zazo et al., } 2013 \\
\end{array}$ \\
\hline & & & & & & & $\begin{array}{l}\text { AAR (4.Cerveteri- } \\
\text { Monteroni 1) }\end{array}$ & & & Hearty and Dai Pra, 1986 \\
\hline 495 & $\begin{array}{l}41.994 \\
12.109\end{array}$ & Monteroni - Cerveteri & SLI & LAG & $27 \pm 1.5$ & $28.1 \pm 1.8$ & $\begin{array}{l}\text { AAR (4.Cerveteri- } \\
\text { Monteroni 2) }\end{array}$ & MIS 5e & 4,3 & $\begin{array}{l}\text { Bordoni and Valensise, } 1999 \\
\text { Ferranti et al., } 2006\end{array}$ \\
\hline & & & & & & & $\begin{array}{l}\text { AAR (4.Cerveteri- } \\
\text { Monteroni 3) }\end{array}$ & & & Nisi et al., 2003 \\
\hline
\end{tabular}




\begin{tabular}{|c|c|c|c|c|c|c|c|c|c|c|}
\hline $\begin{array}{l}\text { WALIS } \\
\text { ID }\end{array}$ & $\begin{array}{l}\text { Latitude } \\
\text { Longitude }\end{array}$ & Site & $\begin{array}{l}\text { Type of } \\
\text { datapoint }\end{array}$ & $\begin{array}{l}\text { RSL } \\
\text { Indicator }\end{array}$ & $\begin{array}{l}\text { Elevation } \\
(\mathbf{m})\end{array}$ & $\begin{array}{l}\text { Paleo RSL } \\
\text { (m) }\end{array}$ & Dating (Original ID) & $\begin{array}{l}\text { Age (ka or } \\
\text { MIS) }\end{array}$ & $\begin{array}{l}\text { Quality } \\
\text { (RSL, } \\
\text { Age) }\end{array}$ & Reference(s) \\
\hline 496 & $\begin{array}{l}41.933 \\
12.207\end{array}$ & Casale di Statua & SLI & BRK & $19 \pm 3.8$ & $19.2 \pm 3.9$ & $\begin{array}{l}\text { AAR (3. Palidoro } \\
\text { (Blanc) }\end{array}$ & MIS 5e & 2,3 & $\begin{array}{l}\text { Hearty and Dai Pra, } 1986 \\
\text { Bordoni and Valensise, } 1999 \\
\text { Ferranti et al., } 2006 \\
\text { Nisi et al., 2003 } \\
\end{array}$ \\
\hline 529 & $\begin{array}{l}41.824314 \\
12.331983 \\
\end{array}$ & Cava Rinaldi (point d) & TLI & & $26.1 \pm 0.02$ & & OTHER (NCR2) & MIS 5e & 0,4 & $\begin{array}{l}\text { Marra et al., } 2016 \\
\text { Marra et al., } 2019\end{array}$ \\
\hline \multirow[b]{2}{*}{527} & \multirow{2}{*}{$\begin{array}{l}41.4571 \\
12.7742\end{array}$} & \multirow[b]{2}{*}{ Quadrato } & \multirow[b]{2}{*}{ SLI } & \multirow[b]{2}{*}{ BRK } & \multirow[b]{2}{*}{$11 \pm 2.4$} & \multirow[b]{2}{*}{$11.2 \pm 2.5$} & ESR (QDT 1601) & $79 \pm 7$ & \multirow[b]{2}{*}{1,1} & \multirow[b]{2}{*}{ Marra et al., 2019} \\
\hline & & & & & & & $\begin{array}{l}\text { STRAT ( } P \text {. latus or } \\
\text { Senegalese fauna) }\end{array}$ & MIS 5 & & \\
\hline 497 & $\begin{array}{l}41.46 \\
12.81\end{array}$ & $\begin{array}{l}\text { Borgo Santa Maria, west } \\
\text { of Latina }\end{array}$ & SLI & BRK & $12 \pm 2.4$ & $12.2 \pm 2.5$ & $\begin{array}{l}\text { AAR (15. Casale } \\
\text { Nuovo - Borgo S. } \\
\text { Maria) }\end{array}$ & MIS 5e & 3,3 & $\begin{array}{l}\text { Hearty and Dai Pra, } 1986 \\
\text { Bordoni and Valensise, } 1999\end{array}$ \\
\hline \multirow{3}{*}{498} & \multirow{3}{*}{$\begin{array}{l}41.43 \\
12.82\end{array}$} & \multirow{3}{*}{ Borgo Sabotino } & \multirow{3}{*}{ SLI } & \multirow{3}{*}{ BRK } & \multirow{3}{*}{$10 \pm 3.5$} & \multirow{3}{*}{$10.2 \pm 3.6$} & $\begin{array}{l}\text { AAR (16.Borgo } \\
\text { Sabotino A) }\end{array}$ & \multirow{3}{*}{ MIS 5e } & \multirow{3}{*}{2,3} & \multirow{3}{*}{$\begin{array}{l}\text { Hearty and Dai Pra, } 1986 \\
\text { Bordoni and Valensise, } 1999 \\
\text { Ferranti et al., } 2006 \\
\text { Nisi et al., } 2003\end{array}$} \\
\hline & & & & & & & $\begin{array}{l}\text { AAR (16.Borgo } \\
\text { Sabotino B-C) }\end{array}$ & & & \\
\hline & & & & & & & $\begin{array}{l}\text { AAR (16.Borgo } \\
\text { Sabotino B-C 2) }\end{array}$ & & & \\
\hline 513 & $\begin{array}{l}41.417 \\
13.05\end{array}$ & Pontinia I & SLI & LAG & $5.3 \pm 2$ & $6.4 \pm 2.2$ & $\begin{array}{l}\text { STRAT (Mediterranean } \\
\text { AAR Aminozone E) }\end{array}$ & MIS 5e & 3,3 & $\begin{array}{l}\text { Antonioli et al., 1999b } \\
\text { Ferranti et al., } 2006 \\
\text { Nisi et al., 2003 }\end{array}$ \\
\hline 514 & $\begin{array}{l}41.417 \\
13.067\end{array}$ & Pontinia II & SLI & LAG & $4.4 \pm 2$ & $5.5 \pm 2.2$ & $\begin{array}{l}\text { STRAT (Mediterranean } \\
\text { AAR Aminozone E) }\end{array}$ & MIS 5e & 3,3 & $\begin{array}{l}\text { Antonioli et al., 1999b } \\
\text { Ferranti et al., } 2006 \\
\text { Nisi et al., } 2003\end{array}$ \\
\hline 515 & $\begin{array}{l}41.433 \\
13.067\end{array}$ & Pontinia III & SLI & LAG & $2.3 \pm 2$ & $3.4 \pm 2.2$ & $\begin{array}{l}\text { STRAT (Mediterranean } \\
\text { AAR Aminozone E) }\end{array}$ & MIS 5e & 3,3 & $\begin{array}{l}\text { Antonioli et al., 1999b } \\
\text { Ferranti et al., } 2006 \\
\text { Nisi et al., } 2003\end{array}$ \\
\hline 516 & $\begin{array}{l}41.433 \\
13.083\end{array}$ & Pontinia IV & SLI & LAG & $0.8 \pm 2$ & $1.9 \pm 2.2$ & $\begin{array}{l}\text { STRAT (Mediterranean } \\
\text { AAR Aminozone E) }\end{array}$ & MIS 5e & 3,3 & $\begin{array}{l}\text { Antonioli et al., 1999b } \\
\text { Ferranti et al., } 2006 \\
\text { Nisi et al., } 2003\end{array}$ \\
\hline 517 & $\begin{array}{l}41.45 \\
13.083\end{array}$ & Pontinia V & SLI & LAG & $-0.5 \pm 2$ & $0.6 \pm 2.2$ & $\begin{array}{l}\text { STRAT (Mediterranean } \\
\text { AAR Aminozone E) }\end{array}$ & MIS 5e & 3,3 & $\begin{array}{l}\text { Antonioli et al., 1999b } \\
\text { Ferranti et al., } 2006 \\
\text { Nisi et al., } 2003\end{array}$ \\
\hline 518 & $\begin{array}{l}41.45 \\
13.1\end{array}$ & Pontinia VI & SLI & LAG & $-15.5 \pm 5$ & $-14.4 \pm 5.1$ & $\begin{array}{l}\text { STRAT (Mediterranean } \\
\text { AAR Aminozone E) }\end{array}$ & MIS 5e & 2,3 & $\begin{array}{l}\text { Antonioli et al., 1999b } \\
\text { Ferranti et al., } 2006 \\
\text { Nisi et al., } 2003\end{array}$ \\
\hline 519 & $\begin{array}{l}41.35 \\
13.117\end{array}$ & Borgo Vodice I & SLI & LAG & $1 \pm 2$ & $2.1 \pm 2.2$ & $\begin{array}{l}\text { STRAT (Mediterranean } \\
\text { AAR Aminozone E) }\end{array}$ & MIS 5e & 3,3 & $\begin{array}{l}\text { Antonioli et al., 1999b } \\
\text { Ferranti et al., } 2006 \\
\text { Nisi et al., 2003 }\end{array}$ \\
\hline 520 & $\begin{array}{l}41.35 \\
13.117\end{array}$ & Borgo Vodice II & SLI & LAG & $-0.6 \pm 2$ & $0.5 \pm 2.2$ & $\begin{array}{l}\text { STRAT (Mediterranean } \\
\text { AAR Aminozone E) }\end{array}$ & MIS 5e & 3,3 & $\begin{array}{l}\text { Antonioli et al., 1999b } \\
\text { Ferranti et al., } 2006 \\
\text { Nisi et al., } 2003\end{array}$ \\
\hline 521 & $\begin{array}{l}41.35 \\
13.117\end{array}$ & Borgo Vodice III & SLI & LAG & $-1.8 \pm 2$ & $-0.7 \pm 2.2$ & $\begin{array}{l}\text { STRAT (Mediterranean } \\
\text { AAR Aminozone E) }\end{array}$ & MIS 5e & 3,3 & $\begin{array}{l}\text { Antonioli et al., 1999b } \\
\text { Ferranti et al., } 2006 \\
\text { Nisi et al., } 2003\end{array}$ \\
\hline
\end{tabular}




\begin{tabular}{|c|c|c|c|c|c|c|c|c|c|c|}
\hline $\begin{array}{l}\text { WALIS } \\
\text { ID }\end{array}$ & $\begin{array}{l}\text { Latitude } \\
\text { Longitude }\end{array}$ & Site & $\begin{array}{l}\text { Type of } \\
\text { datapoint }\end{array}$ & $\begin{array}{l}\text { RSL } \\
\text { Indicator }\end{array}$ & $\begin{array}{l}\text { Elevation } \\
(\mathbf{m})\end{array}$ & $\begin{array}{l}\text { Paleo RSL } \\
\text { (m) }\end{array}$ & Dating (Original ID) & $\begin{array}{l}\text { Age (ka or } \\
\text { MIS) }\end{array}$ & $\begin{array}{l}\text { Quality } \\
\text { (RSL, } \\
\text { Age) }\end{array}$ & Reference(s) \\
\hline \multirow{3}{*}{499} & \multirow{3}{*}{$\begin{array}{l}41.224 \\
13.065\end{array}$} & \multirow{3}{*}{ Torre Rossa } & \multirow{3}{*}{ MLI } & & \multirow{3}{*}{$3 \pm 0.6$} & & AAR (Torre Rossa 1) & \multirow{3}{*}{ MIS 5e } & \multirow{3}{*}{0,3} & \multirow{3}{*}{$\begin{array}{l}\text { Hearty and Dai Pra, } 1986 \\
\text { Bordoni and Valensise, } 1999\end{array}$} \\
\hline & & & & & & & AAR (Torre Rossa 2) & & & \\
\hline & & & & & & & AAR (Torre Rossa 3) & & & \\
\hline 522 & $\begin{array}{l}41.223901 \\
13.082592\end{array}$ & $\begin{array}{l}\text { Circeo (Grotta delle } \\
\text { Capre) }\end{array}$ & SLI & NTC & $9.3 \pm 0.2$ & $9.3 \pm 0.2$ & $\begin{array}{l}\text { STRAT (Mediterranean } \\
\text { MIS 5e notches) }\end{array}$ & MIS 5 & 5,2 & $\begin{array}{l}\text { Antonioli et al., } 2018 \\
\text { Ferranti et al., } 2006 \\
\text { Nisi et al., } 2003\end{array}$ \\
\hline 523 & $\begin{array}{l}41.288243 \\
13.260035\end{array}$ & $\begin{array}{l}\text { Terracina (Pisco } \\
\text { Montano) }\end{array}$ & SLI & NTC & $8 \pm 0.2$ & $8 \pm 0.2$ & $\begin{array}{l}\text { STRAT (Mediterranean } \\
\text { MIS 5e notches) }\end{array}$ & MIS 5 & 5,2 & $\begin{array}{l}\text { Antonioli et al., } 2018 \\
\text { Ferranti et al., } 2006 \\
\text { Nisi et al., } 2003 \\
\end{array}$ \\
\hline \multirow{3}{*}{508} & \multirow{3}{*}{$\begin{array}{l}41.302 \\
13.338\end{array}$} & \multirow{3}{*}{ Fondi plain core ATP4 } & \multirow{3}{*}{ SLI } & \multirow{3}{*}{ LAG } & \multirow{3}{*}{$-6 \pm 2$} & \multirow{3}{*}{$-4.9 \pm 2.2$} & AAR (ATP4 1) & MIS 5e & \multirow{3}{*}{2,1} & \multirow{3}{*}{$\begin{array}{l}\text { Antonioli et al., } 1988 \\
\text { Bordoni and Valensise, } 1999 \\
\text { Ferranti et al., } 2006 \\
\text { Nisi et al., } 2003\end{array}$} \\
\hline & & & & & & & AAR (ATP4 2) & MIS 5e & & \\
\hline & & & & & & & AAR (ATP4 3) & MIS 5e & & \\
\hline 500 & $\begin{array}{l}41.244 \\
13.471 \\
\end{array}$ & Torre Capovento & SLI & BRK & $5 \pm 1$ & $5.2 \pm 1.2$ & $\begin{array}{l}\text { AAR (Torre } \\
\text { Capovento) }\end{array}$ & MIS 5e & 2,1 & $\begin{array}{l}\text { Hearty and Dai Pra, } 1986 \\
\text { Bordoni and Valensise, } 1999\end{array}$ \\
\hline 524 & $\begin{array}{l}41.21845 \\
13.532598\end{array}$ & $\begin{array}{l}\text { Sperlonga } \\
\text { (Sant'agostino) }\end{array}$ & SLI & NTC & $6.5 \pm 0.2$ & $6.5 \pm 0.2$ & $\begin{array}{l}\text { STRAT (Mediterranean } \\
\text { MIS 5e notches) }\end{array}$ & MIS 5 & 5,2 & $\begin{array}{l}\text { Antonioli et al., } 2018 \\
\text { Ferranti et al., } 2006 \\
\text { Nisi et al., 2003 }\end{array}$ \\
\hline 525 & $\begin{array}{l}41.204594 \\
13.57162 \\
\end{array}$ & Gaeta (Grotta del Turco) & SLI & NTC & $5.9 \pm 0.3$ & $5.9 \pm 0.3$ & $\begin{array}{l}\text { STRAT (Mediterranean } \\
\text { MIS 5e notches) }\end{array}$ & MIS 5 & 5,2 & $\begin{array}{l}\text { Antonioli et al., } 2018 \\
\text { Ferranti et al., } 2006 \\
\text { Nisi et al., 2003 }\end{array}$ \\
\hline 501 & $\begin{array}{l}41.268 \\
13.715 \\
\end{array}$ & Marina di Minturno & SLI & BRK & $8 \pm 1.6$ & $8.2 \pm 1.7$ & $\begin{array}{l}\text { AAR (20. Marina di } \\
\text { Minturno) }\end{array}$ & MIS 5e & 2,1 & $\begin{array}{l}\text { Hearty and Dai Pra, } 1986 \\
\text { Bordoni and Valensise, } 1999\end{array}$ \\
\hline 526 & $\begin{array}{l}41.239725 \\
13.736821\end{array}$ & $\begin{array}{l}\text { Minturno (Monte } \\
\text { d'Argento) }\end{array}$ & SLI & NTC & $12.5 \pm 0.2$ & $12.5 \pm 0.2$ & $\begin{array}{l}\text { STRAT (Mediterranean } \\
\text { MIS 5e notches) }\end{array}$ & MIS 5 & 5,2 & $\begin{array}{l}\text { Antonioli et al., } 2018 \\
\text { Ferranti et al., } 2006 \\
\text { Nisi et al., } 2003 \\
\end{array}$ \\
\hline \multicolumn{11}{|c|}{ Italy (Campania) } \\
\hline 3624 & $\begin{array}{l}41.201997 \\
13.832 \\
\end{array}$ & $\begin{array}{l}\text { Cellole Aurunci } \\
\text { Masseria Transitiello }\end{array}$ & MLI & & $6 \pm 1.2$ & $6 \pm 1.2$ & $\begin{array}{l}\text { AAR (Cellole Aurunci } \\
\text { (Masseria Transitiello) }\end{array}$ & $130 \pm 39$ & 0,3 & Brancaccio et al., 1990 \\
\hline \multirow{2}{*}{722} & \multirow{2}{*}{$\begin{array}{l}40.991003 \\
14.175998\end{array}$} & \multirow{2}{*}{ San Marcellino well } & \multirow{2}{*}{ MLI } & & \multirow{2}{*}{$-50 \pm 1$} & \multirow{2}{*}{$0 \pm 1$} & U-series (1122a) & $138 \pm 10$ & \multirow{2}{*}{0,4} & \multirow{2}{*}{ Romano et al., 1994} \\
\hline & & & & & & & U-series (1122b) & $137 \pm 11$ & & \\
\hline \multirow[b]{2}{*}{739} & \multirow{2}{*}{$\begin{array}{l}41.026523 \\
14.3394\end{array}$} & \multirow[b]{2}{*}{ San Marco Evangelista } & \multirow[b]{2}{*}{ MLI } & & \multirow[b]{2}{*}{$-18 \pm 1$} & & STRAT (Sep 4) & $>$ MIS $5 c$ & \multirow[b]{2}{*}{0,2} & \\
\hline & & & & & & & $\begin{array}{l}\text { OTHER (San Marco } \\
\text { Evangelista) }\end{array}$ & $<$ MIS 7 & & Santangelo et al., 2010 \\
\hline & & & & & & & U-series (N/A) & $217 \pm 65.1$ & & \\
\hline 790 & 14.649142 & Sarno well & MLI & & $-23 \pm 1$ & & $\begin{array}{l}\text { STRAT (Sylvestra } \\
\text { seminis) }\end{array}$ & MIS 5e & 0,3 & Barra et al., 1991 \\
\hline 3581 & 40.586558 & Mitioliano & & NTC & $5+03$ & & U-series (C-1) & $128 \pm 5$ & & Antonioli et al., 2018 \\
\hline 5501 & 14.327083 & Mitigilano & SLI & NIC & $3 \pm 0.3$ & $5 \pm 0.3$ & U-series (C-3) & $131 \pm 6$ & 5,2 & Brancaccio et al., 1978 \\
\hline
\end{tabular}




\begin{tabular}{|c|c|c|c|c|c|c|c|c|c|c|}
\hline $\begin{array}{l}\text { WALIS } \\
\text { ID }\end{array}$ & $\begin{array}{l}\text { Latitude } \\
\text { Longitude }\end{array}$ & Site & $\begin{array}{l}\text { Type of } \\
\text { datapoint }\end{array}$ & $\begin{array}{l}\text { RSL } \\
\text { Indicator }\end{array}$ & $\begin{array}{l}\text { Elevation } \\
(\mathbf{m})\end{array}$ & $\begin{array}{l}\text { Paleo RSL } \\
\text { (m) }\end{array}$ & Dating (Original ID) & $\begin{array}{l}\text { Age (ka or } \\
\text { MIS) }\end{array}$ & $\begin{array}{l}\text { Quality } \\
\text { (RSL, } \\
\text { Age) }\end{array}$ & Reference(s) \\
\hline & & & & & & & U-series (C-4) & $133 \pm 6$ & & \\
\hline & & & & & & & U-series (C-5) & $123 \pm 8$ & & \\
\hline \multirow{4}{*}{821} & \multirow{4}{*}{$\begin{array}{l}40.559447 \\
14.256813\end{array}$} & \multirow{4}{*}{$\begin{array}{l}\text { Scoglio Ricotta (I) } \\
\text { Capri }\end{array}$} & \multirow{4}{*}{ SLI } & \multirow{4}{*}{ NTC } & \multirow{4}{*}{$6.8 \pm 0.1$} & \multirow{4}{*}{$6.8 \pm 0.2$} & U-series (C-1) & $128 \pm 5$ & \multirow{4}{*}{5,2} & \multirow{4}{*}{$\begin{array}{l}\text { Ferranti \& Antonioli, } 2007 \\
\text { Brancaccio et al., } 1978\end{array}$} \\
\hline & & & & & & & U-series (C-3) & $131 \pm 6$ & & \\
\hline & & & & & & & U-series (C-4) & $133 \pm 6$ & & \\
\hline & & & & & & & U-series (C-5) & $123 \pm 8$ & & \\
\hline \multirow{4}{*}{822} & \multirow{4}{*}{$\begin{array}{l}40.558063 \\
14.255372\end{array}$} & \multirow{4}{*}{$\begin{array}{l}\text { Scoglio Ricotta (II) } \\
\text { Capri }\end{array}$} & \multirow{4}{*}{ SLI } & \multirow{4}{*}{ NTC } & \multirow{4}{*}{$6 \pm 0.1$} & \multirow{4}{*}{$6 \pm 0.2$} & U-series (C-1) & $128 \pm 5$ & \multirow{4}{*}{5,2} & \multirow{4}{*}{$\begin{array}{l}\text { Ferranti \& Antonioli, } 2007 \\
\text { Brancaccio et al., } 1978\end{array}$} \\
\hline & & & & & & & U-series (C-3) & $131 \pm 6$ & & \\
\hline & & & & & & & U-series (C-4) & $133 \pm 6$ & & \\
\hline & & & & & & & U-series (C-5) & $123 \pm 8$ & & \\
\hline \multirow{4}{*}{800} & \multirow{4}{*}{$\begin{array}{l}40.561826 \\
14.212505\end{array}$} & \multirow{4}{*}{$\begin{array}{l}\text { Grotta Testa del Cavallo } \\
\text { Capri }\end{array}$} & \multirow{4}{*}{ SLI } & \multirow{4}{*}{ NTC } & \multirow{4}{*}{$5.2 \pm 0.1$} & \multirow{4}{*}{$5.2 \pm 0.2$} & U-series (C-1) & $128 \pm 5$ & \multirow{4}{*}{5,2} & \multirow{4}{*}{$\begin{array}{l}\text { Ferranti \& Antonioli, } 2007 \\
\text { Brancaccio et al., } 1978\end{array}$} \\
\hline & & & & & & & U-series (C-3) & $131 \pm 6$ & & \\
\hline & & & & & & & U-series (C-4) & $133 \pm 6$ & & \\
\hline & & & & & & & U-series (C-5) & $123 \pm 8$ & & \\
\hline \multirow{4}{*}{801} & \multirow{4}{*}{$\begin{array}{l}40.553387 \\
14.200844\end{array}$} & & & & & & U-series (C-1) & $128 \pm 5$ & & \\
\hline & & Grotta Jannarella & SII & NTTC & $\mathrm{C}$ & 640 & U-series (C-3) & $131 \pm 6$ & 50 & Ferranti \& Antonioli, 2007 \\
\hline & & Capri & $\mathrm{SLI}$ & NAT & $0.4 \pm 0.1$ & $0.4 \pm 0.2$ & U-series (C-4) & $133 \pm 6$ & $J, 2$ & Brancaccio et al., 1978 \\
\hline & & & & & & & U-series (C-5) & $123 \pm 8$ & & \\
\hline & & & & & & & U-series (C-1) & $128 \pm 5$ & & \\
\hline 802 & 40.548815 & P.ta Campitiello & SUI & NTC & $62+01$ & $62+02$ & U-series (C-3) & $131 \pm 6$ & 52 & Ferranti \& Antonioli, 2007 \\
\hline 002 & 14.197435 & Capri & SLI & NIC & $0.2 \pm 0.1$ & $0.2 \pm 0.2$ & U-series (C-4) & $133 \pm 6$ & $J, 2$ & Brancaccio et al., 1978 \\
\hline & & & & & & & U-series (C-5) & $123 \pm 8$ & & \\
\hline & & & & & & & U-series (C-1) & $128 \pm 5$ & & \\
\hline 803 & 40.541143 & Cala del Limmo & SII & NTC & $73+01$ & $73+02$ & U-series (C-3) & $131 \pm 6$ & 52 & Ferranti \& Antonioli, 2007 \\
\hline 003 & 14.199689 & Capri & SL1 & NIC & $1 . J \pm 0.1$ & $1.0 \pm 0.2$ & U-series (C-4) & $133 \pm 6$ & $J, 2$ & Brancaccio et al., 1978 \\
\hline & & & & & & & U-series (C-5) & $123 \pm 8$ & & \\
\hline & & & & & & & U-series (C-1) & $128 \pm 5$ & & \\
\hline 805 & $\begin{array}{l}40.538602 \\
14.204492\end{array}$ & $\begin{array}{l}\text { Cala Articola (II) } \\
\text { Capri }\end{array}$ & SLI & NTC & $5.7 \pm 0.1$ & $5.7 \pm 0.2$ & U-series (C-3) & $131 \pm 6$ & 5,2 & $\begin{array}{l}\text { Ferranti \& Antonioli, } 2007 \\
\text { Brancaccio et al.. } 1978\end{array}$ \\
\hline & & & & & & & U-series (C-4) & $133 \pm 6$ & & \\
\hline
\end{tabular}




\begin{tabular}{|c|c|c|c|c|c|c|c|c|c|c|}
\hline $\begin{array}{l}\text { WALIS } \\
\text { ID }\end{array}$ & $\begin{array}{l}\text { Latitude } \\
\text { Longitude }\end{array}$ & Site & $\begin{array}{l}\text { Type of } \\
\text { datapoint }\end{array}$ & $\begin{array}{l}\text { RSL } \\
\text { Indicator }\end{array}$ & $\begin{array}{l}\text { Elevation } \\
(\mathrm{m})\end{array}$ & $\begin{array}{l}\text { Paleo RSL } \\
\text { (m) }\end{array}$ & Dating (Original ID) & $\begin{array}{l}\text { Age (ka or } \\
\text { MIS) }\end{array}$ & $\begin{array}{l}\text { Quality } \\
\text { (RSL, } \\
\text { Age) }\end{array}$ & Reference(s) \\
\hline & & & & & & & U-series (C-5) & $123 \pm 8$ & & \\
\hline \multirow{4}{*}{804} & \multirow{4}{*}{$\begin{array}{l}40.537802 \\
14.204509\end{array}$} & \multirow{4}{*}{$\begin{array}{l}\text { Cala Articola (I) } \\
\text { Capri }\end{array}$} & \multirow{4}{*}{ SLI } & \multirow{4}{*}{ NTC } & \multirow{4}{*}{$6.3 \pm 0.1$} & \multirow{4}{*}{$6.3 \pm 0.2$} & U-series (C-1) & $128 \pm 5$ & \multirow{4}{*}{5,2} & \multirow{4}{*}{$\begin{array}{l}\text { Ferranti \& Antonioli, } 2007 \\
\text { Brancaccio et al., } 1978\end{array}$} \\
\hline & & & & & & & U-series (C-3) & $131 \pm 6$ & & \\
\hline & & & & & & & U-series (C-4) & $133 \pm 6$ & & \\
\hline & & & & & & & U-series (C-5) & $123 \pm 8$ & & \\
\hline \multirow{4}{*}{806} & \multirow{4}{*}{$\begin{array}{l}40.538289 \\
14.205436\end{array}$} & \multirow{4}{*}{$\begin{array}{l}\text { Cala Articola (III) } \\
\text { Capri }\end{array}$} & \multirow{4}{*}{ SLI } & \multirow{4}{*}{ NTC } & \multirow{4}{*}{$7 \pm 0.1$} & \multirow{4}{*}{$7 \pm 0.2$} & U-series (C-1) & $128 \pm 5$ & \multirow{4}{*}{5,2} & \multirow{4}{*}{$\begin{array}{l}\text { Ferranti \& Antonioli, } 2007 \\
\text { Brancaccio et al., } 1978\end{array}$} \\
\hline & & & & & & & U-series (C-3) & $131 \pm 6$ & & \\
\hline & & & & & & & U-series (C-4) & $133 \pm 6$ & & \\
\hline & & & & & & & U-series (C-5) & $123 \pm 8$ & & \\
\hline \multirow{4}{*}{807} & \multirow{4}{*}{$\begin{array}{l}40.540234 \\
14.214724\end{array}$} & \multirow{4}{*}{$\begin{array}{l}\text { Grotta Verde (I) } \\
\text { Capri }\end{array}$} & \multirow{4}{*}{ SLI } & \multirow{4}{*}{ NTC } & \multirow{4}{*}{$6.9 \pm 0.1$} & \multirow{4}{*}{$6.9 \pm 0.2$} & U-series (C-1) & $128 \pm 5$ & \multirow{4}{*}{5,2} & \multirow{4}{*}{$\begin{array}{l}\text { Ferranti \& Antonioli, } 2007 \\
\text { Brancaccio et al., } 1978\end{array}$} \\
\hline & & & & & & & U-series (C-3) & $131 \pm 6$ & & \\
\hline & & & & & & & U-series (C-4) & $133 \pm 6$ & & \\
\hline & & & & & & & U-series (C-5) & $123 \pm 8$ & & \\
\hline \multirow{4}{*}{808} & \multirow{4}{*}{$\begin{array}{l}40.539815 \\
14.220159\end{array}$} & & & & & & U-series (C-1) & $128 \pm 5$ & & \\
\hline & & Grotta Verde (II) & SIJ & NTC & $68+01$ & $68+02$ & U-series (C-3) & $131 \pm 6$ & 52 & Ferranti \& Antonioli, 2007 \\
\hline & & Capri & $\mathrm{SLA}$ & 1010 & 0.0 .0 .1 & $0.0+0.2$ & U-series (C-4) & $133 \pm 6$ & $J, 2$ & Brancaccio et al., 1978 \\
\hline & & & & & & & U-series (C-5) & $123 \pm 8$ & & \\
\hline & & & & & & & U-series (C-1) & $128 \pm 5$ & & \\
\hline 800 & 40.544828 & Gr. Belvedere & SII & NTC & $7+01$ & $7+0 ?$ & U-series (C-3) & $131 \pm 6$ & 52 & Ferranti \& Antonioli, 2007 \\
\hline $00 \%$ & 14.248626 & Capri & $\mathrm{SLI}$ & 1010 & 1.0 .1 & $1 \pm 0.2$ & U-series (C-4) & $133 \pm 6$ & $J_{2}$ & Brancaccio et al., 1978 \\
\hline & & & & & & & U-series (C-5) & $123 \pm 8$ & & \\
\hline & & & & & & & U-series (C-1) & $128 \pm 5$ & & \\
\hline 810 & 40.544077 & Gr. Fontolina & SII & NTC & $72+01$ & $72+02$ & U-series (C-3) & $131 \pm 6$ & 52 & Ferranti \& Antonioli, 2007 \\
\hline 010 & 14.249734 & Capri & SLI & 1010 & $1.2 \mathrm{NV} .1$ & $1.2 \mathrm{LV} .2$ & U-series (C-4) & $133 \pm 6$ & $J, 2$ & Brancaccio et al., 1978 \\
\hline & & & & & & & U-series (C-5) & $123 \pm 8$ & & \\
\hline & & & & & & & U-series (C-1) & $128 \pm 5$ & & \\
\hline 811 & 40.540329 & I Faraglioni & SII & NTC & $7+01$ & $7+02$ & U-series (C-3) & $131 \pm 6$ & 52 & Ferranti \& Antonioli, 2007 \\
\hline 011 & 14.253427 & Capri & $\mathrm{SLI}$ & 1010 & ID.1 & $1+0.2$ & U-series (C-4) & $133 \pm 6$ & $J, 2$ & Brancaccio et al., 1978 \\
\hline & & & & & & & U-series (C-5) & $123 \pm 8$ & & \\
\hline
\end{tabular}




\begin{tabular}{|c|c|c|c|c|c|c|c|c|c|c|}
\hline $\begin{array}{l}\text { WALIS } \\
\text { ID }\end{array}$ & $\begin{array}{l}\text { Latitude } \\
\text { Longitude }\end{array}$ & Site & $\begin{array}{l}\text { Type of } \\
\text { datapoint }\end{array}$ & $\begin{array}{l}\text { RSL } \\
\text { Indicator }\end{array}$ & $\begin{array}{l}\text { Elevation } \\
(\mathbf{m})\end{array}$ & $\begin{array}{l}\text { Paleo RSL } \\
\text { (m) }\end{array}$ & Dating (Original ID) & $\begin{array}{l}\text { Age (ka or } \\
\text { MIS) }\end{array}$ & $\begin{array}{l}\text { Quality } \\
\text { (RSL, } \\
\text { Age) }\end{array}$ & Reference(s) \\
\hline \multirow{4}{*}{812} & \multirow{4}{*}{$\begin{array}{l}40.544389 \\
14.253885\end{array}$} & \multirow{4}{*}{$\begin{array}{l}\text { Porto di Tragara (I) } \\
\text { Capri }\end{array}$} & \multirow{4}{*}{ SLI } & \multirow{4}{*}{ NTC } & \multirow{4}{*}{$8 \pm 0.1$} & \multirow{4}{*}{$8 \pm 0.2$} & U-series (C-1) & $128 \pm 5$ & \multirow{4}{*}{5,2} & \multirow{4}{*}{$\begin{array}{l}\text { Ferranti \& Antonioli, } 2007 \\
\text { Brancaccio et al., } 1978\end{array}$} \\
\hline & & & & & & & U-series (C-3) & $131 \pm 6$ & & \\
\hline & & & & & & & U-series (C-4) & $133 \pm 6$ & & \\
\hline & & & & & & & U-series (C-5) & $123 \pm 8$ & & \\
\hline \multirow{4}{*}{813} & \multirow{4}{*}{$\begin{array}{l}40.545543 \\
14.25671\end{array}$} & \multirow{4}{*}{$\begin{array}{l}\text { Porto di Tragara (II) } \\
\text { Capri }\end{array}$} & \multirow{4}{*}{ SLI } & \multirow{4}{*}{ NTC } & \multirow{4}{*}{$7.9 \pm 0.1$} & \multirow{4}{*}{$7.9 \pm 0.2$} & U-series (C-1) & $128 \pm 5$ & \multirow{4}{*}{5,2} & \multirow{4}{*}{$\begin{array}{l}\text { Ferranti \& Antonioli, } 2007 \\
\text { Brancaccio et al., } 1978\end{array}$} \\
\hline & & & & & & & U-series (C-3) & $131 \pm 6$ & & \\
\hline & & & & & & & U-series (C-4) & $133 \pm 6$ & & \\
\hline & & & & & & & U-series (C-5) & $123 \pm 8$ & & \\
\hline \multirow{4}{*}{814} & \multirow{4}{*}{$\begin{array}{l}40.548585 \\
14.258138\end{array}$} & \multirow{4}{*}{$\begin{array}{l}\text { P.ta di Masullo } \\
\text { Capri }\end{array}$} & \multirow{4}{*}{ SLI } & \multirow{4}{*}{ NTC } & \multirow{4}{*}{$7.1 \pm 0.1$} & \multirow{4}{*}{$7.1 \pm 0.2$} & U-series (C-1) & $128 \pm 5$ & \multirow{4}{*}{5,2} & \multirow{4}{*}{$\begin{array}{l}\text { Ferranti \& Antonioli, } 2007 \\
\text { Brancaccio et al., } 1978\end{array}$} \\
\hline & & & & & & & U-series (C-3) & $131 \pm 6$ & & \\
\hline & & & & & & & U-series (C-4) & $133 \pm 6$ & & \\
\hline & & & & & & & U-series (C-5) & $123 \pm 8$ & & \\
\hline \multirow{4}{*}{815} & \multirow{4}{*}{$\begin{array}{l}40.55045 \\
14.258592\end{array}$} & & & & & & U-series (C-1) & $128 \pm 5$ & & \\
\hline & & a vascio funno & SUI & NTC & $74+01$ & $74+02$ & U-series (C-3) & $131 \pm 6$ & 52 & Ferranti \& Antonioli, 2007 \\
\hline & & Capri & $\mathrm{SLI}$ & NTe & $1.4 \pm 0.1$ & $1.4 \pm 0.2$ & U-series (C-4) & $133 \pm 6$ & $J, 2$ & Brancaccio et al., 1978 \\
\hline & & & & & & & U-series (C-5) & $123 \pm 8$ & & \\
\hline & & & & & & & U-series (C-1) & $128 \pm 5$ & & \\
\hline 816 & 40.551218 & Cala Matermiana & SUI & NTC & $73+01$ & $73+02$ & U-series (C-3) & $131 \pm 6$ & & Ferranti \& Antonioli, 2007 \\
\hline 810 & 14.259126 & Capri & SLI & NIC & $1.5 \pm 0.1$ & $7.3 \pm 0.2$ & U-series (C-4) & $133 \pm 6$ & 5,2 & Brancaccio et al., 1978 \\
\hline & & & & & & & U-series (C-5) & $123 \pm 8$ & & \\
\hline & & & & & & & U-series (C-1) & $128 \pm 5$ & & \\
\hline 817 & 40.552457 & Gr. Bianca (I) & SU & NTC & $73+01$ & $73+02$ & U-series (C-3) & $131 \pm 6$ & 52 & Ferranti \& Antonioli, 2007 \\
\hline 011 & 14.260347 & Capri & SLI & NOC & $1.5 \pm 0.1$ & $1.5 \pm 0.2$ & U-series (C-4) & $133 \pm 6$ & $J, 2$ & Brancaccio et al., 1978 \\
\hline & & & & & & & U-series (C-5) & $123 \pm 8$ & & \\
\hline & & & & & & & U-series (C-1) & $128 \pm 5$ & & \\
\hline & 40.552671 & Gr. Bianca (II) & & & & & U-series (C-3) & $131 \pm 6$ & & Ferranti \& Antonioli, 2007 \\
\hline 818 & 14.260601 & Capri & SLI & NTC & $7.5 \pm 0.1$ & $7.5 \pm 0.2$ & U-series (C-4) & $133 \pm 6$ & 5,2 & Brancaccio et al., 1978 \\
\hline & & & & & & & U-series (C-5) & $123 \pm 8$ & & \\
\hline 819 & & & SLI & NTC & $7.5 \pm 0.1$ & $7.5 \pm 0.2$ & U-series (C-1) & $128 \pm 5$ & 5,2 & \\
\hline
\end{tabular}




\begin{tabular}{|c|c|c|c|c|c|c|c|c|c|c|}
\hline $\begin{array}{l}\text { WALIS } \\
\text { ID }\end{array}$ & $\begin{array}{l}\text { Latitude } \\
\text { Longitude }\end{array}$ & Site & $\begin{array}{l}\text { Type of } \\
\text { datapoint }\end{array}$ & $\begin{array}{l}\text { RSL } \\
\text { Indicator }\end{array}$ & $\begin{array}{l}\text { Elevation } \\
(\mathbf{m})\end{array}$ & $\begin{array}{l}\text { Paleo RSL } \\
\text { (m) }\end{array}$ & Dating (Original ID) & $\begin{array}{l}\text { Age (ka or } \\
\text { MIS) }\end{array}$ & $\begin{array}{l}\text { Quality } \\
\text { (RSL, } \\
\text { Age) }\end{array}$ & Reference(s) \\
\hline & & & & & & & U-series (C-3) & $131 \pm 6$ & & \\
\hline & $\begin{array}{l}40.553863 \\
14.262962\end{array}$ & $\begin{array}{l}\text { P.ta della Chiavica } \\
\text { Capri }\end{array}$ & & & & & U-series (C-4) & $133 \pm 6$ & & $\begin{array}{l}\text { Ferranti \& Antonioli, } 2007 \\
\text { Brancaccio et al.. } 1978\end{array}$ \\
\hline & & & & & & & U-series (C-5) & $123 \pm 8$ & & \\
\hline \multirow{4}{*}{820} & \multirow{4}{*}{$\begin{array}{l}40.554617 \\
14.265368\end{array}$} & \multirow{4}{*}{$\begin{array}{l}\text { P.ta del Monaco } \\
\text { Capri }\end{array}$} & \multirow{4}{*}{ SLI } & \multirow{4}{*}{ NTC } & \multirow{4}{*}{$7.7 \pm 0.1$} & \multirow{4}{*}{$7.7 \pm 0.2$} & U-series (C-1) & $128 \pm 5$ & \multirow{4}{*}{5,2} & \multirow{4}{*}{$\begin{array}{l}\text { Ferranti \& Antonioli, } 2007 \\
\text { Brancaccio et al., } 1978\end{array}$} \\
\hline & & & & & & & U-series (C-3) & $131 \pm 6$ & & \\
\hline & & & & & & & U-series (C-4) & $133 \pm 6$ & & \\
\hline & & & & & & & U-series (C-5) & $123 \pm 8$ & & \\
\hline \multirow{3}{*}{721} & \multirow{3}{*}{$\begin{array}{l}40.574093 \\
14.339977\end{array}$} & \multirow{3}{*}{ Cala di Ieranto } & \multirow{3}{*}{ SLI } & \multirow{3}{*}{ NTC } & \multirow{3}{*}{$7.5 \pm 0.5$} & \multirow{3}{*}{$7.5 \pm 0.5$} & U-series (C-3) & $131 \pm 6$ & \multirow{3}{*}{5,2} & \multirow{3}{*}{ Brancaccio et al., 1978} \\
\hline & & & & & & & U-series (C-4) & $133 \pm 6$ & & \\
\hline & & & & & & & U-series (C-5) & $123 \pm 8$ & & \\
\hline \multirow{4}{*}{823} & \multirow{4}{*}{$\begin{array}{l}40.613132 \\
14.520381\end{array}$} & \multirow{4}{*}{ Vettica Maggiore } & \multirow{4}{*}{ SLI } & \multirow{4}{*}{ NTC } & \multirow{4}{*}{$6.8 \pm 0.1$} & \multirow{4}{*}{$6.8 \pm 0.2$} & U-series (C-1) & $128 \pm 5$ & \multirow{4}{*}{5,2} & \multirow{4}{*}{$\begin{array}{l}\text { Ferranti \& Antonioli, } 2007 \\
\text { Brancaccio et al., } 1978\end{array}$} \\
\hline & & & & & & & U-series (C-3) & $131 \pm 6$ & & \\
\hline & & & & & & & U-series (C-4) & $133 \pm 6$ & & \\
\hline & & & & & & & U-series (C-5) & $123 \pm 8$ & & \\
\hline 798 & $\begin{array}{l}40.614439 \\
14.567143 \\
\end{array}$ & Grotta dello Smeraldo & SLI & LIT & $8 \pm 1.6$ & $7.8 \pm 1.9$ & $\begin{array}{l}\text { STRAT ( } P \text {. latus or } \\
\text { Senegalese fauna) }\end{array}$ & MIS 5 & 4,3 & $\begin{array}{l}\text { Cosentino \& Gliozzi, } 1988 \\
\text { Colantoni, } 1970\end{array}$ \\
\hline 767 & $\begin{array}{l}40.613522 \\
14.575294\end{array}$ & $\begin{array}{l}\text { Conca dei Marini (III) } \\
\text { Site I }\end{array}$ & SLI & NTC & $3.5 \pm 0.5$ & $3.5 \pm 0.5$ & U-series (CM6) & $<109.1 \pm 7.3$ & 5,2 & $\begin{array}{l}\text { Iannace et al., } 2003 \\
\text { Brancaccio et al., } 1978 \\
\text { Riccio et al., } 2001\end{array}$ \\
\hline \multirow{2}{*}{766} & \multirow{2}{*}{$\begin{array}{l}40.613522 \\
14.575294\end{array}$} & \multirow{2}{*}{$\begin{array}{l}\text { Conca dei Marini (II) } \\
\text { Site I }\end{array}$} & SUI & NTC & $65+05$ & $65+05$ & U-series (C-1) & $128 \pm 5$ & 52 & Brancaccinet al 1978 \\
\hline & & & SLI & NIC & $0.5 \pm 0.5$ & $0.5 \pm 0.5$ & U-series* (C-2) & $87 \pm 10$ & 5,2 & Brancaccio et al., $19 / 8$ \\
\hline 720 & 40.613522 & Conca dei Marini (I) & SLI & NTC & $7.5 \pm 0.5$ & $7.5 \pm 0.5$ & U-series (C-1) & $128 \pm 5$ & 52 & Brancaccin et al 1978 \\
\hline 120 & 14.575294 & Site I & SLI & NTE & $1.5 \pm 0.5$ & $1.5 \pm 0.5$ & U-series* (C-2) & $87 \pm 10$ & $J, 2$ & Drancactio et al., 1970 \\
\hline 3623 & 40.613522 & Conca dei Marini (V) & SLI & NTC & $2 \pm 0.5$ & $2 \pm 0.5$ & U-series (C-1) & $<128 \pm 5$ & 5,2 & Riccio et al., 2001 \\
\hline 5023 & 14.575294 & Conca der Mrarmin (v) & SLI & NIC & $2 \pm 0.5$ & $2 \pm 0.5$ & U-series* (C-2) & $<87 \pm 10$ & 5,2 & Brancaccio et al., 1978 \\
\hline 3565 & 40.613522 & Conca dei Marini (IV) & SLI & NTC & $8+16$ & $8+16$ & U-series (C-1) & $128 \pm 5$ & 33 & $\begin{array}{l}\text { Iannace et al., } 2003 \\
\text { Brancaccio et }\end{array}$ \\
\hline 3565 & 14.575294 & Site I & $\mathrm{SL1}$ & 1010 & 0.1 .0 & $0 \pm 1.0$ & U-series* (C-2) & $87 \pm 10$ & 3,3 & $\begin{array}{l}\text { Brancaccio et al., } 19 / 8 \\
\text { Riccio et al., } 2001\end{array}$ \\
\hline 769 & $\begin{array}{l}40.495303 \\
15.017081 \\
\end{array}$ & Ponte Barizzo & MLI & & $25 \pm 2.5$ & & AAR (N/A) & $130 \pm 39$ & 0,3 & $\begin{array}{l}\text { Brancaccio et al.,1986 } \\
\text { Brancaccio et al., } 1987 \\
\end{array}$ \\
\hline 770 & $\begin{array}{l}40.472432 \\
14.994638 \\
\end{array}$ & Gromola & MLI & & $13 \pm 1.3$ & & AAR (N/A) & $100 \pm 30$ & 0,3 & $\begin{array}{l}\text { Brancaccio et al.,1986 } \\
\text { Brancaccio et al., } 1987\end{array}$ \\
\hline 728 & $\begin{array}{l}40.245714 \\
14.908187 \\
\end{array}$ & Licosa Cape (III) & SLI & MT & $9.5 \pm 1.9$ & $9.4 \pm 2.1$ & U-series (Canna-2) & $>102 \pm 4$ & 4,3 & $\begin{array}{l}\text { Iannace et al., } 2001 \\
\text { Cinque et al., } 1994\end{array}$ \\
\hline
\end{tabular}




\begin{tabular}{|c|c|c|c|c|c|c|c|c|c|c|}
\hline $\begin{array}{l}\text { WALIS } \\
\text { ID }\end{array}$ & $\begin{array}{l}\text { Latitude } \\
\text { Longitude }\end{array}$ & Site & $\begin{array}{l}\text { Type of } \\
\text { datapoint }\end{array}$ & $\begin{array}{l}\text { RSL } \\
\text { Indicator }\end{array}$ & $\begin{array}{l}\text { Elevation } \\
\text { (m) }\end{array}$ & $\begin{array}{l}\text { Paleo RSL } \\
\text { (m) }\end{array}$ & Dating (Original ID) & $\begin{array}{l}\text { Age (ka or } \\
\text { MIS) }\end{array}$ & $\begin{array}{l}\text { Quality } \\
\text { (RSL, } \\
\text { Age) }\end{array}$ & Reference(s) \\
\hline 727 & $\begin{array}{l}40.245714 \\
14.908187\end{array}$ & Licosa Cape (II) & SLI & MT & $1.5 \pm 0.3$ & $1.4 \pm 1$ & U-series (Canna-2) & $<102 \pm 4$ & 5,3 & $\begin{array}{l}\text { Iannace et al., } 2001 \\
\text { Cinque et al., } 1994\end{array}$ \\
\hline 726 & $\begin{array}{l}40.245714 \\
14.908187\end{array}$ & Licosa Cape (I) & SLI & MT & $5 \pm 1$ & $4.9 \pm 1.4$ & U-series (Canna-2) & $102 \pm 4$ & 4,3 & $\begin{array}{l}\text { Iannace et al., } 2001 \\
\text { Cinque et al., } 1994\end{array}$ \\
\hline 592 & $\begin{array}{l}40.235994 \\
14.960996 \\
\end{array}$ & $\begin{array}{l}\text { Ogliastro Marina } \\
\text { Baia Arena }\end{array}$ & SLI & MT & $7 \pm 1.4$ & $6.9 \pm 1.7$ & $\begin{array}{l}\text { AAR (Ogliasto Marina } \\
\text { (Baia Arena) }\end{array}$ & $110 \pm 33$ & 4,3 & Brancaccio et al., 1990 \\
\hline 593 & $\begin{array}{l}40.036429 \\
15.285242\end{array}$ & $\begin{array}{l}\text { Palinuro } \\
\text { Lido Ficocella }\end{array}$ & MLI & & $8 \pm 1.6$ & $0 \pm 1.6$ & $\begin{array}{l}\text { AAR (Palinuro (Lido } \\
\text { Ficocella) }\end{array}$ & $110 \pm 33$ & 0,3 & Brancaccio et al., 1990 \\
\hline \multirow{2}{*}{732} & \multirow{2}{*}{$\begin{array}{l}40.024122 \\
15.283719\end{array}$} & \multirow{2}{*}{ Palinuro Cape } & \multirow{2}{*}{ SLI } & \multirow{2}{*}{ NTC } & \multirow{2}{*}{$2.2 \pm 0.4$} & \multirow{2}{*}{$2.2 \pm 0.4$} & U-series (N/A) & $\begin{array}{l}<165+55- \\
36\end{array}$ & \multirow{2}{*}{5,4} & \multirow{2}{*}{$\begin{array}{l}\text { Antonioli et al., 1994a } \\
\text { Antonioli et al., 1994b }\end{array}$} \\
\hline & & & & & & & $\begin{array}{l}\text { STRAT (P. latus or } \\
\text { Senegalese fauna) }\end{array}$ & MIS 5 & & \\
\hline \multirow{3}{*}{3568} & \multirow{3}{*}{$\begin{array}{l}40.000312 \\
15.426755\end{array}$} & \multirow{3}{*}{$\begin{array}{l}\text { The Riparo Infreschi } \\
\text { cave } \\
\text { (I) }\end{array}$} & \multirow{3}{*}{ SLI } & \multirow{3}{*}{ LIT } & \multirow{3}{*}{$8.9 \pm 0.6$} & \multirow{3}{*}{$8.8 \pm 0.6$} & $\begin{array}{l}\text { U-series (UMD180823- } \\
362 \text { ) }\end{array}$ & $120.8 \pm 1.1$ & \multirow{3}{*}{5,5} & \multirow{3}{*}{$\begin{array}{l}\text { Bini et al., } 2020 \\
\text { Esposito et al.,2003 }\end{array}$} \\
\hline & & & & & & & $\begin{array}{l}\text { U-series (UME190501- } \\
\text { 284) }\end{array}$ & $120.4 \pm 1$ & & \\
\hline & & & & & & & $\begin{array}{l}\text { U-series (UME190812- } \\
343 \text { ) }\end{array}$ & $120.2 \pm 1.7$ & & \\
\hline 3569 & $\begin{array}{l}40.000312 \\
15.426755\end{array}$ & $\begin{array}{l}\text { The Riparo Infreschi } \\
\text { cave } \\
\text { (II) }\end{array}$ & TLI & & $4.5 \pm 0.1$ & $4.4 \pm 0.2$ & $\begin{array}{l}\text { U-series (UMD180823- } \\
340)\end{array}$ & $200 \pm 2.1$ & 0,5 & Bini et al., 2020 \\
\hline 3570 & $\begin{array}{l}40.000312 \\
15.426755\end{array}$ & $\begin{array}{l}\text { The Riparo Infreschi } \\
\text { cave } \\
\text { (III) }\end{array}$ & TLI & & $4.5 \pm 0.1$ & $4.4 \pm 0.2$ & $\begin{array}{l}\text { U-series (UMD180823- } \\
344 \text { ) }\end{array}$ & $206.2 \pm 3.2$ & 0,5 & Bini et al., 2020 \\
\hline 3571 & $\begin{array}{l}40.000312 \\
15.426755\end{array}$ & $\begin{array}{l}\text { The Riparo Infreschi } \\
\text { cave } \\
\text { (IV) }\end{array}$ & TLI & & $4.1 \pm 0.1$ & $4 \pm 0.2$ & $\begin{array}{l}\text { U-series (UMD180823- } \\
345 \text { ) }\end{array}$ & $214.2 \pm 2.1$ & 0,5 & Bini et al., 2020 \\
\hline 3572 & $\begin{array}{l}40.000312 \\
15.426755\end{array}$ & $\begin{array}{l}\text { The Riparo Infreschi } \\
\text { cave } \\
\text { (V) }\end{array}$ & TLI & & $4.1 \pm 0.1$ & $4 \pm 0.2$ & $\begin{array}{l}\text { U-series (UMD180823- } \\
\text { 349) }\end{array}$ & $239.5 \pm 3.1$ & 0,5 & Bini et al., 2020 \\
\hline 3573 & $\begin{array}{l}40.000312 \\
15.426755\end{array}$ & $\begin{array}{l}\text { The Riparo Infreschi } \\
\text { cave } \\
\text { (VI) }\end{array}$ & TLI & & $3.7 \pm 0.1$ & $3.6 \pm 0.2$ & $\begin{array}{l}\text { U-series (UMD180823- } \\
362 \text { ) }\end{array}$ & $120.8 \pm 1.1$ & 0,5 & Bini et al., 2020 \\
\hline 3574 & $\begin{array}{l}40.000312 \\
15.426755\end{array}$ & $\begin{array}{l}\text { The Riparo Infreschi } \\
\text { cave } \\
\text { (VII) }\end{array}$ & TLI & & $3.7 \pm 0.1$ & $3.6 \pm 0.2$ & $\begin{array}{l}\text { U-series (UME190501- } \\
\text { 284) }\end{array}$ & $120.4 \pm 1$ & 0,5 & Bini et al., 2020 \\
\hline 3575 & $\begin{array}{l}40.000312 \\
15.426755\end{array}$ & $\begin{array}{l}\text { The Riparo Infreschi } \\
\text { cave } \\
\text { (VIII) }\end{array}$ & TLI & & $3.8 \pm 0.1$ & $3.7 \pm 0.2$ & $\begin{array}{l}\text { U-series (UME190812- } \\
343 \text { ) }\end{array}$ & $120.2 \pm 1.7$ & 0,5 & Bini et al., 2020 \\
\hline 3576 & $\begin{array}{l}40.000312 \\
15.426755\end{array}$ & $\begin{array}{l}\text { The Riparo Infreschi } \\
\text { cave } \\
\text { (IX) }\end{array}$ & TLI & & $2.7 \pm 0.1$ & $2.6 \pm 0.2$ & $\begin{array}{l}\text { STRAT (Tephra in } \\
\text { aeolian sand) }\end{array}$ & MIS 5e & 0,5 & Bini et al., 2020 \\
\hline 792 & $\begin{array}{l}40.069976 \\
15.616391 \\
\end{array}$ & Sapri (II) & SLI & MT & $8 \pm 1.6$ & $7.8 \pm 1.9$ & AAR (Sapri (Limito) & $110 \pm 33$ & 4,3 & Brancaccio et al., 1990 \\
\hline 791 & $\begin{array}{l}40.07138 \\
15.617157\end{array}$ & Sapri & SLI & MT & $15 \pm 3$ & $14.8 \pm 3.2$ & AAR (Sapri (Limito) & $>110 \pm 33$ & 3,3 & Brancaccio et al., 1990 \\
\hline
\end{tabular}




\begin{tabular}{|c|c|c|c|c|c|c|c|c|c|c|}
\hline $\begin{array}{l}\text { WALIS } \\
\text { ID }\end{array}$ & $\begin{array}{l}\text { Latitude } \\
\text { Longitude }\end{array}$ & Site & $\begin{array}{l}\text { Type of } \\
\text { datapoint }\end{array}$ & $\begin{array}{l}\text { RSL } \\
\text { Indicator }\end{array}$ & $\begin{array}{l}\text { Elevation } \\
\text { (m) }\end{array}$ & $\begin{array}{l}\text { Paleo RSL } \\
\text { (m) }\end{array}$ & Dating (Original ID) & $\begin{array}{l}\text { Age (ka or } \\
\text { MIS) }\end{array}$ & $\begin{array}{l}\text { Quality } \\
\text { (RSL, } \\
\text { Age) }\end{array}$ & Reference(s) \\
\hline \multicolumn{11}{|c|}{ Italy (Calabria) } \\
\hline \multirow{9}{*}{267} & \multirow{9}{*}{$\begin{array}{l}38.713512 \\
16.119394\end{array}$} & \multirow{9}{*}{ Vibo Marina } & \multirow{9}{*}{ SLI } & \multirow{9}{*}{ MT } & \multirow{9}{*}{$50 \pm 5$} & \multirow{9}{*}{$49.8 \pm 5.1$} & U-series (A) & $122.4 \pm 36.7$ & \multirow{9}{*}{2,3} & \multirow{9}{*}{$\begin{array}{l}\text { Bianca et al., } 2011 \\
\text { Dai Pra et al., } 1993 \\
\text { Pata, } 1947 \\
\text { Roberts et a., } 2013 \\
\text { Balescu et al., } 1997 \\
\text { Dumas et al., } 1991\end{array}$} \\
\hline & & & & & & & U-series (B) & $119 \pm 35.7$ & & \\
\hline & & & & & & & U-series (VV1w) & $132 \pm 1.6$ & & \\
\hline & & & & & & & U-series (VV1s) & $142 \pm 1.8$ & & \\
\hline & & & & & & & U-series (N/A) & $130 \pm 8$ & & \\
\hline & & & & & & & AAR (Vibo Marina) & MIS 5e & & \\
\hline & & & & & & & LUM (TB) & $94 \pm 8$ & & \\
\hline & & & & & & & LUM (VM21) & $192 \pm 19$ & & \\
\hline & & & & & & & $\begin{array}{l}\text { STRAT (P. latus or } \\
\text { Senegalese fauna) }\end{array}$ & MIS 5 & & \\
\hline \multirow{3}{*}{315} & \multirow{3}{*}{$\begin{array}{l}38.706274 \\
16.046311\end{array}$} & \multirow{3}{*}{ Paradisoni } & \multirow{3}{*}{ SLI } & \multirow{3}{*}{ MT } & \multirow{3}{*}{$140 \pm 0.2$} & \multirow{3}{*}{$139.8 \pm 0.9$} & AAR (Paradisoni) & MIS 5e & \multirow{3}{*}{4,4} & \multirow{3}{*}{ Balescu et al., 1997} \\
\hline & & & & & & & LUM (PA21) & $128 \pm 13$ & & \\
\hline & & & & & & & LUM (PA21) & $104 \pm 8$ & & \\
\hline 316 & $\begin{array}{l}38.710179 \\
16.036594\end{array}$ & Sacco & SLI & MT & $153 \pm 15.3$ & $152.8 \pm 15.3$ & LUM (SC21) & $134 \pm 13$ & 2,2 & Balescu et al., 1997 \\
\hline 268 & $\begin{array}{l}38.724333 \\
16.0333\end{array}$ & Briatico & SLI & MT & $100 \pm 10$ & $99.9 \pm 10$ & LUM (TB) & $94 \pm 8$ & 2,2 & Bianca et al., 2011 \\
\hline 269 & $\begin{array}{l}38.61922 \\
15.829895 \\
\end{array}$ & Capo Vaticano & SLI & MT & $175 \pm 17.4$ & $174.9 \pm 17.4$ & LUM (TB) & $94 \pm 8$ & 2,2 & Bianca et al., 2011 \\
\hline 270 & $\begin{array}{l}38.552123 \\
15.930741\end{array}$ & Nicotera & SLI & MT & $125 \pm 1$ & $124.9 \pm 1.3$ & LUM (TB) & $94 \pm 8$ & 2,2 & Bianca et al., 2011 \\
\hline 3431 & $\begin{array}{l}38.55 \\
15.94 \\
\end{array}$ & $\begin{array}{l}\text { Gioia Tauro } \\
\text { Contrada Iannì (nearby } \\
\text { Nicotera) }\end{array}$ & SLI & MT & $80 \pm 8$ & $79.9 \pm 8$ & $\begin{array}{l}\text { STRAT ( } P . \text { latus or } \\
\text { Senegalese fauna) }\end{array}$ & MIS 5 & 2,3 & $\begin{array}{l}\text { Bonfiglio et al., } 1988 \\
\text { Miyauchi et al., } 1994\end{array}$ \\
\hline 3430 & $\begin{array}{l}38.529562 \\
16.040374\end{array}$ & Gioia Tauro basin & SLI & MT & $65 \pm 6.5$ & $64.9 \pm 6.5$ & $\begin{array}{l}\text { STRAT (P. latus or } \\
\text { Senegalese fauna) }\end{array}$ & MIS 5 & 2,3 & Cosentino \& Gliozzi, 1988 \\
\hline 314 & $\begin{array}{l}38.481234 \\
15.956639 \\
\end{array}$ & Rosarno & SLI & MT & $80 \pm 8$ & $79.9 \pm 8$ & LUM (RO21) & $140 \pm 13$ & 2,2 & Balescu et al., 1997 \\
\hline \multirow{6}{*}{304} & \multirow{6}{*}{$\begin{array}{l}38.078356 \\
15.639365\end{array}$} & \multirow{6}{*}{ Ravagnese } & \multirow{6}{*}{ SLI } & \multirow{6}{*}{ MT } & \multirow{6}{*}{$129 \pm 12.9$} & \multirow{6}{*}{$128.9 \pm 12.9$} & AAR (Ravagnese) & MIS 5e & \multirow{6}{*}{3,5} & \multirow{6}{*}{$\begin{array}{l}\text { Balescu et al., } 1997 \\
\text { Dumas et al., } 1988 \\
\text { Gignoux, } 1913\end{array}$} \\
\hline & & & & & & & AAR (Ravagnese) & MIS 5e & & \\
\hline & & & & & & & AAR (RSH2) & MIS 5e & & \\
\hline & & & & & & & AAR (RSH2) & MIS 5e & & \\
\hline & & & & & & & AAR (RSH2) & MIS 5e & & \\
\hline & & & & & & & AAR (RA3) & MIS 5e & & \\
\hline
\end{tabular}




\begin{tabular}{|c|c|c|c|c|c|c|c|c|c|c|}
\hline $\begin{array}{l}\text { WALIS } \\
\text { ID }\end{array}$ & $\begin{array}{l}\text { Latitude } \\
\text { Longitude }\end{array}$ & Site & $\begin{array}{l}\text { Type of } \\
\text { datapoint }\end{array}$ & $\begin{array}{l}\text { RSL } \\
\text { Indicator }\end{array}$ & $\begin{array}{l}\text { Elevation } \\
\text { (m) }\end{array}$ & $\begin{array}{l}\text { Paleo RSL } \\
\text { (m) }\end{array}$ & Dating (Original ID) & $\begin{array}{l}\text { Age (ka or } \\
\text { MIS) }\end{array}$ & $\begin{array}{l}\text { Quality } \\
\text { (RSL, } \\
\text { Age) }\end{array}$ & Reference(s) \\
\hline & & & & & & & AAR (RA3) & MIS 5e & & \\
\hline & & & & & & & AAR (RA4) & MIS 5e & & \\
\hline & & & & & & & AAR (RA2) & MIS 5e & & \\
\hline & & & & & & & AAR (RA2) & MIS 5e & & \\
\hline & & & & & & & AAR (CA1) & MIS 5e & & \\
\hline & & & & & & & LUM (RA21) & $116 \pm 13$ & & \\
\hline & & & & & & & LUM (RA22) & $101 \pm 12$ & & \\
\hline & & & & & & & LUM (RA21) & $102 \pm 8$ & & \\
\hline & & & & & & & $\begin{array}{l}\text { STRAT (P. latus or } \\
\text { Senegalese fauna) }\end{array}$ & MIS 5 & & \\
\hline \multirow{3}{*}{312} & \multirow{3}{*}{$\begin{array}{l}38.066454 \\
15.659182\end{array}$} & \multirow{3}{*}{ Trombaca } & \multirow{3}{*}{ SLI } & \multirow{3}{*}{ MT } & \multirow{3}{*}{$116 \pm 11.6$} & \multirow{3}{*}{$115.9 \pm 11.6$} & AAR (Trombaca) & MIS 5e & 2,3 & Balescu et al., 1997 \\
\hline & & & & & & & LUM (TR21) & $79 \pm 8$ & 2,3 & \\
\hline & & & & & & & LUM (TR21) & $90 \pm 9$ & 2,3 & \\
\hline \multirow{14}{*}{306} & \multirow{14}{*}{$\begin{array}{l}38.058666 \\
15.661343\end{array}$} & \multirow{14}{*}{ Bovetto } & \multirow{14}{*}{ SLI } & \multirow{14}{*}{ MT } & \multirow{14}{*}{$129 \pm 12.9$} & \multirow{14}{*}{$128.9 \pm 12.9$} & AAR (Bovetto) & MIS 5e & \multirow{14}{*}{3,5} & \multirow{14}{*}{$\begin{array}{l}\text { Balescu et al., } 1997 \\
\text { Gignoux, } 1913 \\
\text { Dumas et al., } 1988\end{array}$} \\
\hline & & & & & & & AAR (Bovetto) & MIS 5e & & \\
\hline & & & & & & & AAR (RSHx) & MIS 5e & & \\
\hline & & & & & & & AAR (RSHz) & MIS 5e & & \\
\hline & & & & & & & AAR (RSHz) & MIS 5e & & \\
\hline & & & & & & & AAR (RSHz) & MIS 5e & & \\
\hline & & & & & & & AAR (RSH1b) & MIS 5e & & \\
\hline & & & & & & & AAR (RSH1b) & MIS 5e & & \\
\hline & & & & & & & AAR (RSH1b) & MIS 5e & & \\
\hline & & & & & & & AAR (RSH1a) & MIS 5e & & \\
\hline & & & & & & & AAR (RSH1c) & MIS 5e & & \\
\hline & & & & & & & AAR (RSH1d) & MIS 5e & & \\
\hline & & & & & & & LUM (BO21) & $116 \pm 12$ & & \\
\hline & & & & & & & $\begin{array}{l}\text { STRAT ( } P \text {. latus or } \\
\text { Senegalese fauna) }\end{array}$ & MIS 5 & & \\
\hline 4065 & $\begin{array}{l}38.063325 \\
15.675959 \\
\end{array}$ & Trombaca (II) & SLI & MT & $100 \pm 10$ & $99.9 \pm 10$ & AAR (TR1) & MIS 5c & 2,3 & Dumas et al., 1988 \\
\hline 4066 & & Traietta & SLI & MT & $83 \pm 8.3$ & $82.9 \pm 8.3$ & AAR (RA1) & MIS 5a & 2,2 & Dumas et al., 1988 \\
\hline
\end{tabular}




\begin{tabular}{|c|c|c|c|c|c|c|c|c|c|c|}
\hline $\begin{array}{l}\text { WALIS } \\
\text { ID }\end{array}$ & $\begin{array}{l}\text { Latitude } \\
\text { Longitude }\end{array}$ & Site & $\begin{array}{l}\text { Type of } \\
\text { datapoint }\end{array}$ & $\begin{array}{l}\text { RSL } \\
\text { Indicator }\end{array}$ & $\begin{array}{l}\text { Elevation } \\
\text { (m) }\end{array}$ & $\begin{array}{l}\text { Paleo RSL } \\
\text { (m) }\end{array}$ & Dating (Original ID) & $\begin{array}{l}\text { Age (ka or } \\
\text { MIS) }\end{array}$ & $\begin{array}{l}\text { Quality } \\
\text { (RSL, } \\
\text { Age) }\end{array}$ & Reference(s) \\
\hline & $\begin{array}{l}38.066238 \\
15.67265\end{array}$ & & & & & & AAR (RA1) & MIS 5a & & \\
\hline 4067 & $\begin{array}{l}38.057435 \\
15.681235 \\
\end{array}$ & Rione Branca & SLI & MT & $76 \pm 7.6$ & $75.9 \pm 7.6$ & AAR (RB1) & MIS 5a & 2,2 & Dumas et al., 1988 \\
\hline \multirow{3}{*}{1378} & \multirow{3}{*}{$\begin{array}{l}38.04588 \\
15.678569\end{array}$} & \multirow{3}{*}{$\begin{array}{l}\text { Nocella } \\
\text { Pellaro }\end{array}$} & \multirow{3}{*}{ SLI } & \multirow{3}{*}{ MT } & \multirow{3}{*}{$166 \pm 16.6$} & \multirow{3}{*}{$165.9 \pm 16.6$} & AAR (Nocella) & MIS 5e & \multirow{3}{*}{2,2} & \multirow{3}{*}{$\begin{array}{l}\text { Dumas et al., } 1988 \\
\text { Dumas } 1987 \\
\text { Balescu et al., } 1997\end{array}$} \\
\hline & & & & & & & AAR (Nocella) & MIS 7 & & \\
\hline & & & & & & & $\begin{array}{l}\text { STRAT (P. latus or } \\
\text { Senegalese fauna) }\end{array}$ & MIS 5 & & \\
\hline 4062 & $\begin{array}{l}38.00714 \\
15.64512\end{array}$ & $\begin{array}{l}\text { Nocella (II) } \\
\text { Pellaro }\end{array}$ & SLI & MT & $105 \pm 10.5$ & $104.9 \pm 10.5$ & $\begin{array}{l}\text { STRAT ( } P \text {. latus or } \\
\text { Senegalese fauna) }\end{array}$ & MIS 5 & 2,2 & $\begin{array}{l}\text { Dumas et al., } 1988 \\
\text { Dumas } 1987\end{array}$ \\
\hline \multirow{3}{*}{313} & \multirow{3}{*}{$\begin{array}{l}37.956035 \\
15.683487\end{array}$} & \multirow{3}{*}{ Altibano } & \multirow{3}{*}{ SLI } & \multirow{3}{*}{ MT } & \multirow{3}{*}{$198 \pm 0.2$} & \multirow{3}{*}{$197.9 \pm 0.7$} & AAR (Altibano) & MIS 7 & \multirow{3}{*}{4,2} & \multirow{3}{*}{$\begin{array}{l}\text { Balescu et al., } 1997 \\
\text { Dumas et al., } 1988\end{array}$} \\
\hline & & & & & & & $\operatorname{AAR}(\mathrm{AB} 1)$ & MIS 5e & & \\
\hline & & & & & & & LUM (AB21) & $118 \pm 13$ & & \\
\hline \multirow{2}{*}{563} & \multirow{2}{*}{$\begin{array}{l}38.952446 \\
16.983983\end{array}$} & \multirow{2}{*}{ San Leonardo di Cutro } & \multirow{2}{*}{ SLI } & \multirow{2}{*}{ MT } & \multirow{2}{*}{$83 \pm 8.3$} & \multirow{2}{*}{$82.9 \pm 8.3$} & LUM (SldC 1) & $121 \pm 15$ & \multirow{2}{*}{2,3} & \multirow{2}{*}{ Mauz and Hassler, 2000} \\
\hline & & & & & & & LUM (SldC 1) & $142 \pm 20$ & & \\
\hline 4068 & $\begin{array}{l}38.926101 \\
17.006657 \\
\end{array}$ & Campolongo & SLI & MT & $90 \pm 9$ & $89.9 \pm 9$ & AAR (Campolongo 32) & $110 \pm 33$ & 2,3 & $\begin{array}{l}\text { Belluomini et al., } 1988 \\
\text { Nalin et al., } 2020\end{array}$ \\
\hline 4069 & $\begin{array}{l}38.913225 \\
17.029234\end{array}$ & Le Castella & SLI & MT & $90 \pm 9$ & $89.9 \pm 9$ & AAR (Le Castella 45) & $110 \pm 33$ & 2,3 & $\begin{array}{l}\text { Belluomini et al., } 1988 \\
\text { Nalin et al., 2020 }\end{array}$ \\
\hline \multirow{3}{*}{566} & \multirow{3}{*}{$\begin{array}{l}39.030593 \\
17.000421\end{array}$} & \multirow{3}{*}{ Manca della Vozza } & & & & & $\begin{array}{l}\text { AAR (Manca della } \\
\text { Vozza 13) }\end{array}$ & MIS 7 & & \\
\hline & & & SLI & MT & $240 \pm 24$ & $239.1 \pm 24$ & LUM (MdV) & $>90 \pm 10$ & 2,3 & $\begin{array}{l}\text { Mauz and Hassler, } 2000 \\
\text { Belluomini et al., } 1988\end{array}$ \\
\hline & & & & & & & LUM (MdV) & $>85 \pm 10$ & & \\
\hline & & & & & & & $\begin{array}{l}\text { AAR (Isola Capo } \\
\text { Rizzuto 8) }\end{array}$ & $<110 \pm 33$ & & \\
\hline 565 & 38.956174 & Isola di Capo Rizzuto & SLI & MT & $81 \pm 8.1$ & $80.9 \pm 8.2$ & LUM (IdCR05) & $>98 \pm 7$ & 2,2 & Mauz and Hassler, 2000 \\
\hline & & & & & & & LUM (IdCR03) & $>82 \pm 12$ & & \\
\hline & & & & & & & LUM (IdCR03) & $>149 \pm 64$ & & \\
\hline & & & & & & & AAR (Capo Rizzuto 1c) & $110 \pm 33$ & & \\
\hline 564 & & $C=\mathrm{C}$ & CII & MT & $10+26$ & 170.27 & AAR (Capo Rizzuto 9) & $110 \pm 33$ & 2 & Mauz and Hassler, 2000 \\
\hline 564 & 17.094202 & Capo Rizzuto & SLI & MT & $18 \pm 3.6$ & $17.9 \pm 3.7$ & LUM (CR2) & $86 \pm 9$ & 2,3 & $\begin{array}{l}\text { Belluomını et al., } 1988 \\
\text { Gliozzi } 1987\end{array}$ \\
\hline & & & & & & & LUM (CR2) & $65 \pm 6$ & & \\
\hline 3428 & $\begin{array}{l}38.959999 \\
17.139995 \\
\end{array}$ & Crotone (II) & SLI & MT & $110 \pm 11$ & $109.9 \pm 11$ & $\begin{array}{l}\text { STRAT ( } P . \text { latus or } \\
\text { Senegalese fauna) }\end{array}$ & MIS 5 & 2,3 & Cosentino \& Gliozzi, 1988 \\
\hline 1379 & $\begin{array}{l}38.959958 \\
17.14032 \\
\end{array}$ & Crotone & SLI & MT & $84 \pm 8.4$ & $83.9 \pm 8.5$ & $\begin{array}{l}\text { STRAT (P. latus or } \\
\text { Senegalese fauna) }\end{array}$ & MIS 5 & 2,3 & Palmentola et al., 1990 \\
\hline
\end{tabular}




\begin{tabular}{|c|c|c|c|c|c|c|c|c|c|c|}
\hline $\begin{array}{l}\text { WALIS } \\
\text { ID }\end{array}$ & $\begin{array}{l}\text { Latitude } \\
\text { Longitude }\end{array}$ & Site & $\begin{array}{l}\text { Type of } \\
\text { datapoint }\end{array}$ & $\begin{array}{l}\text { RSL } \\
\text { Indicator }\end{array}$ & $\begin{array}{l}\text { Elevation } \\
(\mathbf{m})\end{array}$ & $\begin{array}{l}\text { Paleo RSL } \\
\text { (m) }\end{array}$ & Dating (Original ID) & $\begin{array}{l}\text { Age (ka or } \\
\text { MIS) }\end{array}$ & $\begin{array}{l}\text { Quality } \\
\text { (RSL, } \\
\text { Age) }\end{array}$ & Reference(s) \\
\hline \multirow[b]{2}{*}{3634} & \multirow{2}{*}{$\begin{array}{l}39.020459 \\
17.175222\end{array}$} & \multirow[b]{2}{*}{ Capo Colonna Terrace } & \multirow[b]{2}{*}{ SLI } & \multirow[b]{2}{*}{ MT } & \multirow[b]{2}{*}{$35 \pm 7$} & \multirow[b]{2}{*}{$34.9 \pm 7.1$} & AAR (Capo Colonne 3) & $70 \pm 21$ & \multirow[b]{2}{*}{1,2} & \multirow{2}{*}{$\begin{array}{l}\text { Nalin et al., } 2012 \\
\text { Belluomini et al., } 1988 \\
\text { Palmentola et al., } 1990\end{array}$} \\
\hline & & & & & & & $\begin{array}{l}\text { STRAT (P. latus or } \\
\text { Senegalese fauna) }\end{array}$ & MIS 5 & & \\
\hline 442 & $\begin{array}{l}39.611016 \\
16.445736 \\
\end{array}$ & Vaccarizzo & SLI & MT & $125 \pm 12.5$ & $124.9 \pm 12.5$ & ESR (K-5355) & $135 \pm 20$ & 3,2 & Santoro et al., 2009 \\
\hline \multirow{2}{*}{324} & \multirow{2}{*}{$\begin{array}{l}39.871248 \\
16.523172\end{array}$} & \multirow{2}{*}{ Trebisacce } & \multirow{2}{*}{ SLI } & \multirow{2}{*}{ MT } & \multirow{2}{*}{$128 \pm 12.8$} & \multirow{2}{*}{$127.9 \pm 12.8$} & $\mathrm{AAR}^{*}(1)$ & $139 \pm 41.7$ & \multirow{2}{*}{3,3} & \multirow{2}{*}{ Cucci, 2004} \\
\hline & & & & & & & AAR (2) & $130 \pm 39$ & & \\
\hline \multicolumn{11}{|c|}{ Italy (Basilicata) } \\
\hline 416 & $\begin{array}{l}40.139879 \\
16.651067 \\
\end{array}$ & Piano San Nicola & SLI & MT & $90 \pm 9$ & $90 \pm 9$ & AAR (Site No 1) & MIS 5a & 2,2 & $\begin{array}{l}\text { Hearty and Dai Pra, } 1992 \text { Journal of } \\
\text { Coastal Research }\end{array}$ \\
\hline \multirow{2}{*}{4061} & \multirow{2}{*}{$\begin{array}{l}40.32933 \\
16.747113\end{array}$} & \multirow{2}{*}{$\begin{array}{l}\text { Metaponto (ii) } \\
\text { San Teodoro I }\end{array}$} & \multirow{2}{*}{ SLI } & \multirow{2}{*}{ MT } & \multirow{2}{*}{$42 \pm 6.4$} & \multirow{2}{*}{$41.9 \pm 6.4$} & U-series (BR80-001) & $>75 \pm 3.3$ & \multirow{2}{*}{4,2} & \multirow{2}{*}{$\begin{array}{l}\text { Brückner, } 1980 \\
\text { Sauer et al., } 2010 \\
\text { Zander et al., } 2006\end{array}$} \\
\hline & & & & & & & U-series (BR80-002) & $>110 \pm 10$ & & \\
\hline \multirow{2}{*}{4060} & & Metaponto & SLI & MT & $22+2.4$ & & U-series (BR80-001) & $75 \pm 7$ & 42 & $\begin{array}{l}\text { Brückner, } 1980 \\
\text { Sauer et } 2010\end{array}$ \\
\hline & 16.777243 & Petrulla & SLI & M1 & $22 \pm 2.4$ & $21.9 \pm 2.5$ & U-series (BR80-002) & $110 \pm 10$ & 4,2 & $\begin{array}{l}\text { Sauer et al., } 2010 \\
\text { Zander et al., } 2006\end{array}$ \\
\hline Italy (Pu & & & & & & & & & & \\
\hline & & & & & & & AAR (Site No 2) & MIS 5e & & \\
\hline & & & & & & & AAR (Site No 2) & MIS 5e & & Boenzi et al., 1985 \\
\hline 355 & 16.938602 & Ponte del Re & SLI & MT & $45 \pm 4.5$ & $44.3 \pm 4.5$ & AAR (Site No 2) & MIS 5e & 2,3 & $\begin{array}{l}\text { Dai Pra \& Hearty, } 1988 \\
\text { Hearty and Dai Pra, } 1992\end{array}$ \\
\hline & & & & & & & $\begin{array}{l}\text { STRAT ( } P . \text { latus or } \\
\text { Senegalese fauna) }\end{array}$ & MIS 5 & & \\
\hline & & & & & & & U-series (PR1 [6468]) & $107.7 \pm 1.5$ & & \\
\hline 3631 & 40.52951 & Ponte Romano & SLI & MT & $37 \pm 2$ & $36.9 \pm 2.1$ & LUM (N/A) & $93 \pm 8$ & 4,3 & Mastronuzzi \& Sansò, 2003 \\
\hline & & Taranto & & & & & $\begin{array}{l}\text { STRAT ( } P . \text { latus or } \\
\text { Senegalese fauna) }\end{array}$ & MIS 5 & & Mastronuzzi \& Sansò, 2002 \\
\hline 697 & $\begin{array}{l}40.531944 \\
17.183889 \\
\end{array}$ & $\begin{array}{l}\text { Masseria S. Giovanni } \\
\text { Taranto }\end{array}$ & SLI & MT & $32.5 \pm 6.5$ & $32.4 \pm 6.5$ & U-series (LJ402) & $142 \pm 14$ & 2,3 & Dai Pra and Stearns, 1977 \\
\hline & & & & & & & U-series (PR) & MIS 5a & & \\
\hline 224 & $\begin{array}{l}40.478337 \\
17.179617\end{array}$ & Punta Rondinella & MLI & & $1.5 \pm 0.2$ & & $\mathrm{AAR}(\mathrm{PR})$ & MIS 5a & 0,1 & Belluomini et al., 2002 \\
\hline & & & & & & & AAR (PR) & MIS 3 & & \\
\hline & & & & & & & U-series (ISP2) & $86.4 \pm 4.2$ & & \\
\hline & & & & & & & U-series (ISP2) & MIS 3 & & \\
\hline 225 & $\begin{array}{l}40.457552 \\
17.161104\end{array}$ & Punta lo Scanno & SLI & BRK & $2 \pm 0.2$ & $2.1 \pm 0.5$ & AAR (ISP2) & MIS 5a & 3,1 & Belluomini et al., 2002 \\
\hline & & & & & & & AAR (ISP2) & MIS 3 & & \\
\hline & & & & & & & AAR (ISP2) & MIS 3 & & \\
\hline
\end{tabular}




\begin{tabular}{|c|c|c|c|c|c|c|c|c|c|c|}
\hline $\begin{array}{l}\text { WALIS } \\
\text { ID }\end{array}$ & $\begin{array}{l}\text { Latitude } \\
\text { Longitude }\end{array}$ & Site & $\begin{array}{l}\text { Type of } \\
\text { datapoint }\end{array}$ & $\begin{array}{l}\text { RSL } \\
\text { Indicator }\end{array}$ & $\begin{array}{l}\text { Elevation } \\
(\mathbf{m})\end{array}$ & $\begin{array}{l}\text { Paleo RSL } \\
\text { (m) }\end{array}$ & Dating (Original ID) & $\begin{array}{l}\text { Age (ka or } \\
\text { MIS) }\end{array}$ & $\begin{array}{l}\text { Quality } \\
\text { (RSL, } \\
\text { Age) }\end{array}$ & Reference(s) \\
\hline 228 & $\begin{array}{l}40.453135 \\
17.157284 \\
\end{array}$ & Apodonia & MLI & & $0.6 \pm 0.2$ & & U-series (ISP4) & MIS 5e & 0,2 & Belluomini et al., 2002 \\
\hline 3632 & $\begin{array}{l}40.484115 \\
17.220128 \\
\end{array}$ & $\begin{array}{l}\text { Masseria Ruggiero } \\
\text { Taranto }\end{array}$ & MLI & & $18 \pm 3.6$ & & $\begin{array}{l}\text { STRAT (P. latus or } \\
\text { Senegalese fauna) }\end{array}$ & MIS 5 & 0,3 & Mastronuzzi \& Sansò, 2003 \\
\hline \multirow{3}{*}{704} & \multirow{3}{*}{$\begin{array}{l}40.497194 \\
17.239483\end{array}$} & \multirow{3}{*}{ Masseria Bagnara } & \multirow{3}{*}{ MLI } & & \multirow{3}{*}{$14 \pm 2.8$} & & U-series (N/A) & $134 \pm 16$ & \multirow{3}{*}{0,4} & \multirow{3}{*}{ Dai Pra \& Hearty, 1988} \\
\hline & & & & & & & AAR (N/A) & MIS 5e & & \\
\hline & & & & & & & AAR (N/A) & MIS 5e & & \\
\hline 223 & $\begin{array}{l}40.501098 \\
17.24893 \\
\end{array}$ & $\begin{array}{l}\text { Masseria Santa } \\
\text { Teresiola }\end{array}$ & SLI & BRK & $9.3 \pm 0.3$ & $9.3 \pm 0.6$ & U-series (MP7) & $89.8 \pm 4.8$ & 4,3 & $\begin{array}{l}\text { Belluomini et al., } 2002 \\
\text { Dai Pra \& Hearty, } 1988\end{array}$ \\
\hline \multirow{3}{*}{3630} & \multirow{3}{*}{$\begin{array}{l}40.501098 \\
17.24893\end{array}$} & \multirow{3}{*}{$\begin{array}{l}\text { Masseria Santa } \\
\text { Teresiola (II) }\end{array}$} & \multirow{3}{*}{ SLI } & \multirow{3}{*}{ BRK } & \multirow{3}{*}{$28 \pm 5.6$} & \multirow{3}{*}{$28 \pm 5.6$} & U-series (N/A) & $162 \pm 48.6$ & \multirow{3}{*}{2,4} & \multirow{3}{*}{$\begin{array}{l}\text { Dai Pra \& Hearty, } 1988 \\
\text { Cotecchia et al., } 1969 \\
\text { Dai Pra and Stearns, } 1977 \\
\text { Hearty and Dai Pra, } 1992 \\
\text { Mastronuzzi \& Sansò, } 2003\end{array}$} \\
\hline & & & & & & & U-series (N/A) & $125 \pm 8$ & & \\
\hline & & & & & & & $\begin{array}{l}\text { STRAT ( } P . \text { latus or } \\
\text { Senegalese fauna) }\end{array}$ & MIS 5 & & \\
\hline \multirow{2}{*}{699} & \multirow{2}{*}{$\begin{array}{l}40.50924 \\
17.317213\end{array}$} & \multirow{2}{*}{ Masseria San Pietro } & \multirow{2}{*}{ SLI } & \multirow{2}{*}{ MT } & \multirow{2}{*}{$28 \pm 5.6$} & \multirow{2}{*}{$27.9 \pm 5.6$} & $\mathrm{AAR}(\mathrm{MII} \mathrm{Sb})$ & MIS 5e & \multirow{2}{*}{2,2} & \multirow{2}{*}{$\begin{array}{l}\text { Dai Pra and Stearns, } 1977 \\
\text { Hearty and Dai Pra, } 1992 \\
\text { Hearty et al., } 1985\end{array}$} \\
\hline & & & & & & & $\begin{array}{l}\text { STRAT (P. latus or } \\
\text { Senegalese fauna) }\end{array}$ & MIS 5 & & \\
\hline \multirow{17}{*}{230} & \multirow{17}{*}{$\begin{array}{l}40.473951 \\
17.310684\end{array}$} & \multirow{17}{*}{ Il Fronte } & \multirow{17}{*}{ MLI } & & & & U-series (FW11-C1-A) & $130.6 \pm 1.2$ & & \\
\hline & & & & & & & U-series (FW11-C1-B) & $134.8 \pm 0.9$ & & \\
\hline & & & & & & & U-series (FW11-C1-c) & $139.9 \pm 2.9$ & & \\
\hline & & & & & & & U-series (FW11-C1-D) & $138 \pm 3.1$ & & \\
\hline & & & & & & & U-series (FW11-C1-E) & $131.5 \pm 2.4$ & & \\
\hline & & & & & & & U-series (FW11-C1-F) & $146.9 \pm 3.4$ & & \\
\hline & & & & & & & U-series (FW11-C2-A) & $123.7 \pm 1.4$ & & \\
\hline & & & & & & & U-series (FW11-C2-B) & $125.1 \pm 0.8$ & & Amorosi et al., 2014 \\
\hline & & & & & $7.5 \pm 0.5$ & & U-series (FW11-C2-C) & $121 \pm 2.1$ & 0,4 & $\begin{array}{l}\text { Dai Pra \& Hearty, } 1988 \\
\text { Dai Pra and Stearns, } 1977\end{array}$ \\
\hline & & & & & & & U-series (FW11-C2-D) & $124.9 \pm 2.7$ & & Hearty et al., 1986 \\
\hline & & & & & & & U-series (LJ483) & $87 \pm 4$ & & \\
\hline & & & & & & & U-series (LJ481) & $106 \pm 8$ & & \\
\hline & & & & & & & U-series (LJ505) & $130 \pm 10$ & & \\
\hline & & & & & & & U-series (LJ487) & $154 \pm 13$ & & \\
\hline & & & & & & & U-series (TSh5b2) & $117 \pm 7$ & & \\
\hline & & & & & & & U-series (TSh5b2) & $128 \pm 7$ & & \\
\hline & & & & & & & U-series (TSh5a) & $121 \pm 6$ & & \\
\hline
\end{tabular}




\begin{tabular}{|c|c|c|c|c|c|c|c|c|c|c|}
\hline $\begin{array}{l}\text { WALIS } \\
\text { ID }\end{array}$ & $\begin{array}{l}\text { Latitude } \\
\text { Longitude }\end{array}$ & Site & $\begin{array}{l}\text { Type of } \\
\text { datapoint }\end{array}$ & $\begin{array}{l}\text { RSL } \\
\text { Indicator }\end{array}$ & $\begin{array}{l}\text { Elevation } \\
(\mathbf{m})\end{array}$ & $\begin{array}{l}\text { Paleo RSL } \\
\text { (m) }\end{array}$ & Dating (Original ID) & $\begin{array}{l}\text { Age (ka or } \\
\text { MIS) }\end{array}$ & $\begin{array}{l}\text { Quality } \\
\text { (RSL, } \\
\text { Age) }\end{array}$ & Reference(s) \\
\hline & & & & & & & AAR (N/A) & MIS 5e & & \\
\hline & & & & & & & $\begin{array}{l}\text { STRAT (P. latus or } \\
\text { Senegalese fauna) }\end{array}$ & MIS 5 & & \\
\hline \multirow{2}{*}{701} & \multirow{2}{*}{$\begin{array}{l}40.471535 \\
17.30819\end{array}$} & \multirow{2}{*}{ Masseria Pantaleo } & \multirow{2}{*}{ MLI } & & \multirow{2}{*}{$11 \pm 2.2$} & & U-series (LJ438) & $142 \pm 14$ & \multirow{2}{*}{0,3} & \multirow{2}{*}{ Dai Pra and Stearns, 1977} \\
\hline & & & & & & & U-series (LJ439) & $146 \pm 12$ & & \\
\hline \multirow{4}{*}{698} & \multirow{4}{*}{$\begin{array}{l}40.399548 \\
17.252443\end{array}$} & \multirow{4}{*}{$\begin{array}{l}\text { Carelli } \\
\text { Between Carelli and } \\
\text { Saturo harbour }\end{array}$} & \multirow{4}{*}{ SLI } & \multirow{4}{*}{ MT } & \multirow{4}{*}{$20 \pm 4$} & \multirow{4}{*}{$19.9 \pm 4.1$} & U-series (LJ425) & $138 \pm 14$ & \multirow{4}{*}{2,4} & \multirow{4}{*}{$\begin{array}{l}\text { Dai Pra and Stearns, } 1977 \\
\text { Hearty and Dai Pra, } 1992 \text { Journal of } \\
\text { Coastal Research } \\
\text { Hearty et al., } 1985\end{array}$} \\
\hline & & & & & & & AAR (MIV) & $<$ MIS 5 & & \\
\hline & & & & & & & AAR (MIV) & $<$ MIS 5 & & \\
\hline & & & & & & & $\begin{array}{l}\text { STRAT (P. latus or } \\
\text { Senegalese fauna) }\end{array}$ & MIS 5 & & \\
\hline \multirow{6}{*}{700} & \multirow{6}{*}{$\begin{array}{l}40.399548 \\
17.252443\end{array}$} & \multirow{6}{*}{$\begin{array}{l}\text { Il Posto } \\
\text { Between Il Posto and } \\
\text { Torre Castelluccia }\end{array}$} & \multirow{6}{*}{ SLI } & \multirow{6}{*}{ MT } & \multirow{6}{*}{$29 \pm 5.8$} & \multirow{6}{*}{$28.9 \pm 5.8$} & U-series (SV16) & $139.6 \pm 4.5$ & \multirow{6}{*}{2,4} & \multirow{6}{*}{$\begin{array}{l}\text { Dai Pra and Stearns, } 1977 \\
\text { Belluomini et al., } 2002 \\
\text { Hearty and Dai Pra, } 1992 \\
\text { Hearty et al., } 1985\end{array}$} \\
\hline & & & & & & & U-series (LJ425) & $138 \pm 14$ & & \\
\hline & & & & & & & AAR (SV16) & MIS 5e & & \\
\hline & & & & & & & AAR (SV2) & MIS 5c & & \\
\hline & & & & & & & AAR (MIII) & MIS 5e & & \\
\hline & & & & & & & $\begin{array}{l}\text { STRAT (P. latus or } \\
\text { Senegalese fauna) }\end{array}$ & MIS 5 & & \\
\hline 226 & $\begin{array}{l}40.361735 \\
17.325993 \\
\end{array}$ & Punta della Baracca & SLI & BRK & $3.5 \pm 0.2$ & $3.6 \pm 0.5$ & U-series (SV16) & $139.6 \pm 4.5$ & 3,4 & Belluomini et al., 2002 \\
\hline 705 & $\begin{array}{l}40.342522 \\
17.380951 \\
\end{array}$ & Torre Castelluccia & SLI & BRK & $3 \pm 0.6$ & $3.2 \pm 0.8$ & AAR (N/A) & MIS 5c & 4,3 & Dai Pra \& Hearty, 1988 \\
\hline 695 & $\begin{array}{l}40.375273 \\
17.428416 \\
\end{array}$ & Lizzano & SLI & MT & $28 \pm 5.6$ & $27.9 \pm 5.7$ & $\begin{array}{l}\text { STRAT (P. latus or } \\
\text { Senegalese fauna) }\end{array}$ & MIS 5 & 2,2 & Dai Pra and Stearns, 1977 \\
\hline \multirow[b]{2}{*}{696} & \multirow{2}{*}{$\begin{array}{l}40.287224 \\
17.819483\end{array}$} & \multirow[b]{2}{*}{ Torre Castiglione } & \multirow[b]{2}{*}{ SLI } & \multirow[b]{2}{*}{ MT } & & & U-series (LJ423) & $156 \pm 20$ & & \\
\hline & & & & & $2.5 \pm 0.5$ & $2.4 \pm 1$ & $\begin{array}{l}\text { STRAT ( } P . \text { latus or } \\
\text { Senegalese fauna) }\end{array}$ & MIS 5 & 4,1 & Dai Pra and Stearns, 1977 \\
\hline & & & & & & & U-series (N/A) & $115 \pm 11$ & & Cotecchia et al., 1969 \\
\hline 3625 & $\begin{array}{l}40.07983 \\
18007961\end{array}$ & Torre Sabea & SLI & BRK & $3 \pm 0.6$ & $3 \pm 0.6$ & AAR (MIII Sb) & MIS 5e & 4,4 & Dai Pra \& Hearty, 1988 \\
\hline & $18.00 / 901$ & Gallipoli & & & & & $\begin{array}{l}\text { STRAT (P. latus or } \\
\text { Senegalese fauna) }\end{array}$ & MIS 5 & & Hearty et al., 1985 \\
\hline & & & & & & & AAR (MIIIb Sb) & MIS 5e & & Cotecchia et al., 1969 \\
\hline & & & & & & & AAR (MIIIa) & MIS 5e & & Dai Pra \& Hearty, 1988 \\
\hline 3621 & 17.995678 & Gallipoll & SLI & ВКК & $10 \pm 2$ & $10 \pm 2$ & AAR (MIII) & MIS 5e & 4,3 & Hearty et al., 1985 \\
\hline & & & & & & & AAR (MIIIa) & MIS 5e & & Mastronuzzi \& Sansò, 2003 \\
\hline
\end{tabular}




\begin{tabular}{|c|c|c|c|c|c|c|c|c|c|c|}
\hline $\begin{array}{l}\text { WALIS } \\
\text { ID }\end{array}$ & $\begin{array}{l}\text { Latitude } \\
\text { Longitude }\end{array}$ & Site & $\begin{array}{l}\text { Type of } \\
\text { datapoint }\end{array}$ & $\begin{array}{l}\text { RSL } \\
\text { Indicator }\end{array}$ & $\begin{array}{l}\text { Elevation } \\
(\mathbf{m})\end{array}$ & $\begin{array}{l}\text { Paleo RSL } \\
\text { (m) }\end{array}$ & Dating (Original ID) & $\begin{array}{l}\text { Age (ka or } \\
\text { MIS) }\end{array}$ & $\begin{array}{l}\text { Quality } \\
\text { (RSL, } \\
\text { Age) }\end{array}$ & Reference(s) \\
\hline & & & & & & & $\begin{array}{l}\text { STRAT (P. latus or } \\
\text { Senegalese fauna) }\end{array}$ & MIS 5 & & \\
\hline \multirow{5}{*}{3628} & \multirow{5}{*}{$\begin{array}{l}40.050975 \\
18.001356\end{array}$} & \multirow{5}{*}{$\begin{array}{l}\text { Torre San Giovanni } \\
\text { Gallipoli }\end{array}$} & \multirow{5}{*}{ SLI } & \multirow{5}{*}{ BRK } & \multirow{5}{*}{$8 \pm 1.6$} & \multirow{5}{*}{$8 \pm 1.6$} & AAR (DI) & $>$ MIS 5a & \multirow{5}{*}{5,3} & \multirow{5}{*}{$\begin{array}{l}\text { Cotecchia et al., } 1969 \\
\text { Dai Pra \& Hearty, } 1988 \\
\text { Hearty and Dai Pra, } 1992 \\
\text { Hearty et al., } 1985 \\
\text { Mastronuzzi \& Sansò, } 2003\end{array}$} \\
\hline & & & & & & & AAR (SI) & $>$ MIS 5a & & \\
\hline & & & & & & & AAR (SII) & $>$ MIS $5 \mathrm{c}$ & & \\
\hline & & & & & & & AAR (MIII Sb) & $>$ MIS 5 & & \\
\hline & & & & & & & $\begin{array}{l}\text { STRAT (P. latus or } \\
\text { Senegalese fauna) }\end{array}$ & MIS 5 & & \\
\hline 3635 & $\begin{array}{l}39.79143 \\
18.343804 \\
\end{array}$ & Grotta del Diavolo & SLI & BRK & $3 \pm 0.6$ & $3.3 \pm 1$ & U-series (6715) & $>77 \pm 23.1$ & 5,2 & Mastronuzzi et al., 2007 \\
\hline 1324 & $\begin{array}{l}39.855 \\
18.391 \\
\end{array}$ & $\begin{array}{l}\text { Marina di Novaglie } \\
\text { Southern Site }\end{array}$ & SLI & MT & $25 \pm 15$ & $20 \pm 15$ & $\begin{array}{l}\text { STRAT (P. latus or } \\
\text { Senegalese fauna) }\end{array}$ & MIS 5 & 1,2 & $\begin{array}{l}\text { Ferranti et al., } 2006 \\
\text { Parroni \& Silenzi, } 1997\end{array}$ \\
\hline 1323 & $\begin{array}{l}39.862 \\
18.394\end{array}$ & $\begin{array}{l}\text { Marina di Novaglie } \\
\text { Northern Site }\end{array}$ & SLI & MT & $25 \pm 15$ & $20 \pm 15$ & $\begin{array}{l}\text { STRAT (P. latus or } \\
\text { Senegalese fauna) }\end{array}$ & MIS 5 & 1,2 & $\begin{array}{l}\text { Ferranti et al., } 2006 \\
\text { Parroni \& Silenzi, } 1997\end{array}$ \\
\hline 1319 & $\begin{array}{l}40.017 \\
18.434\end{array}$ & Grotta Romanelli & SLI & BRK & $9.8 \pm 0.1$ & $7.8 \pm 0.1$ & $\begin{array}{l}\text { STRAT (P. latus or } \\
\text { Senegalese fauna) }\end{array}$ & MIS 5 & 4,3 & $\begin{array}{l}\text { Cosentino \& Gliozzi, } 1988 \\
\text { Bordoni \& Valensise } 1999 \\
\text { Ferranti et al., 2006 }\end{array}$ \\
\hline 1320 & $\begin{array}{l}40.046433 \\
18.476133 \\
\end{array}$ & Grotta delle Striare & SLI & NTC & $8.2 \pm 0.3$ & $7.2 \pm 0.3$ & $\begin{array}{l}\text { STRAT (P. latus or } \\
\text { Senegalese fauna) }\end{array}$ & MIS 5 & 5,3 & Antonioli et al., 2018 \\
\hline 1321 & $\begin{array}{l}40.717 \\
17.799 \\
\end{array}$ & $\begin{array}{l}\text { Torre Mattarelle - Torre } \\
\text { Guaceto }\end{array}$ & SLI & BRK & $4 \pm 2$ & $2 \pm 2$ & $\begin{array}{l}\text { STRAT ( } P \text {. latus or } \\
\text { Senegalese fauna) }\end{array}$ & MIS 5 & 3,3 & $\begin{array}{l}\text { Ferranti et al., } 2006 \\
\text { Bordoni and Valensise, } 1999\end{array}$ \\
\hline 1322 & $\begin{array}{l}40.76 \\
17.704\end{array}$ & Torre Santa Sabina & SLI & BRK & $3 \pm 2$ & $1 \pm 2$ & $\begin{array}{l}\text { STRAT (P. latus or } \\
\text { Senegalese fauna) }\end{array}$ & MIS 5 & 4,3 & $\begin{array}{l}\text { Ferranti et al., } 2006 \\
\text { Bordoni \& Valensise } 1999\end{array}$ \\
\hline 1318 & $\begin{array}{l}40.815 \\
17.504\end{array}$ & Villanova-Monopoli & MLI & & $30 \pm 3$ & $0 \pm 0$ & $\begin{array}{l}\text { STRAT (P. latus or } \\
\text { Senegalese fauna) }\end{array}$ & MIS 5 & 0,3 & $\begin{array}{l}\text { Antonioli et al., } 2009 \\
\text { Bordoni \& Valensise } 1999 \\
\text { Mastronuzzi et al., } 1989\end{array}$ \\
\hline \multirow{2}{*}{452} & \multirow{2}{*}{$\begin{array}{l}41.506891 \\
15.905839\end{array}$} & \multirow{2}{*}{$\begin{array}{l}\text { MM4 borehole } \\
\text { Unit } 13\end{array}$} & \multirow{2}{*}{ MLI } & & \multirow{2}{*}{$-8 \pm 1.6$} & & AAR (MM4/3) & $70 \pm 19$ & \multirow{2}{*}{0,2} & \multirow{2}{*}{ De Santis et al., 2010} \\
\hline & & & & & & & AAR (MM4/3) & $70 \pm 19$ & & \\
\hline \multirow{2}{*}{453} & \multirow{2}{*}{$\begin{array}{l}41.506891 \\
15.905839\end{array}$} & \multirow{2}{*}{$\begin{array}{l}\text { MM4 borehole } \\
\text { Unit } 11\end{array}$} & \multirow{2}{*}{ MLI } & & \multirow{2}{*}{$-13 \pm 2.6$} & & AAR (MM4/6) & $98 \pm 17$ & \multirow{2}{*}{0,3} & \multirow{2}{*}{ De Santis et al., 2010} \\
\hline & & & & & & & AAR (MM4/6) & $98 \pm 17$ & & \\
\hline 454 & $\begin{array}{l}41.506891 \\
15.905839 \\
\end{array}$ & $\begin{array}{l}\text { MM4 borehole } \\
\text { Unit } 10\end{array}$ & MLI & & $-22 \pm 4.4$ & & STRAT (RPL Unit 10) & MIS 5e & 0,2 & De Santis et al., 2010 \\
\hline 1317 & $\begin{array}{l}41.881 \\
15.287\end{array}$ & $\begin{array}{l}\text { Fortore River } \\
\text { The site is near the } \\
\text { mouth of Fortore River, } \\
\text { near the village of } \\
\text { Ripalta. }\end{array}$ & TLI & & $25 \pm 10$ & & $\begin{array}{l}\text { STRAT (Tyrrhenian } \\
\text { Adriatic (Eemian pollen } \\
\text { unit) }\end{array}$ & MIS 5e & 2,2 & $\begin{array}{l}\text { Bordoni \& Valensise } 1999 \\
\text { Antonioli et al., } 2009\end{array}$ \\
\hline \multicolumn{11}{|c|}{ Italy (Marche) } \\
\hline 1333 & $\begin{array}{l}43.72 \\
13.295\end{array}$ & Metauro River & SLI & MT & $15 \pm 1$ & $12 \pm 1$ & $\begin{array}{l}\text { STRAT (Tyrrhenian } \\
\text { Adriatic (Eemian pollen } \\
\text { unit) }\end{array}$ & MIS 5e & 0,0 & Ferranti et al., 2006 \\
\hline
\end{tabular}




\begin{tabular}{|c|c|c|c|c|c|c|c|c|c|c|}
\hline $\begin{array}{l}\text { WALIS } \\
\text { ID }\end{array}$ & $\begin{array}{l}\text { Latitude } \\
\text { Longitude }\end{array}$ & Site & $\begin{array}{l}\text { Type of } \\
\text { datapoint }\end{array}$ & $\begin{array}{l}\text { RSL } \\
\text { Indicator }\end{array}$ & $\begin{array}{l}\text { Elevation } \\
(\mathbf{m})\end{array}$ & $\begin{array}{l}\text { Paleo RSL } \\
\text { (m) }\end{array}$ & Dating (Original ID) & $\begin{array}{l}\text { Age (ka or } \\
\text { MIS) }\end{array}$ & $\begin{array}{l}\text { Quality } \\
\text { (RSL, } \\
\text { Age) }\end{array}$ & Reference(s) \\
\hline 1332 & $\begin{array}{l}43.78 \\
13.104\end{array}$ & Arzilla River & SLI & MT & $15 \pm 1$ & $12 \pm 1$ & $\begin{array}{l}\text { STRAT (Tyrrhenian } \\
\text { Adriatic (Eemian pollen } \\
\text { unit) }\end{array}$ & MIS 5e & 0,0 & Ferranti et al., 2006 \\
\hline \multicolumn{11}{|c|}{ Italy (Emilia Romagna) } \\
\hline 1331 & $\begin{array}{l}43.982 \\
12.675\end{array}$ & Conca River & SLI & MT & $16 \pm 1$ & $13 \pm 1$ & $\begin{array}{l}\text { STRAT (Tyrrhenian } \\
\text { Adriatic (Eemian pollen } \\
\text { unit) }\end{array}$ & MIS 5e & 0,0 & Ferranti et al., 2006 \\
\hline 1330 & $\begin{array}{l}44.086091 \\
12.536547\end{array}$ & $\begin{array}{l}256-S 3 \text { Viserba } \\
\text { Core } \mathrm{S} 3 \text { of geological } \\
\text { map, sheet } 256 \\
\text { "Rimini", near Viserba; } \\
\text { ground at }+1.5 \mathrm{~m} \text { asl. }\end{array}$ & SLI & BRK & $-78.9 \pm 1$ & $-78.9 \pm 10$ & $\begin{array}{l}\text { STRAT (Tyrrhenian } \\
\text { Adriatic (Eemian pollen } \\
\text { unit) }\end{array}$ & MIS 5e & 3,3 & $\begin{array}{l}\text { Amorosi et al., } 2004 \\
\text { Campo et al., } 2020\end{array}$ \\
\hline 1329 & $\begin{array}{l}44.162311 \\
12.209142\end{array}$ & $\begin{array}{l}255-\mathrm{S} 4 \text { Cesena } \\
\text { Core S4 of geological } \\
\text { map sheet } 255 \\
\text { "Cesena", near Cesena; } \\
\text { ground + } 33.5 \mathrm{~m} \text { asl. }\end{array}$ & TLI & & $-11.5 \pm 1$ & $0 \pm 0$ & $\begin{array}{l}\text { STRAT (Tyrrhenian } \\
\text { Adriatic (Eemian pollen } \\
\text { unit) }\end{array}$ & MIS 5e & 0,3 & $\begin{array}{l}\text { Campo et al., } 2020 \\
\text { Amorosi et al., } 2008\end{array}$ \\
\hline \multirow[b]{2}{*}{1308} & \multirow[b]{2}{*}{$\begin{array}{l}44.287225 \\
12.326817\end{array}$} & \multirow{2}{*}{$\begin{array}{l}\text { 240-S8 Milano } \\
\text { Marittima } \\
\text { Core S8 drilled NW of } \\
\text { the town of Milano } \\
\text { Marittima. Ground } \\
\text { surface at }+1.0 \mathrm{~m} \text { asl. }\end{array}$} & \multirow[b]{2}{*}{ SLI } & \multirow[b]{2}{*}{ BRI } & \multirow[b]{2}{*}{$-117 \pm 1$} & \multirow[b]{2}{*}{$-117 \pm 10$} & ESR (K-4384) & $129 \pm 18$ & \multirow[b]{2}{*}{3,5} & \multirow[b]{2}{*}{$\begin{array}{l}\text { Ferranti et al., } 2006 \\
\text { Amorosi et al., } 2004 \\
\text { Campo et al., } 2020\end{array}$} \\
\hline & & & & & & & $\begin{array}{l}\text { STRAT (Tyrrhenian } \\
\text { Adriatic (Eemian pollen } \\
\text { unit) }\end{array}$ & MIS 5e & & \\
\hline 1328 & $\begin{array}{l}44.306436 \\
12.044187\end{array}$ & $\begin{array}{l}\text { 240-S2 Villafranca } \\
\text { Core S2 of geological } \\
\text { map sheet } 204 \text { "Forlì", } \\
\text { near Villafranca; ground } \\
+13.0 \mathrm{~m} \text { asl. }\end{array}$ & TLI & & $-70 \pm 1$ & $0 \pm 0$ & $\begin{array}{l}\text { STRAT (Tyrrhenian } \\
\text { Adriatic (Eemian pollen } \\
\text { unit) }\end{array}$ & MIS 5e & 0,3 & $\begin{array}{l}\text { Amorosi et al., } 2004 \\
\text { Campo et al., } 2020\end{array}$ \\
\hline 1327 & $\begin{array}{l}44.385075 \\
12.041781\end{array}$ & $\begin{array}{l}\text { 240-S13 Russi } \\
\text { Core S13 of geological } \\
\text { map, sheet } 240 \text { "Forlì", } \\
\text { near Russi, ground } \\
+10.0 \mathrm{~m} \text { asl. } \\
\end{array}$ & SLI & LAG & $-112 \pm 1$ & $-111.5 \pm 1.8$ & $\begin{array}{l}\text { STRAT (Tyrrhenian } \\
\text { Adriatic (Eemian pollen } \\
\text { unit) }\end{array}$ & MIS 5e & 4,4 & $\begin{array}{l}\text { Amorosi et al., } 2004 \\
\text { Campo et al., } 2020\end{array}$ \\
\hline 1325 & $\begin{array}{l}44.414589 \\
12.12844\end{array}$ & $\begin{array}{l}\text { 223-S12 Ravenna } \\
\text { Core S12 of geological } \\
\text { map, sheet } 223 \\
\text { "Ravenna", west of } \\
\text { Ravenna, ground }+1.8 \mathrm{~m} \\
\text { asl. }\end{array}$ & SLI & BRK & $-100 \pm 1$ & $-100 \pm 10$ & $\begin{array}{l}\text { STRAT (Tyrrhenian } \\
\text { Adriatic (Eemian pollen } \\
\text { unit) }\end{array}$ & MIS 5e & 4,3 & $\begin{array}{l}\text { Amorosi et al., } 2014 \\
\text { Campo et al., } 2020\end{array}$ \\
\hline 1326 & $\begin{array}{l}44.56695 \\
12.218758\end{array}$ & $\begin{array}{l}\text { 223-S17 Mandriole } \\
\text { Core S17 of geological } \\
\text { map, sheet } 223 \\
\text { "Ravenna", south of } \\
\text { Valli di Comacchio, } \\
\text { ground } 0.0 \mathrm{~m} \text { asl. }\end{array}$ & SLI & BRK & $-105.5 \pm 1$ & $-105.5 \pm 10$ & $\begin{array}{l}\text { STRAT (Tyrrhenian } \\
\text { Adriatic (Eemian pollen } \\
\text { unit) }\end{array}$ & MIS 5e & 3,5 & $\begin{array}{l}\text { Amorosi et al., } 2014 \\
\text { Campo et al., } 2020\end{array}$ \\
\hline 1310 & & & SLI & BRK & $-85.5 \pm 1$ & $-85.5 \pm 10$ & ESR (K-4385) & $154 \pm 26$ & 3,5 & Ferranti et al., 2006 \\
\hline
\end{tabular}




\begin{tabular}{|c|c|c|c|c|c|c|c|c|c|c|}
\hline $\begin{array}{l}\text { WALIS } \\
\text { ID }\end{array}$ & $\begin{array}{l}\text { Latitude } \\
\text { Longitude }\end{array}$ & Site & $\begin{array}{l}\text { Type of } \\
\text { datapoint }\end{array}$ & $\begin{array}{l}\text { RSL } \\
\text { Indicator }\end{array}$ & $\begin{array}{l}\text { Elevation } \\
\text { (m) }\end{array}$ & $\begin{array}{l}\text { Paleo RSL } \\
\text { (m) }\end{array}$ & Dating (Original ID) & $\begin{array}{l}\text { Age (ka or } \\
\text { MIS) }\end{array}$ & $\begin{array}{l}\text { Quality } \\
\text { (RSL, } \\
\text { Age) }\end{array}$ & Reference(s) \\
\hline & $\begin{array}{l}44.548589 \\
11.940942\end{array}$ & $\begin{array}{l}\text { 222-S2 Voltana } \\
\text { Core S2 of the } \\
\text { geological map sheet } \\
222 \text { "Lugo di } \\
\text { Romagna", NE of } \\
\text { Voltana; ground }+5.0 \mathrm{~m} \\
\text { asl }\end{array}$ & & & & & $\begin{array}{l}\text { STRAT (Tyrrhenian } \\
\text { Adriatic (Eemian pollen } \\
\text { unit) }\end{array}$ & MIS 5e & & Amorosi et al., 2004 \\
\hline 1313 & $\begin{array}{l}44.622539 \\
11.813075\end{array}$ & $\begin{array}{l}\text { 204-S17 Argenta } \\
\text { Core S-17 of the } \\
\text { geological map sheet } \\
204 \text { "Portomaggiore" } \\
\text { near Argenta; ground } \\
\text { surface }+7.0 \mathrm{~m}\end{array}$ & SLI & BRK & $-54.5 \pm 5.3$ & $-54.5 \pm 11.3$ & $\begin{array}{l}\text { STRAT (Tyrrhenian } \\
\text { Adriatic (Eemian pollen } \\
\text { unit) }\end{array}$ & MIS 5e & 3,3 & $\begin{array}{l}\text { Campo et al., } 2020 \\
\text { Calabrese et al., } 2006\end{array}$ \\
\hline 1312 & $\begin{array}{l}44.664156 \\
11.785878\end{array}$ & $\begin{array}{l}\text { 204-S4 Consandolo } \\
\text { Core S4, geological map } \\
\text { sheet } 204 \\
\text { "Portomaggiore" near } \\
\text { Consandolo; ground } \\
\text { surface at }+1.7 \mathrm{~m} \mathrm{sl}\end{array}$ & SLI & LAG & $-50.8 \pm 1.8$ & $-49.8 \pm 2.1$ & $\begin{array}{l}\text { STRAT (Tyrrhenian } \\
\text { Adriatic (Eemian pollen } \\
\text { unit) }\end{array}$ & MIS 5e & 4,4 & $\begin{array}{l}\text { Campo et al., } 2020 \\
\text { Calabrese et al., } 2006\end{array}$ \\
\hline 1315 & $\begin{array}{l}44.720783 \\
11.680283\end{array}$ & $\begin{array}{l}\text { 204-S15 Marrara } \\
\text { Core S-15 of the } \\
\text { geological map, sheet } \\
204 \text { "Portomaggiore" } \\
\text { near Marrara; ground } \\
\text { surface }+5.3 \mathrm{~m}\end{array}$ & TLI & & $-48.1 \pm 1$ & $0 \pm 0$ & $\begin{array}{l}\text { STRAT (Tyrrhenian } \\
\text { Adriatic (Eemian pollen } \\
\text { unit) }\end{array}$ & MIS 5e & 0,3 & $\begin{array}{l}\text { Campo et al., } 2020 \\
\text { Calabrese et al., } 2006\end{array}$ \\
\hline 1314 & $\begin{array}{l}44.699889 \\
11.918761\end{array}$ & $\begin{array}{l}\text { Core 204-S16 } \\
\text { Core S-16 of the } \\
\text { geological map, sheet } \\
204 \text { "Portomaggiore" } \\
\text { near Ostellato; ground } \\
\text { surface }+7.0 \mathrm{~m}\end{array}$ & SLI & BRK & $-77 \pm 2.6$ & $-77 \pm 10.3$ & $\begin{array}{l}\text { STRAT (Tyrrhenian } \\
\text { Adriatic (Eemian pollen } \\
\text { unit) }\end{array}$ & MIS 5e & 3,3 & $\begin{array}{l}\text { Campo et al., } 2020 \\
\text { Calabrese et al., } 2006\end{array}$ \\
\hline & & 205-S10 Comacchio & & & & & ESR (K-4383) & $124 \pm 20$ & & \\
\hline 1309 & $\begin{array}{l}44.662453 \\
12.18375\end{array}$ & $\begin{array}{l}\text { Core } S 10 \text { of the } \\
\text { geological map sheet } \\
\text { of Comacchio", south } \\
-0.8 \mathrm{~m} \text { asl }\end{array}$ & SLI & BRK & $-112.5 \pm 1$ & $-112.5 \pm 10$ & $\begin{array}{l}\text { STRAT (Tyrrhenian } \\
\text { Adriatic (Eemian pollen } \\
\text { unit) }\end{array}$ & MIS 5e & 3,5 & $\begin{array}{l}\text { Ferranti et al., } 2006 \\
\text { Amorosi et al., } 2004 \\
\text { Campo et al., } 2020\end{array}$ \\
\hline 1307 & $\begin{array}{l}44.841086 \\
12.191919\end{array}$ & $\begin{array}{l}\text { Core F187_S1 } \\
\text { Valle Giralda, Foglio } \\
\text { CARG } 187 \text { Codigoro, } \\
\text { near Abazia of } \\
\text { Pomposa; ground at }-2.3 \\
\text { m asl. }\end{array}$ & MLI & & $-102 \pm 1$ & & $\begin{array}{l}\text { STRAT (Subsintema di } \\
\text { Villa Verucchio } \\
\text { (AES7) ) }\end{array}$ & MIS 6 & 0,2 & $\begin{array}{l}\text { Cibin and Stefani, } 2009 \\
\text { Campo et al., } 2020\end{array}$ \\
\hline
\end{tabular}




\begin{tabular}{|c|c|c|c|c|c|c|c|c|c|c|}
\hline $\begin{array}{l}\text { WALIS } \\
\text { ID }\end{array}$ & $\begin{array}{l}\text { Latitude } \\
\text { Longitude }\end{array}$ & Site & $\begin{array}{l}\text { Type of } \\
\text { datapoint }\end{array}$ & $\begin{array}{l}\text { RSL } \\
\text { Indicator }\end{array}$ & $\begin{array}{l}\text { Elevation } \\
\text { (m) }\end{array}$ & $\begin{array}{l}\text { Paleo RSL } \\
\text { (m) }\end{array}$ & Dating (Original ID) & $\begin{array}{l}\text { Age (ka or } \\
\text { MIS) }\end{array}$ & $\begin{array}{l}\text { Quality } \\
\text { (RSL, } \\
\text { Age) }\end{array}$ & Reference(s) \\
\hline 1306 & $\begin{array}{l}45.29235 \\
11.9007\end{array}$ & $\begin{array}{l}\text { Ca' Borille } \\
\text { Core Ca' Borille, near } \\
\text { the town of Bovolenta; } \\
\text { ground surface at }+4.2 \\
\text { m asl. }\end{array}$ & TLI & & $-44.5 \pm 1$ & & $\begin{array}{l}\text { STRAT (Tyrrhenian } \\
\text { Adriatic (Eemian pollen } \\
\text { unit) }\end{array}$ & MIS 5e & 0,3 & Cucato et al., 2012 \\
\hline 1304 & $\begin{array}{l}45.352278 \\
12.1375\end{array}$ & $\begin{array}{l}\text { Valle Averto } \\
\text { Core CARG } 11 \text { in Valle } \\
\text { Averto, at the boundary } \\
\text { of Venice Lagoon }\end{array}$ & SLI & LAG & $-61.3 \pm 1.3$ & $-60.3 \pm 1.6$ & $\begin{array}{l}\text { STRAT (Tyrrhenian } \\
\text { Adriatic (Eemian pollen } \\
\text { unit) }\end{array}$ & MIS 5e & 4,3 & $\begin{array}{l}\text { Tosi et al., 2007b } \\
\text { Antonioli et al., } 2009 \\
\text { Fontana et al., } 2010\end{array}$ \\
\hline 1305 & $\begin{array}{l}45.336544 \\
12.326236\end{array}$ & $\begin{array}{l}\text { Malamocco } \\
\text { Core at the mouth of } \\
\text { Malamocco Inlet in the } \\
\text { Venice Lagoon }\end{array}$ & MLI & & $-86 \pm 1$ & & $\begin{array}{l}\text { STRAT (Tyrrhenian } \\
\text { Adriatic (Eemian pollen } \\
\text { unit) }\end{array}$ & MIS 5e & 0,2 & $\begin{array}{l}\text { Antonioli et al., } 2009 \\
\text { Fontana et al., } 2010\end{array}$ \\
\hline 1303 & $\begin{array}{l}45.438569 \\
12.309925\end{array}$ & $\begin{array}{l}\text { VE-1 } \\
\text { Core Venezia1; Venice, } \\
\text { Island of Tronchetto }\end{array}$ & MLI & & $-60 \pm 1$ & $-60 \pm 0$ & $\begin{array}{l}\text { STRAT (Tyrrhenian } \\
\text { Adriatic (Eemian pollen } \\
\text { unit) }\end{array}$ & MIS 5e & 0,3 & $\begin{array}{l}\text { Massari et al., } 2004 \\
\text { Antonioli et al., } 2009\end{array}$ \\
\hline 1302 & $\begin{array}{l}45.555101 \\
12.456551\end{array}$ & $\begin{array}{l}\text { Portegrandi } \\
\text { Core CARG12 near the } \\
\text { village of Portegrandi, } \\
\text { near Venice Lagoon; } \\
\text { ground at } 0.0 \mathrm{~m} \text { asl. }\end{array}$ & MLI & & $-55 \pm 1$ & & $\begin{array}{l}\text { STRAT (Tyrrhenian } \\
\text { Adriatic (Eemian pollen } \\
\text { unit) }\end{array}$ & MIS 5e & 0,3 & $\begin{array}{l}\text { Tosi et al., 2007a } \\
\text { Antonioli et al., } 2009 \\
\text { Fontana et al., } 2010\end{array}$ \\
\hline 1295 & $\begin{array}{l}45.621667 \\
12.756111\end{array}$ & $\begin{array}{l}\text { TdM Torre di Mosto } \\
\text { Core TdM, drilled in } \\
\text { Senzielli of Torre di } \\
\text { Mosto; ground surface } \\
\text { at }-1.4 \mathrm{~m} \text { asl. }\end{array}$ & MLI & & - & & $\begin{array}{l}\text { STRAT (Tyrrhenian } \\
\text { Adriatic (Eemian pollen } \\
\text { unit) }\end{array}$ & MIS 5e & 0,4 & $\begin{array}{l}\text { Fontana et al., } 2012 \\
\text { Antonioli et al., } 2009 \\
\text { Fontana et al., } 2010\end{array}$ \\
\hline 793 & $\begin{array}{l}45.774444 \\
12.713333\end{array}$ & $\begin{array}{l}\text { PRA Belfiore } \\
\text { Core PRA, drilled in the } \\
\text { village of Belfiore near } \\
\text { Pramaggiore; ground } \\
\text { surface at }+4.0 \mathrm{~m} \text { asl. }\end{array}$ & SLI & LAG & $-54.6 \pm 10$ & $-54.1 \pm 10$ & $\begin{array}{l}\text { STRAT (Tyrrhenian } \\
\text { Adriatic (Eemian pollen } \\
\text { unit) }\end{array}$ & MIS 5e & 4,4 & $\begin{array}{l}\text { Fontana et al., } 2012 \\
\text { Fontana et al., } 2010\end{array}$ \\
\hline 1289 & $\begin{array}{l}45.795333 \\
12.840977\end{array}$ & $\begin{array}{l}\text { CNC4 Concordia } \\
\text { Sagittaria } \\
\text { Core drilled near the } \\
\text { theatre of Roman age. } \\
\text { Ground surface at }+2.0 \\
\mathrm{~m} \text { asl. }\end{array}$ & SLI & LAG & $-37 \pm 1$ & $-36.5 \pm 1.1$ & $\begin{array}{l}\text { STRAT (Tyrrhenian } \\
\text { Adriatic (Eemian pollen } \\
\text { unit) }\end{array}$ & MIS 5e & 4,4 & $\begin{array}{l}\text { Fontana et al., } 2012 \\
\text { Antonioli et al., } 2009 \\
\text { Fontana et al., } 2010\end{array}$ \\
\hline 1294 & $\begin{array}{l}45.713992 \\
12.94864\end{array}$ & $\begin{array}{l}\text { LUG Lugugnana } \\
\text { Core LUG, drilled near } \\
\text { the village of Marina di } \\
\text { Lugugnana; ground at } \\
+1.0 \text { m asl. }\end{array}$ & MLI & & $-41 \pm 4.6$ & & $\begin{array}{l}\text { STRAT (Tyrrhenian } \\
\text { Adriatic (Eemian pollen } \\
\text { unit) }\end{array}$ & MIS 5e & 0,3 & $\begin{array}{l}\text { Fontana et al., } 2012 \\
\text { Fontana et al., } 2010\end{array}$ \\
\hline 1293 & $\begin{array}{l}45.63955 \\
12.954483\end{array}$ & $\begin{array}{l}\text { VV Valle Vecchia } \\
\text { Core VV in the farm } \\
\text { Valle Vecchia, claimed }\end{array}$ & MLI & & $-47 \pm 2.6$ & & $\begin{array}{l}\text { STRAT (Tyrrhenian } \\
\text { Adriatic (Eemian pollen } \\
\text { unit) }\end{array}$ & MIS 5e & 0,3 & $\begin{array}{l}\text { Fontana et al., } 2012 \\
\text { Fontana et al., } 2010\end{array}$ \\
\hline
\end{tabular}




\begin{tabular}{|c|c|c|c|c|c|c|c|c|c|c|}
\hline $\begin{array}{l}\text { WALIS } \\
\text { ID }\end{array}$ & $\begin{array}{l}\text { Latitude } \\
\text { Longitude }\end{array}$ & Site & $\begin{array}{l}\text { Type of } \\
\text { datapoint }\end{array}$ & $\begin{array}{l}\text { RSL } \\
\text { Indicator }\end{array}$ & $\begin{array}{l}\text { Elevation } \\
(\mathbf{m})\end{array}$ & $\begin{array}{l}\text { Paleo RSL } \\
\text { (m) }\end{array}$ & Dating (Original ID) & $\begin{array}{l}\text { Age (ka or } \\
\text { MIS) }\end{array}$ & $\begin{array}{l}\text { Quality } \\
\text { (RSL, } \\
\text { Age) }\end{array}$ & Reference(s) \\
\hline & & $\begin{array}{l}\text { in 20th century. Ground } \\
0.0 \mathrm{~m} \text { asl }\end{array}$ & & & & & & & & \\
\hline \multicolumn{11}{|c|}{ Italy (Friuli-Venezia-Giulia) } \\
\hline 1301 & $\begin{array}{l}45.836747 \\
12.670481\end{array}$ & $\begin{array}{l}\text { AZX Azzano } \\
\text { Core AZX, Casa De } \\
\text { Vito near Panigai of } \\
\text { Azzano Decimo; ground } \\
+9,8 \mathrm{~m} \text { asl. }\end{array}$ & TLI & & $-43.9 \pm 1.7$ & & $\begin{array}{l}\text { STRAT (Tyrrhenian } \\
\text { Adriatic (Eemian pollen } \\
\text { unit) }\end{array}$ & MIS 5e & 3,5 & $\begin{array}{l}\text { Pini et al. } 2009 \\
\text { Fontana et al., } 2010 \\
\text { Zanferrari et al. } 2008\end{array}$ \\
\hline 1291 & $\begin{array}{l}45.78375 \\
13.08425\end{array}$ & $\begin{array}{l}\text { Piancada } \\
\text { Piancada town. Probably } \\
\text { elevation of the surface } \\
+2 \mathrm{~m} \text { asl. }\end{array}$ & SLI & LAG & $-48 \pm 9.2$ & $-47 \pm 9.3$ & $\begin{array}{l}\text { STRAT (Tyrrhenian } \\
\text { Adriatic (Eemian pollen } \\
\text { unit) }\end{array}$ & MIS 5e & 3,3 & $\begin{array}{l}\text { Feruglio } 1936 \\
\text { Antonioli et al., } 2009 \\
\text { Fontana et al., } 2010 \\
\text { Lipparini } 1936\end{array}$ \\
\hline 1292 & $\begin{array}{l}45.765014 \\
13.102561\end{array}$ & $\begin{array}{l}\text { PNC1 Piancada } \\
\text { Core PNC1 in Marianis } \\
\text { of Piancada, } \\
\text { municipality of } \\
\text { Palazzolo dello Stella; } \\
\text { ground at }+1.4 \mathrm{~m} \text { asl }\end{array}$ & SLI & LAG & $-42.1 \pm 6.8$ & $-41.1 \pm 6.9$ & $\begin{array}{l}\text { STRAT (Tyrrhenian } \\
\text { Adriatic (Eemian pollen } \\
\text { unit) }\end{array}$ & MIS 5e & 4,3 & $\begin{array}{l}\text { Antonioli et al., } 2009 \\
\text { Fontana et al., } 2010\end{array}$ \\
\hline 1290 & $\begin{array}{l}45.74849 \\
13.366341\end{array}$ & $\begin{array}{l}\text { BLG1, Beligna } \\
\text { Core BLG1 (Beligna), } \\
\text { in the site of Beligna of } \\
\text { Aquileia. Ground } \\
\text { surface at }+2.0 \mathrm{~m} \text { asl. }\end{array}$ & SLI & LAG & $-44 \pm 8.1$ & $-43 \pm 8.1$ & $\begin{array}{l}\text { STRAT (Tyrrhenian } \\
\text { Adriatic (Eemian pollen } \\
\text { unit) }\end{array}$ & MIS 5e & 3,3 & Fontana et al., 2010 \\
\hline \multicolumn{11}{|c|}{ France (Corse) } \\
\hline 277 & $\begin{array}{l}41.3711 \\
9.2223\end{array}$ & Piantarella & MLI & & $3.3 \pm 0.2$ & & $\begin{array}{l}\text { STRAT (P. latus or } \\
\text { Senegalese fauna) }\end{array}$ & MIS 5 & 0,3 & Nesteroff, 1984 \\
\hline 321 & $\begin{array}{l}41.47 \\
9.075\end{array}$ & Figari & SLI & LAG & $3 \pm 0.6$ & $4 \pm 1.2$ & $\begin{array}{l}\text { OTHER (Limiting } \\
\text { radiocarbon date) }\end{array}$ & $>$ MIS 1 & 4,2 & $\begin{array}{l}\text { Conchon, } 1999 \\
\text { Delibrias et al., } 1972 \text { Radiocarbon } \\
\text { Nesteroff, } 1984\end{array}$ \\
\hline 323 & $\begin{array}{l}41.47 \\
9.01\end{array}$ & Arbitru & MLI & & $2 \pm 0.4$ & & $\begin{array}{l}\text { OTHER (Limiting } \\
\text { radiocarbon date) }\end{array}$ & $>$ MIS 1 & 0,2 & $\begin{array}{l}\text { Conchon, } 1999 \\
\text { Delibrias et al., } 1972 \text { Radiocarbon } \\
\text { Nesteroff, } 1984\end{array}$ \\
\hline 303 & $\begin{array}{l}41.76 \\
8.71\end{array}$ & $\begin{array}{l}\text { Ajaccio Gulf } \\
\text { Capo Moru }\end{array}$ & MLI & & $2.5 \pm 0.5$ & & $\begin{array}{l}\text { OTHER (Limiting } \\
\text { radiocarbon date) }\end{array}$ & $>$ MIS 1 & 0,2 & $\begin{array}{l}\text { Conchon, } 1999 \\
\text { Nesteroff, } 1984\end{array}$ \\
\hline 299 & $\begin{array}{l}42.6716 \\
9.2997\end{array}$ & St Florent & MLI & & $1.5 \pm 0.3$ & & AAR (St. Florent, ) & MIS 5e & 0,3 & $\begin{array}{l}\text { Conchon, 1999 } \\
\text { Hearty, 1987a } \\
\text { Ottman, 1954 }\end{array}$ \\
\hline 931 & $\begin{array}{l}42.96 \\
9.45 \\
\end{array}$ & $\begin{array}{l}\text { Macinaggio } \\
\text { Tamarone }\end{array}$ & SLI & LIT & $6 \pm 0.5$ & $6.3 \pm 0.6$ & $\begin{array}{l}\text { STRAT ( } P \text {. latus } \text { or } \\
\text { Senegalese fauna) }\end{array}$ & MIS 5 & 5,3 & Ottman, 1954 \\
\hline 322 & $\begin{array}{l}42.06 \\
9.46\end{array}$ & Urbino & MLI & & $1 \pm 0.2$ & & $\begin{array}{l}\text { OTHER (Limiting } \\
\text { radiocarbon date) }\end{array}$ & $>$ MIS 1 & 0,2 & $\begin{array}{l}\text { Conchon, } 1999 \\
\text { Delibrias et al., } 1972 \text { Radiocarbon } \\
\text { Nesteroff, } 1984\end{array}$ \\
\hline \multicolumn{11}{|c|}{ Italy (Sardinia) } \\
\hline 432 & $\begin{array}{l}40.02 \\
9.7\end{array}$ & Pedralonga & SLI & NTC & $7.4 \pm 0.2$ & $7.4 \pm 0.4$ & $\begin{array}{l}\text { STRAT (P. latus or } \\
\text { Senegalese fauna) }\end{array}$ & MIS 5 & 5,2 & Antonioli et al., 2018 \\
\hline
\end{tabular}




\begin{tabular}{|c|c|c|c|c|c|c|c|c|c|c|}
\hline $\begin{array}{l}\text { WALIS } \\
\text { ID }\end{array}$ & $\begin{array}{l}\text { Latitude } \\
\text { Longitude }\end{array}$ & Site & $\begin{array}{l}\text { Type of } \\
\text { datapoint }\end{array}$ & $\begin{array}{l}\text { RSL } \\
\text { Indicator }\end{array}$ & $\begin{array}{l}\text { Elevation } \\
(\mathbf{m})\end{array}$ & $\begin{array}{l}\text { Paleo RSL } \\
\text { (m) }\end{array}$ & Dating (Original ID) & $\begin{array}{l}\text { Age (ka or } \\
\text { MIS) }\end{array}$ & $\begin{array}{l}\text { Quality } \\
\text { (RSL, } \\
\text { Age) }\end{array}$ & Reference(s) \\
\hline 435 & $\begin{array}{l}40.09 \\
9.72\end{array}$ & Capo Monte Santu & SLI & NTC & $7.9 \pm 0.1$ & $7.9 \pm 0.3$ & $\begin{array}{l}\text { STRAT (P. latus or } \\
\text { Senegalese fauna) }\end{array}$ & MIS 5 & 5,2 & Antonioli et al., 2018 \\
\hline 434 & $\begin{array}{l}40.24 \\
9.62\end{array}$ & Orosei Nord & SLI & NTC & $9.5 \pm 0.2$ & $9.5 \pm 0.4$ & $\begin{array}{l}\text { STRAT (P. latus or } \\
\text { Senegalese fauna) }\end{array}$ & MIS 5 & 5,2 & Antonioli et al., 2018 \\
\hline 292 & $\begin{array}{l}40.8878 \\
9.6928 \\
\end{array}$ & $\begin{array}{l}\text { Tavolara } \\
\text { Punta la mandria }\end{array}$ & MLI & & $1.8 \pm 0.3$ & & AAR (TV1) & $120 \pm 36$ & 0,3 & $\begin{array}{l}\text { Belluomini et al., } 1986 \\
\text { Belluomini and Delitala, } 1988\end{array}$ \\
\hline 431 & $\begin{array}{l}40.91 \\
9.74 \\
\end{array}$ & Tavolara & SLI & NTC & $6.7 \pm 0.4$ & $6.7 \pm 0.5$ & $\begin{array}{l}\text { STRAT ( } P . \text { latus or } \\
\text { Senegalese fauna) }\end{array}$ & MIS 5 & 5,2 & Antonioli et al., 2018 \\
\hline 430 & $\begin{array}{l}40.99 \\
9.66\end{array}$ & Capo Figari & SLI & NTC & $4.7 \pm 0.2$ & $4.7 \pm 0.4$ & $\begin{array}{l}\text { STRAT (P. latus or } \\
\text { Senegalese fauna) }\end{array}$ & MIS 5 & 5,2 & Antonioli et al., 2018 \\
\hline 289 & $\begin{array}{l}41.2368 \\
9.1656\end{array}$ & $\begin{array}{l}\text { Capo Testa_santa } \\
\text { Reparata } \\
\text { TLI } \\
\end{array}$ & TLI & & $0.3 \pm 0.5$ & & AAR (Sequence 3) & $90 \pm 27$ & 0,3 & Kindler et al. 1997 \\
\hline 290 & $\begin{array}{l}41.2365 \\
9.1649\end{array}$ & $\begin{array}{l}\text { Capo Testa_santa } \\
\text { Reparata } \\
\text { MLI } \\
\end{array}$ & MLI & & $3 \pm 0.5$ & & AAR (Sequence 3) & $90 \pm 27$ & 0,3 & Kindler et al. 1997 \\
\hline \multirow{2}{*}{300} & \multirow{2}{*}{$\begin{array}{l}40.9295 \\
8.8052\end{array}$} & \multirow{2}{*}{$\begin{array}{l}\text { Badesi Mare } \\
\text { San Pietro a Mare }\end{array}$} & \multirow{2}{*}{ MLI } & & \multirow{2}{*}{$1.5 \pm 0.3$} & & AAR (SPM1) & MIS 5e & \multirow{2}{*}{0,3} & \multirow{2}{*}{$\begin{array}{l}\text { Belluomini and Delitala, } 1988 \\
\text { Belluomini et al., } 1986\end{array}$} \\
\hline & & & & & & & AAR (SPM2) & MIS 5e & & \\
\hline 388 & $\begin{array}{l}40.63 \\
8.18 \\
\end{array}$ & Cala Viola & SLI & BRK & $3.5 \pm 0.2$ & $3.4 \pm 0.3$ & LUM (CVL3) & $136 \pm 8$ & 5,3 & Casini et al., 2020 \\
\hline 382 & $\begin{array}{l}40.6 \\
8.14 \\
\end{array}$ & Capo Caccia 1 & SLI & NTC & $3.8 \pm 0.1$ & $3.8 \pm 0.3$ & $\begin{array}{l}\text { STRAT ( } P . \text { latus } \text { or } \\
\text { Senegalese fauna) }\end{array}$ & MIS 5 & 5,2 & Antonioli et al., 2018 \\
\hline 383 & $\begin{array}{l}40.56 \\
8.16 \\
\end{array}$ & Capo Caccia 2 & SLI & NTC & $3.5 \pm 0.1$ & $3.5 \pm 0.3$ & $\begin{array}{l}\text { STRAT ( } P . \text { latus or } \\
\text { Senegalese fauna) }\end{array}$ & MIS 5 & 5,2 & Antonioli et al., 2018 \\
\hline 234 & $\begin{array}{l}40.5503 \\
8.3203 \\
\end{array}$ & El trò & SLI & ARI & $3.5 \pm 0.1$ & $3.3 \pm 0.3$ & LUM (CV2_feldspar) & $127 \pm 14$ & 5,4 & Sechi et al., 2020 \\
\hline 235 & $\begin{array}{l}40.5179 \\
8.3328 \\
\end{array}$ & Punta Padre Bellu & SLI & ARI & $3.3 \pm 0.1$ & $3.1 \pm 0.3$ & LUM (PPB1_feldspar) & $135 \pm 8$ & 5,4 & Sechi et al., 2020 \\
\hline 236 & $\begin{array}{l}40.5089 \\
8.3391 \\
\end{array}$ & Baurantinu & SLI & ARI & $3.5 \pm 0.1$ & $3.3 \pm 0.3$ & LUM (BUR_feldspar) & $119 \pm 6$ & 5,5 & Sechi et al., 2020 \\
\hline \multirow{2}{*}{241} & \multirow{2}{*}{$\begin{array}{l}40.3121 \\
8.4636\end{array}$} & \multirow{2}{*}{$\begin{array}{l}\text { Bosa } \\
\text { Sect } 1\end{array}$} & \multirow{2}{*}{ MLI } & & \multirow{2}{*}{$1.5 \pm 0.1$} & & LUM (SD2_quartz) & $93 \pm 7$ & \multirow{2}{*}{0,5} & \multirow{2}{*}{ Sechi et al., 2020} \\
\hline & & & & & & & LUM (SD2_feldspar) & $99 \pm 6$ & & \\
\hline 243 & $\begin{array}{l}40.3121 \\
8.4636 \\
\end{array}$ & $\begin{array}{l}\text { Bosa } \\
\text { Sect } 2 \\
\end{array}$ & MLI & & $2 \pm 0.1$ & & LUM (PA2_feldspar) & $139 \pm 13$ & 0,4 & Sechi et al., 2020 \\
\hline 244 & $\begin{array}{l}40.2428 \\
8.4722 \\
\end{array}$ & $\begin{array}{l}\text { Bosa } \\
\text { Sect } 3\end{array}$ & SLI & ARI & $3 \pm 0.1$ & $2.8 \pm 0.3$ & LUM (SD1) & $125 \pm 8$ & 5,4 & Sechi et al., 2020 \\
\hline 238 & $\begin{array}{l}40.2428 \\
8.4722 \\
\end{array}$ & Porto Alabe & SLI & ARI & $3.3 \pm 0.1$ & $3.1 \pm 0.3$ & LUM (PA3_feldspar) & $134 \pm 13$ & 5,4 & Sechi et al., 2020 \\
\hline 262 & $\begin{array}{l}39.8859 \\
8.4333 \\
\end{array}$ & $\begin{array}{l}\text { San Giovanni del Sinis } \\
1\end{array}$ & SLI & BRK & $1.8 \pm 0.3$ & $2.8 \pm 2$ & U-series (CLD1) & $151 \pm 7.8$ & 3,2 & Carboni et al., 2014 \\
\hline 271 & $\begin{array}{l}39.8861 \\
8.4332 \\
\end{array}$ & $\begin{array}{l}\text { San Giovanni del Sinis } \\
4\end{array}$ & SLI & BRK & $5.2 \pm 0.2$ & $5.7 \pm 1.5$ & LUM (SGS2) & $120 \pm 10$ & 5,5 & Andreucci et al., 2009 \\
\hline 272 & $\begin{array}{l}39.8829 \\
8.4357 \\
\end{array}$ & $\begin{array}{l}\text { San Giovanni del Sinis } \\
5\end{array}$ & MLI & & $2.5 \pm 0.2$ & & LUM (SGS3) & $100 \pm 5$ & 0,5 & Andreucci et al., 2009 \\
\hline
\end{tabular}




\begin{tabular}{|c|c|c|c|c|c|c|c|c|c|c|}
\hline $\begin{array}{l}\text { WALIS } \\
\text { ID }\end{array}$ & $\begin{array}{l}\text { Latitude } \\
\text { Longitude }\end{array}$ & Site & $\begin{array}{l}\text { Type of } \\
\text { datapoint }\end{array}$ & $\begin{array}{l}\text { RSL } \\
\text { Indicator }\end{array}$ & $\begin{array}{l}\text { Elevation } \\
(\mathbf{m})\end{array}$ & $\begin{array}{l}\text { Paleo RSL } \\
\text { (m) }\end{array}$ & Dating (Original ID) & $\begin{array}{l}\text { Age (ka or } \\
\text { MIS) }\end{array}$ & $\begin{array}{l}\text { Quality } \\
\text { (RSL, } \\
\text { Age) }\end{array}$ & Reference(s) \\
\hline 265 & $\begin{array}{l}39.8807 \\
8.4364 \\
\end{array}$ & $\begin{array}{l}\text { San Giovanni del Sinis } \\
3\end{array}$ & SLI & BRK & $0.8 \pm 0.3$ & $1.8 \pm 2$ & U-series (CLD3) & $139 \pm 4.1$ & 4,2 & Carboni et al., 2014 \\
\hline 266 & $\begin{array}{l}39.858 \\
8.4355 \\
\end{array}$ & Faro Capo San Marco & SLI & BRK & $3 \pm 0.5$ & $4 \pm 2.1$ & U-series (CLD5) & $135 \pm 4.1$ & 3,2 & Carboni et al., 2014 \\
\hline 261 & $\begin{array}{l}39.8637 \\
8.4391 \\
\end{array}$ & Capo San Marco & MLI & & $2.5 \pm 0.3$ & & $\begin{array}{l}\text { U-series (Capo San } \\
\text { Marco) }\end{array}$ & $70 \pm 4$ & 0,4 & D'Orefice et al., 2012 \\
\hline 263 & $\begin{array}{l}39.8688 \\
8.4398 \\
\end{array}$ & $\begin{array}{l}\text { San Giovanni del Sinis } \\
2\end{array}$ & SLI & BRK & $1 \pm 0.3$ & $2 \pm 2$ & U-series (CLD2) & $128 \pm 4.1$ & 4,3 & Carboni et al., 2014 \\
\hline 437 & $\begin{array}{l}39.39 \\
8.38 \\
\end{array}$ & Buggerru & SLI & NTC & $3.5 \pm 0.1$ & $3.5 \pm 0.3$ & $\begin{array}{l}\text { STRAT (P. latus or } \\
\text { Senegalese fauna) }\end{array}$ & MIS 5 & 5,2 & Antonioli et al., 2018 \\
\hline 436 & $\begin{array}{l}39.33 \\
8.4 \\
\end{array}$ & Masua & SLI & NTC & $3.2 \pm 0.1$ & $3.2 \pm 0.3$ & $\begin{array}{l}\text { STRAT ( } P . \text { latus or } \\
\text { Senegalese fauna) }\end{array}$ & MIS 5 & 5,2 & Antonioli et al., 2018 \\
\hline 296 & $\begin{array}{l}39.0063 \\
8.3847 \\
\end{array}$ & $\begin{array}{l}\text { Sant'Antioco } \\
\text { Cala Sapone }\end{array}$ & SLI & BRK & $7.5 \pm 0.3$ & $8 \pm 2.5$ & $\begin{array}{l}\text { STRAT ( } P . \text { latus } \text { or } \\
\text { Senegalese fauna) }\end{array}$ & MIS 5 & 3,3 & Orrù et al., 2011 \\
\hline \multirow[b]{2}{*}{433} & \multirow{2}{*}{$\begin{array}{l}38.98 \\
8.44\end{array}$} & \multirow[b]{2}{*}{ Sant'Antioco } & \multirow[b]{2}{*}{ SLI } & \multirow[b]{2}{*}{ NTC } & \multirow[b]{2}{*}{$2.8 \pm 0.2$} & \multirow[b]{2}{*}{$2.8 \pm 0.5$} & AAR (S10) & MIS 5e & \multirow[b]{2}{*}{4,2} & \multirow{2}{*}{$\begin{array}{l}\text { Antonioli et al., } 2018 \\
\text { Ulzega and Hearty, } 1986\end{array}$} \\
\hline & & & & & & & $\begin{array}{l}\text { STRAT ( } P . \text { latus or } \\
\text { Senegalese fauna) }\end{array}$ & MIS 5 & & \\
\hline 295 & $\begin{array}{l}38.8918 \\
8.8007 \\
\end{array}$ & Capo Malfatano & SLI & BRK & $2 \pm 0.3$ & $2.5 \pm 2.5$ & $\begin{array}{l}\text { STRAT ( } P \text {. latus or } \\
\text { Senegalese fauna) }\end{array}$ & MIS 5 & 3,3 & Orrù et al., 2014 \\
\hline 1336 & $\begin{array}{l}38.985 \\
8.99 \\
\end{array}$ & Nora & MLI & & $1.5 \pm 0.5$ & & AAR (S7) & $125 \pm 37.5$ & 0,3 & $\begin{array}{l}\text { Kindler et al. } 1997 \\
\text { Ulzega and Hearty, } 1986\end{array}$ \\
\hline 1337 & $\begin{array}{l}38.986 \\
9\end{array}$ & $\begin{array}{l}\text { Nora } \\
\text { Is Fradis }\end{array}$ & SLI & BRK & $1.8 \pm 0.5$ & $3.3 \pm 0.7$ & AAR (S7) & $125 \pm 37.5$ & 4,3 & $\begin{array}{l}\text { Kindler et al. } 1997 \\
\text { Ulzega and Hearty, } 1986\end{array}$ \\
\hline 279 & $\begin{array}{l}38.9867 \\
9.006 \\
\end{array}$ & Nora-Is Fradis & MLI & & $1 \pm 0.2$ & & $\begin{array}{l}\text { STRAT ( } P . \text { latus or } \\
\text { Senegalese fauna) }\end{array}$ & MIS 5 & 0,3 & Ulzega and Hearty, 1986 \\
\hline 278 & $\begin{array}{l}39.2182 \\
9.0442 \\
\end{array}$ & Sa Illetta & MLI & & $1.5 \pm 0.2$ & & U-series (S6) & $149 \pm 10$ & 0,2 & Ulzega and Hearty, 1986 \\
\hline 1338 & $\begin{array}{l}39.187 \\
9.141\end{array}$ & $\begin{array}{l}\text { Cagliari } \\
\text { Marina Piccola }\end{array}$ & SLI & BRK & $2.5 \pm 0.5$ & $3 \pm 1$ & AAR (MP2) & $125 \pm 37.5$ & 4,3 & $\begin{array}{l}\text { Stocchi et al, } 2018 \\
\text { Hearty et al., 1986a } \\
\text { Ulzega and Hearty, } 1986\end{array}$ \\
\hline 132 & $\begin{array}{l}39.184 \\
9.149\end{array}$ & $\begin{array}{l}\text { Cagliari } \\
\text { Cala Mosca }\end{array}$ & SLI & BRK & $2.8 \pm 0.5$ & $3.3 \pm 1$ & U-series (S-C-4) & $138 \pm 7$ & 4,4 & $\begin{array}{l}\text { Stocchi et al, } 2018 \\
\text { Hearty et al., } 1986 a \\
\text { Ulzega and Hearty, } 1986 \\
\end{array}$ \\
\hline \multirow{4}{*}{276} & \multirow{4}{*}{$\begin{array}{l}39.2217 \\
9.1663\end{array}$} & \multirow{4}{*}{ Is Arenas } & \multirow{4}{*}{ SLI } & \multirow{4}{*}{ BRK } & \multirow{4}{*}{$3 \pm 0.2$} & \multirow{4}{*}{$3.5 \pm 1.5$} & ESR (K-5741) & $103 \pm 8.6$ & \multirow{4}{*}{4,3} & \multirow{4}{*}{$\begin{array}{l}\text { Orru et al., } 2011 \\
\text { Orrù et al., } 2009\end{array}$} \\
\hline & & & & & & & ESR (K-5742) & $154.3 \pm 9.2$ & & \\
\hline & & & & & & & ESR (K-5744) & $81.2 \pm 8.1$ & & \\
\hline & & & & & & & ESR (K-5745) & $112.6 \pm 7.5$ & & \\
\hline 293 & $\begin{array}{l}39.1215 \\
9.5096 \\
\end{array}$ & $\begin{array}{l}\text { Capo Carbonara } \\
\text { Villasimius }\end{array}$ & SLI & BRK & $5.1 \pm 0.2$ & $5.6 \pm 2.5$ & $\begin{array}{l}\text { STRAT ( } P \text {. latus or } \\
\text { Senegalese fauna) }\end{array}$ & MIS 5 & 3,3 & Orrù et al., 1994 \\
\hline 294 & $\begin{array}{l}39.0866 \\
9.5345\end{array}$ & $\begin{array}{l}\text { Capo Carbonara } \\
\text { Isola dei cavoli-Cala } \\
\text { Ponente }\end{array}$ & SLI & BRK & $2.7 \pm 0.2$ & $3.2 \pm 2.5$ & $\begin{array}{l}\text { STRAT (P. latus or } \\
\text { Senegalese fauna) }\end{array}$ & MIS 5 & 3,3 & Orrù et al., 1994 \\
\hline
\end{tabular}




\begin{tabular}{|c|c|c|c|c|c|c|c|c|c|c|}
\hline $\begin{array}{l}\text { WALIS } \\
\text { ID }\end{array}$ & $\begin{array}{l}\text { Latitude } \\
\text { Longitude }\end{array}$ & Site & $\begin{array}{l}\text { Type of } \\
\text { datapoint }\end{array}$ & $\begin{array}{l}\text { RSL } \\
\text { Indicator }\end{array}$ & $\begin{array}{l}\text { Elevation } \\
\text { (m) }\end{array}$ & $\begin{array}{l}\text { Paleo RSL } \\
\text { (m) }\end{array}$ & Dating (Original ID) & $\begin{array}{l}\text { Age (ka or } \\
\text { MIS) }\end{array}$ & $\begin{array}{l}\text { Quality } \\
\text { (RSL, } \\
\text { Age) }\end{array}$ & Reference(s) \\
\hline 899 & $\begin{array}{l}37.72579 \\
12.4778 \\
\end{array}$ & $\begin{array}{l}\text { Marsala-Mazzaro del } \\
\text { Vallo } \\
\text { Torre Scibiliana }\end{array}$ & SLI & MT & $34 \pm 6.8$ & $34 \pm 6.9$ & $\begin{array}{l}\text { STRAT ( } P . \text { latus or } \\
\text { Senegalese fauna) }\end{array}$ & MIS 5 & 2,3 & $\begin{array}{l}\text { Ruggieri et al., } 1976 \\
\text { D'Angelo \& Vernuccio, } 1996 \\
\text { Ruggieri \& Unti, } 1988\end{array}$ \\
\hline 919 & $\begin{array}{l}37.890707 \\
12.473816 \\
\end{array}$ & Trapani Birgi & SLI & BRK & $2 \pm 0.4$ & $2.3 \pm 0.9$ & $\begin{array}{l}\text { STRAT ( } P . \text { latus or } \\
\text { Senegalese fauna) }\end{array}$ & MIS 5 & 4,3 & Ruggieri and Unti. 1974 \\
\hline 855 & $\begin{array}{l}37.930049 \\
12.350824\end{array}$ & $\begin{array}{l}\text { Favignana } \\
\text { Egadi archipelago }\end{array}$ & SLI & NTC & $12.5 \pm 0.5$ & $12.5 \pm 0.5$ & $\begin{array}{l}\text { STRAT (P. latus or } \\
\text { Senegalese fauna) }\end{array}$ & MIS 5 & 5,2 & $\begin{array}{l}\text { Malatesta et al., } 1957 \\
\text { Abate et al., } 1992\end{array}$ \\
\hline 841 & $\begin{array}{l}37.946651 \\
12.087998 \\
\end{array}$ & $\begin{array}{l}\text { Facciazzo } \\
\text { Marettimo Island }\end{array}$ & SLI & MT & $6 \pm 1.5$ & $6 \pm 2$ & LUM (Cala Rossa) & $111 \pm 20$ & 4,3 & $\begin{array}{l}\text { Antonioli et al., } 2002 \\
\text { Mauz et al., } 1997\end{array}$ \\
\hline 836 & $\begin{array}{l}37.950461 \\
12.082601\end{array}$ & $\begin{array}{l}\text { Cala Marino } \\
\text { Marettimo Island }\end{array}$ & SLI & NTC & $7.1 \pm 0.5$ & $7.1 \pm 0.5$ & LUM (Cala Rossa) & $111 \pm 20$ & 5,2 & $\begin{array}{l}\text { Antonioli et al., } 2002 \\
\text { Mauz et al., } 1997\end{array}$ \\
\hline 840 & $\begin{array}{l}37.952251 \\
12.081834 \\
\end{array}$ & $\begin{array}{l}\text { Cala Marino (II) } \\
\text { Marettimo Island }\end{array}$ & SLI & MT & $5 \pm 1.5$ & $5 \pm 2$ & LUM (Cala Rossa) & $111 \pm 20$ & 4,3 & $\begin{array}{l}\text { Antonioli et al., } 2002 \\
\text { Mauz et al., } 1997\end{array}$ \\
\hline 842 & $\begin{array}{l}37.950223 \\
12.077871 \\
\end{array}$ & $\begin{array}{l}\text { Cala Conca } \\
\text { Marettimo Island }\end{array}$ & SLI & MT & $4 \pm 1.5$ & $4 \pm 2$ & LUM (Cala Rossa) & $111 \pm 20$ & 4,3 & $\begin{array}{l}\text { Antonioli et al., } 2002 \\
\text { Mauz et al., } 1997\end{array}$ \\
\hline 843 & $\begin{array}{l}37.958734 \\
12.044408\end{array}$ & $\begin{array}{l}\text { Cala Nera } \\
\text { Marettimo Island }\end{array}$ & SLI & MT & $11 \pm 1.5$ & $11 \pm 2$ & LUM (Cala Rossa) & $111 \pm 20$ & 4,3 & $\begin{array}{l}\text { Antonioli et al., } 2002 \\
\text { Mauz et al., } 1997\end{array}$ \\
\hline 837 & $\begin{array}{l}37.972247 \\
12.040814 \\
\end{array}$ & $\begin{array}{l}\text { P. Pegna } \\
\text { Marettimo Island }\end{array}$ & SLI & NTC & $7.6 \pm 0.5$ & $7.6 \pm 0.5$ & LUM (Cala Rossa) & $111 \pm 20$ & 5,2 & $\begin{array}{l}\text { Antonioli et al., } 2002 \\
\text { Mauz et al., } 1997\end{array}$ \\
\hline 833 & $\begin{array}{l}37.992445 \\
12.041292 \\
\end{array}$ & $\begin{array}{l}\text { P.ta Due Frati } \\
\text { Marettimo Island }\end{array}$ & SLI & NTC & $8.2 \pm 0.5$ & $8.2 \pm 0.5$ & LUM (Cala Rossa) & $111 \pm 20$ & 5,2 & $\begin{array}{l}\text { Antonioli et al., } 2002 \\
\text { Malatesta et al., } 1957 \\
\text { Mauz et al., 1997 }\end{array}$ \\
\hline 834 & $\begin{array}{l}37.990279 \\
12.059884 \\
\end{array}$ & $\begin{array}{l}\text { Scalo Maestro } \\
\text { Marettimo Island }\end{array}$ & SLI & NTC & $7.5 \pm 0.5$ & $7.5 \pm 0.5$ & LUM (Cala Rossa) & $111 \pm 20$ & 5,2 & $\begin{array}{l}\text { Antonioli et al., } 2002 \\
\text { Mauz et al., } 1997\end{array}$ \\
\hline 838 & $\begin{array}{l}37.991736 \\
12.061893 \\
\end{array}$ & $\begin{array}{l}\text { Tuono } \\
\text { Marettimo Island }\end{array}$ & SLI & MT & $10.4 \pm 1.5$ & $10.4 \pm 2$ & LUM (Cala Rossa) & $111 \pm 20$ & 4,3 & $\begin{array}{l}\text { Antonioli et al., } 2002 \\
\text { Mauz et al., } 1997\end{array}$ \\
\hline 835 & $\begin{array}{l}37.983453 \\
12.065391 \\
\end{array}$ & $\begin{array}{l}\text { Sco. Camello } \\
\text { Marettimo Island }\end{array}$ & SLI & NTC & $5 \pm 0.5$ & $5 \pm 0.5$ & LUM (Cala Rossa) & $111 \pm 20$ & 5,2 & $\begin{array}{l}\text { Antonioli et al., } 2002 \\
\text { Mauz et al., } 1997\end{array}$ \\
\hline 839 & $\begin{array}{l}37.972651 \\
12.068992 \\
\end{array}$ & $\begin{array}{l}\text { Passo del Bue } \\
\text { Marettimo Island }\end{array}$ & SLI & MT & $5 \pm 1.5$ & $5 \pm 2$ & LUM (Cala Rossa) & $111 \pm 20$ & 4,3 & $\begin{array}{l}\text { Antonioli et al., } 2002 \\
\text { Mauz et al., } 1997\end{array}$ \\
\hline 854 & $\begin{array}{l}37.98835 \\
12.328824\end{array}$ & $\begin{array}{l}\text { Pietre Varate } \\
\text { Levanzo Island (Egadi } \\
\text { archipelago) }\end{array}$ & SLI & NTC & $5 \pm 0.5$ & $5 \pm 0.5$ & $\begin{array}{l}\text { STRAT ( } P \text {. latus or } \\
\text { Senegalese fauna) }\end{array}$ & MIS 5 & 5,2 & $\begin{array}{l}\text { Malatesta et al., } 1957 \\
\text { Ferranti et al., } 2006\end{array}$ \\
\hline 852 & $\begin{array}{l}37.987276 \\
12.340913\end{array}$ & $\begin{array}{l}\text { Cala Dogana } \\
\text { Levanzo Island (Egadi } \\
\text { archipelago) }\end{array}$ & SLI & MT & $5 \pm 1$ & $4.9 \pm 1.6$ & $\begin{array}{l}\text { STRAT ( } P \text {. latus or } \\
\text { Senegalese fauna) }\end{array}$ & MIS 5 & 4,3 & $\begin{array}{l}\text { Malatesta et al., } 1957 \\
\text { Ferranti et al., } 2006\end{array}$ \\
\hline 853 & $\begin{array}{l}37.989734 \\
12.345714 \\
\end{array}$ & $\begin{array}{l}\text { Cala Fredda } \\
\text { Levanzo Island (Egadi } \\
\text { archipelago) }\end{array}$ & SLI & MT & $5 \pm 1$ & $4.9 \pm 1.6$ & $\begin{array}{l}\text { STRAT ( } P . \text { latus or } \\
\text { Senegalese fauna) }\end{array}$ & MIS 5 & 4,3 & $\begin{array}{l}\text { Malatesta et al., } 1957 \\
\text { Ferranti et al., } 2006\end{array}$ \\
\hline 920 & $\begin{array}{l}38.033343 \\
12.526471 \\
\end{array}$ & $\begin{array}{l}\text { Trapani- Tonnara S. } \\
\text { Giuliano }\end{array}$ & SLI & BRK & $5 \pm 1$ & $5.3 \pm 1.3$ & $\begin{array}{l}\text { STRAT (P. latus or } \\
\text { Senegalese fauna) }\end{array}$ & MIS 5 & 3,3 & Ruggieri and Unti. 1974 \\
\hline 894 & $\begin{array}{l}38.101255 \\
12.658343 \\
\end{array}$ & $\begin{array}{l}\text { Monte Cofano } \\
\text { Trapani }\end{array}$ & SLI & MT & $20 \pm 2$ & $19.9 \pm 2.3$ & $\begin{array}{l}\text { STRAT ( } P \text {. latus or } \\
\text { Senegalese fauna) }\end{array}$ & MIS 5 & 4,3 & Antonioli et al., 2006c \\
\hline \multirow[b]{2}{*}{845} & \multirow{2}{*}{$\begin{array}{l}38.111868 \\
12.723722\end{array}$} & \multirow{2}{*}{$\begin{array}{l}\text { Castelluzzo } \\
\text { San Vito lo Capo }\end{array}$} & \multirow[b]{2}{*}{ SLI } & \multirow[b]{2}{*}{ MT } & \multirow[b]{2}{*}{$14 \pm 1.5$} & \multirow[b]{2}{*}{$14 \pm 2$} & LUM (Cala Rossa) & $111 \pm 20$ & \multirow[b]{2}{*}{4,4} & \multirow{2}{*}{$\begin{array}{l}\text { Antonioli et al., } 2002 \\
\text { Mauz et al., } 1997\end{array}$} \\
\hline & & & & & & & $\begin{array}{l}\text { STRAT (P. latus or } \\
\text { Senegalese fauna) }\end{array}$ & MIS 5 & & \\
\hline
\end{tabular}




\begin{tabular}{|c|c|c|c|c|c|c|c|c|c|c|}
\hline $\begin{array}{l}\text { WALIS } \\
\text { ID }\end{array}$ & $\begin{array}{l}\text { Latitude } \\
\text { Longitude }\end{array}$ & Site & $\begin{array}{l}\text { Type of } \\
\text { datapoint }\end{array}$ & $\begin{array}{l}\text { RSL } \\
\text { Indicator }\end{array}$ & $\begin{array}{l}\text { Elevation } \\
(\mathbf{m})\end{array}$ & $\begin{array}{l}\text { Paleo RSL } \\
\text { (m) }\end{array}$ & Dating (Original ID) & $\begin{array}{l}\text { Age (ka or } \\
\text { MIS) }\end{array}$ & $\begin{array}{l}\text { Quality } \\
\text { (RSL, } \\
\text { Age) }\end{array}$ & Reference(s) \\
\hline 830 & $\begin{array}{l}38.138155 \\
12.73782 \\
\end{array}$ & Macari & SLI & MT & $5 \pm 1$ & $4.9 \pm 1.6$ & $\begin{array}{l}\text { STRAT ( } P \text {. latus or } \\
\text { Senegalese fauna) }\end{array}$ & MIS 5 & 4,2 & Mauz et al., 1997 \\
\hline \multirow[b]{2}{*}{844} & \multirow{2}{*}{$\begin{array}{l}38.17592 \\
12.716799\end{array}$} & \multirow{2}{*}{$\begin{array}{l}\text { Cala Mancino } \\
\text { San Vito lo Capo }\end{array}$} & \multirow[b]{2}{*}{ SLI } & \multirow[b]{2}{*}{ NTC } & \multirow[b]{2}{*}{$7.1 \pm 0.5$} & \multirow[b]{2}{*}{$7.1 \pm 0.5$} & LUM (Cala Rossa) & $111 \pm 20$ & \multirow[b]{2}{*}{5,2} & \multirow{2}{*}{$\begin{array}{l}\text { Antonioli et al., } 2002 \\
\text { Mauz et al., } 1997\end{array}$} \\
\hline & & & & & & & $\begin{array}{l}\text { STRAT (P. latus or } \\
\text { Senegalese fauna) }\end{array}$ & MIS 5 & & \\
\hline \multirow[b]{2}{*}{923} & \multirow{2}{*}{$\begin{array}{l}38.181488 \\
12.719884\end{array}$} & \multirow{2}{*}{$\begin{array}{l}\text { San Vito lo Capo } \\
\text { W side }\end{array}$} & \multirow[b]{2}{*}{ SLI } & \multirow[b]{2}{*}{ MT } & \multirow[b]{2}{*}{$12.5 \pm 1.5$} & \multirow[b]{2}{*}{$12.4 \pm 1.9$} & U-series (ENEA1013) & $>19.7 \pm 5.3$ & \multirow[b]{2}{*}{3,4} & \multirow{2}{*}{$\begin{array}{l}\text { Abate et al., } 1991 \\
\text { Antonioli et al., } 2002\end{array}$} \\
\hline & & & & & & & $\begin{array}{l}\text { STRAT (P. latus or } \\
\text { Senegalese fauna) }\end{array}$ & MIS 5 & & \\
\hline \multirow[b]{2}{*}{922} & \multirow{2}{*}{$\begin{array}{l}38.185555 \\
12.733778\end{array}$} & \multirow{2}{*}{$\begin{array}{l}\text { San Vito lo Capo } \\
\text { NE side }\end{array}$} & \multirow[b]{2}{*}{ SLI } & \multirow[b]{2}{*}{ NTC } & \multirow[b]{2}{*}{$8 \pm 0.5$} & \multirow[b]{2}{*}{$8 \pm 0.5$} & U-series (ENEA1013) & $>19.7 \pm 5.3$ & \multirow[b]{2}{*}{5,2} & \multirow{2}{*}{$\begin{array}{l}\text { Abate et al., } 1991 \\
\text { Antonioli et al., } 2002\end{array}$} \\
\hline & & & & & & & $\begin{array}{l}\text { STRAT (P. latus or } \\
\text { Senegalese fauna) }\end{array}$ & MIS 5 & & \\
\hline \multirow[b]{2}{*}{828} & \multirow{2}{*}{$\begin{array}{l}38.056002 \\
12.846727\end{array}$} & \multirow[b]{2}{*}{ Cala Rossa } & \multirow[b]{2}{*}{ SLI } & & & & LUM (Cala Rossa) & $111 \pm 20$ & & \\
\hline & & & & MT & $5 \pm 1$ & $4.9 \pm 1.6$ & $\begin{array}{l}\text { STRAT (P. latus or } \\
\text { Senegalese fauna) }\end{array}$ & MIS 5 & 4,4 & Mauz et al., 1997 \\
\hline & & & & & & & LUM (Cala Bianca) & $140 \pm 24$ & & \\
\hline 829 & 12.850376 & Cala Bianca & SLI & MT & $5 \pm 1$ & $4.9 \pm 1.6$ & $\begin{array}{l}\text { STRAT (P. latus or } \\
\text { Senegalese fauna) }\end{array}$ & MIS 5 & 4,4 & Mauz et al., 1997 \\
\hline & & & & & & & LUM (San Cataldo) & $131 \pm 43$ & & \\
\hline 827 & 13.078016 & Torre San Cataldo & SLI & MT & $18 \pm 3.6$ & $17.9 \pm 3.8$ & $\begin{array}{l}\text { STRAT (P. latus or } \\
\text { Senegalese fauna) }\end{array}$ & MIS 5 & 2,4 & Mauz et al., 1997 \\
\hline 826 & $\begin{array}{l}38.08832 \\
13.078468 \\
\end{array}$ & Nocella & SLI & MT & $18 \pm 3.6$ & $17.9 \pm 3.8$ & LUM (Nocella) & $101 \pm 43$ & 2,3 & Mauz et al., 1997 \\
\hline & & & & & & & U-series (UST1002b) & $132 \pm 5$ & & Hearty, 1986b \\
\hline 916 & 38.705192 & Ustica & SLI & BRK & $30 \pm 6$ & $30.3 \pm 6.1$ & AAR (N/A) & $>121 \pm 36.3$ & 2,5 & Antonioli et al., 2006c \\
\hline & & & & & & & $\begin{array}{l}\text { STRAT ( } P \text {. latus or } \\
\text { Senegalese fauna) }\end{array}$ & MIS 5 & & de Vita et al., 1998 \\
\hline 897 & $\begin{array}{l}38.223639 \\
13.316765 \\
\end{array}$ & $\begin{array}{l}\text { Palermo } \\
\text { Capo Gallo }\end{array}$ & SLI & BRK & $2 \pm 0.4$ & $2.2 \pm 0.9$ & $\begin{array}{l}\text { STRAT ( } P \text {. latus or } \\
\text { Senegalese fauna) }\end{array}$ & MIS 5 & 5,3 & $\begin{array}{l}\text { Gignoux, } 1913 \\
\text { Antonioli et al., 2006c }\end{array}$ \\
\hline 832 & $\begin{array}{l}38.21937 \\
13.315839\end{array}$ & Capo Gallo & SLI & BRK & $2 \pm 0.4$ & $2.2 \pm 0.9$ & $\begin{array}{l}\text { STRAT ( } P . \text { latus or } \\
\text { Senegalese fauna) }\end{array}$ & MIS 5 & 4,2 & $\begin{array}{l}\text { Gignoux, } 1913 \\
\text { Benjamin et al., } 2017 \\
\text { Taviani, } 2014 \\
\text { Zazo et al., } 2013\end{array}$ \\
\hline 896 & $\begin{array}{l}38.147436 \\
13.368541 \\
\end{array}$ & Palermo & SLI & BRK & $10 \pm 2$ & $10.2 \pm 2.1$ & $\begin{array}{l}\text { STRAT ( } P \text {. latus or } \\
\text { Senegalese fauna) }\end{array}$ & MIS 5 & 4,3 & Fabiani, 1941 \\
\hline 831 & $\begin{array}{l}38.111247 \\
13.533749\end{array}$ & Capo Zafferano & SLI & NTC & $7 \pm 0.5$ & $7 \pm 0.5$ & U-series (N/A) & MIS5e & 5,2 & $\begin{array}{l}\text { Mauz et al., } 1997 \\
\text { Antonioli et al., 1994c } \\
\text { Antonioli et al., 1994a }\end{array}$ \\
\hline 863 & 38.035052 & Cefalù (II) & SII & NTC & $30+0.5$ & $30+05$ & AAR (Cefalù 1) & >MIS 5 & 52 & Antoniolict ol 2006 \\
\hline 005 & 14.039278 & La Kalura & $\mathrm{SL1}$ & 1010 & $50 \pm 0.0$ & $50 \pm 0.0$ & AAR (Cefalù 2) & $>$ MIS 5 & $J, 2$ & 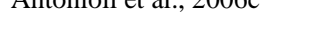 \\
\hline 862 & & Cefalù & SLI & NTC & $29 \pm 0.5$ & $29 \pm 0.5$ & AAR (Cefalù 1) & >MIS 5 & 5,2 & Antonioli et al., 2006c \\
\hline
\end{tabular}




\begin{tabular}{|c|c|c|c|c|c|c|c|c|c|c|}
\hline $\begin{array}{l}\text { WALIS } \\
\text { ID }\end{array}$ & $\begin{array}{l}\text { Latitude } \\
\text { Longitude }\end{array}$ & Site & $\begin{array}{l}\text { Type of } \\
\text { datapoint }\end{array}$ & $\begin{array}{l}\text { RSL } \\
\text { Indicator }\end{array}$ & $\begin{array}{l}\text { Elevation } \\
(\mathrm{m})\end{array}$ & $\begin{array}{l}\text { Paleo RSL } \\
\text { (m) }\end{array}$ & Dating (Original ID) & $\begin{array}{l}\text { Age (ka or } \\
\text { MIS) }\end{array}$ & $\begin{array}{l}\text { Quality } \\
\text { (RSL, } \\
\text { Age) }\end{array}$ & Reference(s) \\
\hline & $\begin{array}{l}38.040034 \\
14.02887\end{array}$ & & & & & & AAR (Cefalù 2) & $>$ MIS 5 & & \\
\hline 849 & $\begin{array}{l}37.500082 \\
15.067219\end{array}$ & Catania & SLI & MT & $165 \pm 16.5$ & $164.9 \pm 16.5$ & $\begin{array}{l}\text { OTHER (Clast of } \\
\text { Ancient Alkalic Centres } \\
\text { Unit) }\end{array}$ & $<$ MIS 7 & 2,2 & $\begin{array}{l}\text { Monaco et al., } 2002 \\
\text { Ferranti et al., } 2006 \\
\text { Gillot et al., } 1994\end{array}$ \\
\hline 850 & $\begin{array}{l}37.500082 \\
15.067219\end{array}$ & Catania (II) & SLI & MT & $100 \pm 10$ & $99.9 \pm 10$ & $\begin{array}{l}\text { OTHER (Clast of } \\
\text { Ancient Alkalic Centres } \\
\text { Unit) }\end{array}$ & $<$ MIS 7 & 2,2 & $\begin{array}{l}\text { Monaco et al., } 2002 \\
\text { Ferranti et al., } 2006 \\
\text { Gillot et al., } 1994 \\
\end{array}$ \\
\hline 851 & $\begin{array}{l}37.500082 \\
15.067219\end{array}$ & Catania (III) & SLI & MT & $100 \pm 10$ & $99.9 \pm 10$ & $\begin{array}{l}\text { OTHER (Clast of } \\
\text { Ancient Alkalic Centres } \\
\text { Unit) }\end{array}$ & $<$ MIS 7 & 2,2 & $\begin{array}{l}\text { Monaco et al., } 2002 \\
\text { Ferranti et al., } 2006 \\
\text { Gillot et al., } 1994\end{array}$ \\
\hline 848 & $\begin{array}{l}37.562777 \\
15.155716\end{array}$ & Aci Trezza (III) & SLI & MT & $100 \pm 10$ & $99.9 \pm 10$ & $\begin{array}{l}\text { OTHER (Clast of } \\
\text { Ancient Alkalic Centres } \\
\text { Unit) }\end{array}$ & $<$ MIS 7 & 2,3 & $\begin{array}{l}\text { Monaco et al., } 2002 \\
\text { Ferranti et al., } 2006 \\
\text { Gillot et al., } 1994\end{array}$ \\
\hline 847 & $\begin{array}{l}37.562777 \\
15.155716\end{array}$ & Aci Trezza (II) & SLI & MT & $125 \pm 12.5$ & $124.9 \pm 12.5$ & $\begin{array}{l}\text { OTHER (Clast of } \\
\text { Ancient Alkalic Centres } \\
\text { Unit) }\end{array}$ & MIS 7 & 2,3 & $\begin{array}{l}\text { Monaco et al., } 2002 \\
\text { Ferranti et al., } 2006 \\
\text { Gillot et al., } 1994\end{array}$ \\
\hline 846 & $\begin{array}{l}37.562777 \\
15.155716\end{array}$ & Aci Trezza & SLI & MT & $175 \pm 17.5$ & $174.9 \pm 17.5$ & $\begin{array}{l}\text { OTHER (Clast of } \\
\text { Ancient Alkalic Centres } \\
\text { Unit) }\end{array}$ & MIS 7 & 2,2 & $\begin{array}{l}\text { Monaco et al., } 2002 \\
\text { Ferranti et al., } 2006 \\
\text { Gillot et al., } 1994 \\
\end{array}$ \\
\hline \multirow{2}{*}{3626} & \multirow{2}{*}{$\begin{array}{l}37.853453 \\
15.296823\end{array}$} & \multirow{2}{*}{ Taormina (II) } & \multirow{2}{*}{ SLI } & \multirow{2}{*}{ NTC } & \multirow{2}{*}{$130 \pm 0.5$} & \multirow{2}{*}{$129.9 \pm 0.8$} & ESR (K4343) & $76.4 \pm 7$ & \multirow{2}{*}{5,2} & \multirow{2}{*}{$\begin{array}{l}\text { Bonfiglio, } 1981 \\
\text { Antonioli et al., 2006c } \\
\text { Catalano and De Guidi, } 2003\end{array}$} \\
\hline & & & & & & & ESR (k4244) & $103.3 \pm 12.5$ & & \\
\hline \multirow[b]{2}{*}{903} & \multirow{2}{*}{$\begin{array}{l}37.853453 \\
15.296823\end{array}$} & \multirow[b]{2}{*}{ Taormina } & \multirow[b]{2}{*}{ SLI } & \multirow[b]{2}{*}{ BRK } & \multirow[b]{2}{*}{$115 \pm 11.5$} & \multirow[b]{2}{*}{$114.9 \pm 11.5$} & ESR (K4343) & $76.4 \pm 7$ & \multirow[b]{2}{*}{2,3} & \multirow{2}{*}{$\begin{array}{l}\text { Antonioli et al., 2006b } \\
\text { Bonfiglio, 1981 } \\
\text { Catalano and De Guidi, } 2003 \\
\text { Antonioli et al., 2006c }\end{array}$} \\
\hline & & & & & & & ESR (k4244) & $103.3 \pm 12.5$ & & \\
\hline \multirow[b]{2}{*}{902} & \multirow{2}{*}{$\begin{array}{l}38.269623 \\
15.604196\end{array}$} & \multirow[b]{2}{*}{ Capo Peloro } & \multirow[b]{2}{*}{ SLI } & \multirow[b]{2}{*}{ MT } & \multirow[b]{2}{*}{$110 \pm 11$} & \multirow[b]{2}{*}{$109.9 \pm 11$} & AAR (20) & MIS 5e & \multirow[b]{2}{*}{2,4} & \multirow{2}{*}{$\begin{array}{l}\text { Bonfiglio \& Violanti, } 1983 \\
\text { Antonioli et al., 2004 } \\
\text { Hearty et al., 1986a } \\
\text { Hearty, 1986b }\end{array}$} \\
\hline & & & & & & & $\begin{array}{l}\text { STRAT (P. latus or } \\
\text { Senegalese fauna) }\end{array}$ & MIS 5 & & \\
\hline \multirow{2}{*}{921} & \multirow{2}{*}{$\begin{array}{l}38.255355 \\
15.246463\end{array}$} & \multirow{2}{*}{ Milazzo } & \multirow{2}{*}{ SLI } & \multirow{2}{*}{ MT } & \multirow{2}{*}{$90 \pm 9$} & \multirow{2}{*}{$89.9 \pm 9$} & $\operatorname{AAR}(21)$ & $>\operatorname{MIS} 5 \mathrm{c}$ & 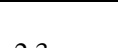 & Hearty et al., 1986a \\
\hline & & & & & & & $\operatorname{AAR}(21)$ & $>$ MIS 5c & 2,3 & Hearty, 1986b \\
\hline 918 & $\begin{array}{l}38.638881 \\
15.074178\end{array}$ & Panarea (II) & SLI & MT & $115 \pm 11.5$ & $115.2 \pm 11.5$ & AAR (23) & $>$ MIS $5 \mathrm{e}$ & 2,3 & $\begin{array}{l}\text { Lucchi et al., } 2004 \\
\text { Antonioli et al., 2006c } \\
\text { Hearty, 1986b }\end{array}$ \\
\hline 917 & $\begin{array}{l}38.638881 \\
15.074178\end{array}$ & Panarea & SLI & BRK & $50 \pm 10$ & $50.2 \pm 10$ & AAR (23) & $>$ MIS $5 \mathrm{e}$ & 2,3 & $\begin{array}{l}\text { Hearty, 1986b } \\
\text { Antonioli et al., 2006c }\end{array}$ \\
\hline 911 & $\begin{array}{l}38.479865 \\
14.900262\end{array}$ & Lipari (III) & SLI & MT & $12 \pm 1.2$ & $11.9 \pm 1.5$ & U-series (N/A) & $<119 \pm 6$ & 4,3 & $\begin{array}{l}\text { Lucchi et al., 2001 } \\
\text { Antonioli et al., 2006c } \\
\text { Calanchi et al., 2002 } \\
\text { Lucchi et al., 2004 } \\
\text { Lucchi et al., 2004b } \\
\text { Radke, 1986 }\end{array}$ \\
\hline
\end{tabular}




\begin{tabular}{|c|c|c|c|c|c|c|c|c|c|c|}
\hline $\begin{array}{l}\text { WALIS } \\
\text { ID }\end{array}$ & $\begin{array}{l}\text { Latitude } \\
\text { Longitude }\end{array}$ & Site & $\begin{array}{l}\text { Type of } \\
\text { datapoint }\end{array}$ & $\begin{array}{l}\text { RSL } \\
\text { Indicator }\end{array}$ & $\begin{array}{l}\text { Elevation } \\
\text { (m) }\end{array}$ & $\begin{array}{l}\text { Paleo RSL } \\
\text { (m) }\end{array}$ & Dating (Original ID) & $\begin{array}{l}\text { Age (ka or } \\
\text { MIS) }\end{array}$ & $\begin{array}{l}\text { Quality } \\
\text { (RSL, } \\
\text { Age) }\end{array}$ & Reference(s) \\
\hline 910 & $\begin{array}{l}38.479865 \\
14.900262\end{array}$ & Lipari (II) & SLI & MT & $25 \pm 2.5$ & $24.9 \pm 2.7$ & U-series (N/A) & $<119 \pm 6$ & 2,3 & $\begin{array}{l}\text { Lucchi et al., 2001 } \\
\text { Antonioli et al., 2006c } \\
\text { Calanchi et al., 2002 } \\
\text { Lucchi et al., 2004 } \\
\text { Lucchi et al., 2004b } \\
\text { Radke, 1986 }\end{array}$ \\
\hline 909 & $\begin{array}{l}38.479865 \\
14.900262\end{array}$ & Lipari (I) & SLI & MT & $44 \pm 4.4$ & $43.9 \pm 4.5$ & U-series (N/A) & $119 \pm 6$ & 2,3 & $\begin{array}{l}\text { Lucchi et al., 2001 } \\
\text { Antonioli et al., 2006c } \\
\text { Calanchi et al., 2002 } \\
\text { Lucchi et al., 2004 } \\
\text { Lucchi et al., 2004b } \\
\text { Radke, 1986 }\end{array}$ \\
\hline 914 & $\begin{array}{l}38.578052 \\
14.577761\end{array}$ & Filicudi & SLI & MT & $45 \pm 4.5$ & $45.2 \pm 4.6$ & ESR (N/A) & MIS 5e & 2,3 & $\begin{array}{l}\text { Lucchi et al., } 2004 \\
\text { Antonioli et al., 2006c } \\
\text { Radke, } 1986\end{array}$ \\
\hline 924 & $\begin{array}{l}38.075925 \\
14.65973 \\
\end{array}$ & Sant'Agata di Militello & SLI & MT & $100 \pm 10$ & $99.9 \pm 10$ & LUM (23) & $118 \pm 7$ & 2,3 & Giunta et al., 2012 \\
\hline 901 & $\begin{array}{l}37.268912 \\
15.224508\end{array}$ & $\begin{array}{l}\text { Augusta } \\
\text { Mt. Tauro }\end{array}$ & SLI & MT & $15 \pm 3$ & $14.9 \pm 3.1$ & $\begin{array}{l}\text { OTHER (Clast of } \\
\text { Ancient Alkalic Centres } \\
\text { Unit) }\end{array}$ & $<$ MIS 7 & 3,4 & $\begin{array}{l}\text { Di Grande \& Scamarda, } 1973 \\
\text { Antonioli et al., 2006c } \\
\text { Cosentino \& Gliozzi, } 1988 \\
\text { Di Grande \& Neri, } 1988 \\
\text { Gillot et al., } 1994 \\
\end{array}$ \\
\hline \multirow{8}{*}{3577} & \multirow{8}{*}{$\begin{array}{l}37.00389 \\
15.312381\end{array}$} & \multirow{8}{*}{ Plemmiro Cave } & \multirow{8}{*}{ TLI } & & \multirow{8}{*}{$-22.2 \pm 0.5$} & & U-series (JD073) & $75.3 \pm 0.6$ & \multirow{8}{*}{0,5} & \multirow{8}{*}{ Dutton et al., 2009} \\
\hline & & & & & & & U-series (JD074) & $72.4 \pm 0.8$ & & \\
\hline & & & & & & & U-series (JD076) & $72.7 \pm 1.5$ & & \\
\hline & & & & & & & U-series (JD077) & $74.2 \pm 1.1$ & & \\
\hline & & & & & & & U-series (JD082) & $75.2 \pm 1.4$ & & \\
\hline & & & & & & & U-series (JD083) & $74.7 \pm 0.9$ & & \\
\hline & & & & & & & U-series (JD084) & $76.5 \pm 1.3$ & & \\
\hline & & & & & & & U-series (JD085) & $73.7 \pm 0.8$ & & \\
\hline 900 & $\begin{array}{l}36.746989 \\
15.102658 \\
\end{array}$ & Pachino & SLI & BRK & $15 \pm 3$ & $15.2 \pm 3.1$ & $\begin{array}{l}\text { STRAT (P. latus or } \\
\text { Senegalese fauna) }\end{array}$ & MIS 5 & 3,3 & Malatesta, 1985 \\
\hline 907 & $\begin{array}{l}35.506086 \\
12.605981 \\
\end{array}$ & Lampedusa & SLI & BRK & $4 \pm 0.8$ & $4.4 \pm 1.3$ & $\begin{array}{l}\text { STRAT ( } P \text {. latus or } \\
\text { Senegalese fauna) }\end{array}$ & MIS 5 & 4,3 & $\begin{array}{l}\text { Segre, } 1960 \\
\text { Buccheri et al., } 1999\end{array}$ \\
\hline \multicolumn{11}{|l|}{ Algeria } \\
\hline 4 & $\begin{array}{l}36.63235 \\
2.40189\end{array}$ & $\begin{array}{l}\text { Chenoua } \\
\text { Transect AT1 }\end{array}$ & SLI & MT & $3.5 \pm 1$ & $3.5 \pm 1.5$ & $\begin{array}{l}\text { STRAT (Tipaza } \\
\text { Eemian Terrace) }\end{array}$ & MIS 5 & 3,2 & $\begin{array}{l}\text { Authemayou et al., } 2017 \\
\text { Saoudi, } 1989 \\
\text { Stearns and Thurber, } 1965\end{array}$ \\
\hline 5 & $\begin{array}{l}36.591389 \\
2.450042\end{array}$ & $\begin{array}{l}\text { Tipasa } \\
\text { Transect AT2 }\end{array}$ & SLI & MT & $8 \pm 3$ & $8 \pm 3.2$ & $\begin{array}{l}\text { STRAT (Tipaza } \\
\text { Eemian Terrace) }\end{array}$ & MIS 5 & 2,2 & $\begin{array}{l}\text { Authemayou et al., } 2017 \\
\text { Saoudi, } 1989 \\
\text { Stearns and Thurber, } 1965\end{array}$ \\
\hline
\end{tabular}




\begin{tabular}{|c|c|c|c|c|c|c|c|c|c|c|}
\hline $\begin{array}{l}\text { WALIS } \\
\text { ID }\end{array}$ & $\begin{array}{l}\text { Latitude } \\
\text { Longitude }\end{array}$ & Site & $\begin{array}{l}\text { Type of } \\
\text { datapoint }\end{array}$ & $\begin{array}{l}\text { RSL } \\
\text { Indicator }\end{array}$ & $\begin{array}{l}\text { Elevation } \\
(\mathbf{m})\end{array}$ & $\begin{array}{l}\text { Paleo RSL } \\
\text { (m) }\end{array}$ & Dating (Original ID) & $\begin{array}{l}\text { Age (ka or } \\
\text { MIS) }\end{array}$ & $\begin{array}{l}\text { Quality } \\
\text { (RSL, } \\
\text { Age) }\end{array}$ & Reference(s) \\
\hline \multirow[b]{2}{*}{6} & \multirow{2}{*}{$\begin{array}{l}36.617189 \\
2.644633\end{array}$} & \multirow{2}{*}{$\begin{array}{l}\text { Bou Haroun } \\
\text { Transect AT3 }\end{array}$} & \multirow[b]{2}{*}{ SLI } & \multirow[b]{2}{*}{ MT } & \multirow[b]{2}{*}{$7 \pm 1$} & \multirow[b]{2}{*}{$7 \pm 1.5$} & U-series (21) & MIS 5 & \multirow[b]{2}{*}{2,2} & \multirow{2}{*}{$\begin{array}{l}\text { Authemayou et al., } 2017 \\
\text { Saoudi, } 1989 \\
\text { Stearns and Thurber, } 1965\end{array}$} \\
\hline & & & & & & & $\begin{array}{l}\text { STRAT (Tipaza } \\
\text { Eemian Terrace) }\end{array}$ & MIS 5 & & \\
\hline 7 & $\begin{array}{l}36.594578 \\
2.572592\end{array}$ & $\begin{array}{l}\text { Tipasa } \\
\text { Transect AT2' }\end{array}$ & SLI & MT & $6 \pm 2$ & $6 \pm 2.3$ & $\begin{array}{l}\text { STRAT (Tipaza } \\
\text { Eemian Terrace) }\end{array}$ & MIS 5 & 2,1 & $\begin{array}{l}\text { Authemayou et al., } 2017 \\
\text { Saoudi, } 1989 \\
\text { Stearns and Thurber, } 1965\end{array}$ \\
\hline 8 & $\begin{array}{l}36.653492 \\
2.77926\end{array}$ & $\begin{array}{l}\text { Mazafran } \\
\text { Transect AT4 }\end{array}$ & SLI & MT & $6 \pm 1$ & $6 \pm 1.5$ & $\begin{array}{l}\text { STRAT (Tipaza } \\
\text { Eemian Terrace) }\end{array}$ & MIS 5 & 2,1 & $\begin{array}{l}\text { Authemayou et al., } 2017 \\
\text { Saoudi, } 1989 \\
\text { Stearns and Thurber, } 1965\end{array}$ \\
\hline \multirow[b]{2}{*}{9} & \multirow{2}{*}{$\begin{array}{l}36.798089 \\
2.902117\end{array}$} & \multirow{2}{*}{$\begin{array}{l}\text { Ain Benian } \\
\text { Transect AT5 }\end{array}$} & \multirow[b]{2}{*}{ SLI } & \multirow[b]{2}{*}{ MT } & \multirow[b]{2}{*}{$8 \pm 3$} & \multirow[b]{2}{*}{$8 \pm 3.2$} & U-series (48) & MIS 5e & \multirow[b]{2}{*}{2,1} & \multirow{2}{*}{$\begin{array}{l}\text { Authemayou et al., } 2017 \\
\text { Saoudi, } 1989 \\
\text { Stearns and Thurber, } 1965\end{array}$} \\
\hline & & & & & & & $\begin{array}{l}\text { STRAT (Tipaza } \\
\text { Eemian Terrace) }\end{array}$ & MIS 5 & & \\
\hline 11 & $\begin{array}{l}35.866385 \\
-0.298541 \\
\end{array}$ & Arzew & SLI & MT & $38 \pm 10$ & $38 \pm 10.1$ & $\begin{array}{l}\text { STRAT ( } P . \text { latus or } \\
\text { Senegalese fauna) }\end{array}$ & MIS 5 & 1,1 & Meghraoui et al., 1996 \\
\hline 12 & $\begin{array}{l}35.574939 \\
-1.180597 \\
\end{array}$ & Cap Figalo & SLI & MT & $45 \pm 10$ & $45 \pm 10.1$ & $\begin{array}{l}\text { STRAT (P. latus or } \\
\text { Senegalese fauna) }\end{array}$ & MIS 5 & 1,1 & Meghraoui et al., 1996 \\
\hline \multicolumn{11}{|l|}{ Morocco } \\
\hline \multirow{4}{*}{3599} & \multirow{4}{*}{$\begin{array}{l}35.828994 \\
-5.715446\end{array}$} & \multirow{4}{*}{$\begin{array}{l}\text { Hejar Lasfar marine } \\
\text { terrace }\end{array}$} & \multirow{4}{*}{ SLI } & \multirow{4}{*}{ MT } & \multirow{4}{*}{$13.5 \pm 2.7$} & \multirow{4}{*}{$13 \pm 3.4$} & U-series (S2-DC) & $106.8 \pm 3.3$ & \multirow{4}{*}{3,4} & \multirow{4}{*}{ El Abdellaoui et al., 2016} \\
\hline & & & & & & & U-series (S2-FC) & $96.6 \pm 2.3$ & & \\
\hline & & & & & & & U-series (S3-C1) & $111.2 \pm 2$ & & \\
\hline & & & & & & & U-series (S3-C2) & $119.6 \pm 2.3$ & & \\
\hline \multirow{2}{*}{248} & \multirow{2}{*}{$\begin{array}{l}35.83079 \\
-5.64211\end{array}$} & Dhada marine terrace & SUI & MT & $14+28$ & $134+35$ & U-series (S2-DC) & $106.8 \pm 3.3$ & 31 & Fl Abdellaomi et al 2016 \\
\hline & & $\mathrm{S} 2$ & $\mathrm{NL1}$ & 1010 & $1+12.0$ & $10.4 \pm .0$ & U-series (S2-FC) & $96.6 \pm 2.3$ & $J, 1$ & El Aoderraoul et al., 2010 \\
\hline 249 & 35.8312 & Dhada marine terrace & SUI & MT & $14+28$ & $134+35$ & U-series (S3-C1) & $111.2 \pm 2$ & & \\
\hline 249 & -5.641645 & $\mathrm{~S} 3$ & SLI & M1 & $14 \pm 2.8$ & $13.4 \pm 3.5$ & U-series (S3-C2) & $119.6 \pm 2.3$ & 3,5 & El Abdellaouı et al., 2016 \\
\hline & & & & & & & U-series (S2-DC) & $106.8 \pm 3.3$ & & \\
\hline 3508 & 35.868426 & Zhâra marine terrace & SII & MT & $135+27$ & $13+34$ & U-series (S2-FC) & $96.6 \pm 2.3$ & 34 & Fl Abdellooui et al 2016 \\
\hline 3598 & -5.511586 & Znara marine terrace & SLI & MI & $13.5 \pm 2.1$ & $13 \pm 3.4$ & U-series (S3-C1) & $111.2 \pm 2$ & 3,4 & El Abdellaoui et al., 2010 \\
\hline & & & & & & & U-series (S3-C2) & $119.6 \pm 2.3$ & & \\
\hline 260 & $\begin{array}{l}35.910136 \\
-5.414401 \\
\end{array}$ & $\begin{array}{l}\text { Cape Leona } \\
\text { Section C }\end{array}$ & SLI & NTC & $10.5 \pm 2.3$ & $10.5 \pm 2.3$ & U-series (MABE1005) & $84.4 \pm 3.3$ & 3,3 & Abad et al., 2013 \\
\hline 259 & 35.910333 & Bel Younech Beach & MLI & & $143+33$ & & U-series (MABE0604) & $131.2 \pm 7.4$ & & \\
\hline 239 & -5.385481 & Section A & VILI & & $14.3 \pm 5.3$ & & U-series (MABE0603) & $87 \pm 3.7$ & 0,1 & Abad et al., 2013 \\
\hline 4073 & & & & & & & U-series (MA-16) & $130 \pm 39$ & & \\
\hline $40 / 3$ & -5.215318 & Laskrine & SLI & M1 & $0 \pm 1.2$ & $5.8 \pm 1.5$ & LUM (MA-17) & $128.3 \pm 19.2$ & 2,4 & Bruckner, 1986 \\
\hline
\end{tabular}




\begin{tabular}{|c|c|c|c|c|c|c|c|c|c|c|}
\hline $\begin{array}{l}\text { WALIS } \\
\text { ID }\end{array}$ & $\begin{array}{l}\text { Latitude } \\
\text { Longitude }\end{array}$ & Site & $\begin{array}{l}\text { Type of } \\
\text { datapoint }\end{array}$ & $\begin{array}{l}\text { RSL } \\
\text { Indicator }\end{array}$ & $\begin{array}{l}\text { Elevation } \\
\text { (m) }\end{array}$ & $\begin{array}{l}\text { Paleo RSL } \\
\text { (m) }\end{array}$ & Dating (Original ID) & $\begin{array}{l}\text { Age (ka or } \\
\text { MIS) }\end{array}$ & $\begin{array}{l}\text { Quality } \\
\text { (RSL, } \\
\text { Age) }\end{array}$ & Reference(s) \\
\hline \multirow{2}{*}{4074} & \multirow{2}{*}{$\begin{array}{l}35.514598 \\
-5.159386\end{array}$} & \multirow{2}{*}{ Aouchtame } & \multirow{2}{*}{ SLI } & \multirow{2}{*}{ MT } & \multirow{2}{*}{$4 \pm 0.8$} & \multirow{2}{*}{$3.8 \pm 1.2$} & U-series (MA-13) & $87-100$ & \multirow{2}{*}{2,3} & \multirow{2}{*}{ Brückner, 1986} \\
\hline & & & & & & & U-series (MA-13b) & $73-82$ & & \\
\hline \multirow[b]{2}{*}{929} & \multirow{2}{*}{$\begin{array}{l}35.255483 \\
-3.92068\end{array}$} & \multirow[b]{2}{*}{ Al Hoceima } & \multirow[b]{2}{*}{ MLI } & & \multirow[b]{2}{*}{$2 \pm 0.4$} & & AAR (46) & MIS 5e & \multirow[b]{2}{*}{0,4} & \multirow[b]{2}{*}{ Hearty, 1986b } \\
\hline & & & & & & & $\begin{array}{l}\text { STRAT (P. latus or } \\
\text { Senegalese fauna) }\end{array}$ & MIS 5 & & \\
\hline \multirow{4}{*}{3622} & \multirow{4}{*}{$\begin{array}{l}35.281955 \\
-3.706314\end{array}$} & \multirow{4}{*}{$\begin{array}{l}\text { Rastarf Cape } \\
\text { Al-Hoceima }\end{array}$} & \multirow{4}{*}{ SLI } & \multirow{4}{*}{ MT } & \multirow{4}{*}{$22 \pm 1$} & \multirow{4}{*}{$22 \pm 1.5$} & U-series (Co1) & $<213 \pm 63.9$ & \multirow{4}{*}{3,2} & \multirow{4}{*}{ Poujol et al., 2014} \\
\hline & & & & & & & U-series (Co2) & $<207 \pm 62.1$ & & \\
\hline & & & & & & & U-series (S1) & $\begin{array}{l}<203+34 /- \\
27\end{array}$ & & \\
\hline & & & & & & & U-series (S2) & $\begin{array}{l}<186+20 /- \\
18\end{array}$ & & \\
\hline 4075 & $\begin{array}{l}35.204607 \\
-3.30254 \\
\end{array}$ & Beni Said & SLI & MT & $5 \pm 1$ & $5 \pm 1.5$ & $\begin{array}{l}\text { STRAT ( } P \text {. latus or } \\
\text { Senegalese fauna) }\end{array}$ & MIS 5 & 2,3 & Brückner, 1986 \\
\hline \multirow{4}{*}{4076} & \multirow{4}{*}{$\begin{array}{l}35.227333 \\
-3.211035\end{array}$} & \multirow{4}{*}{ Ifri Ifounassene } & \multirow{4}{*}{ SLI } & \multirow{4}{*}{ MT } & \multirow{4}{*}{$5 \pm 1$} & \multirow{4}{*}{$5 \pm 1.5$} & U-series (MA-1b) & $72-83$ & \multirow{4}{*}{2,4} & \multirow{4}{*}{ Brückner, 1986} \\
\hline & & & & & & & ESR (MA1a) & $94.3 \pm 18.9$ & & \\
\hline & & & & & & & ESR (MA1b) & $133.9 \pm 26.8$ & & \\
\hline & & & & & & & $\begin{array}{l}\text { STRAT (P. latus or } \\
\text { Senegalese fauna) }\end{array}$ & MIS 5 & & \\
\hline 4077 & 35.226628 & Ifri Ifoungsene (II) & SII & MT & $38+08$ & $3+14$ & U-series (MA-2a) & $65-81$ & 3 & Priïlner 1086 \\
\hline 4011 & -3.209835 & 1111 Hounassene (II) & SL1 & N11 & $3.0 \pm 0.0$ & $3.1 \pm 1.4$ & U-series (MA-2b) & $62-70$ & 2,0 & Dтискпет, 1900 \\
\hline & & & & & & & U-series (MA-3b) & $93-118$ & & \\
\hline 4078 & $\begin{array}{l}35.225917 \\
-3.209594\end{array}$ & Ifri Ifounassene (III) & SLI & MT & $5 \pm 1$ & $5 \pm 1.5$ & ESR (MA-3a) & $140.4 \pm 28.1$ & 2,4 & Brückner, 1986 \\
\hline & & & & & & & ESR (MA-3b) & $114.8 \pm 23$ & & \\
\hline
\end{tabular}

